\title{
Laboratory Directed Research and Development Program FY 2009
}

March 2010 


\section{DISCLAIMER}

This document was prepared as an account of work sponsored by the United States Government. While this document is believed to contain correct information, neither the United States Government nor any agency thereof, nor The Regents of the University of California, nor any of their employees, makes any warranty, express or implied, or assumes any legal responsibility for the accuracy, completeness, or usefulness of any information, apparatus, product, or process disclosed, or represents that its use would not infringe privately owned rights. Reference herein to any specific commercial product, process, or service by its trade name, trademark, manufacturer, or otherwise, does not necessarily constitute or imply its endorsement, recommendation, or favoring by the United States Government or any agency thereof, or The Regents of the University of California. The views and opinions of authors expressed herein do not necessarily state or reflect those of the United States Government or any agency thereof or The Regents of the University of California.

Lawrence Berkeley Laboratory is an equal opportunity employer. 


\section{Report on \\ Ernest Orlando Lawrence Berkeley National Laboratory}

\section{Laboratory Directed Research and Development Program}

FY 2009

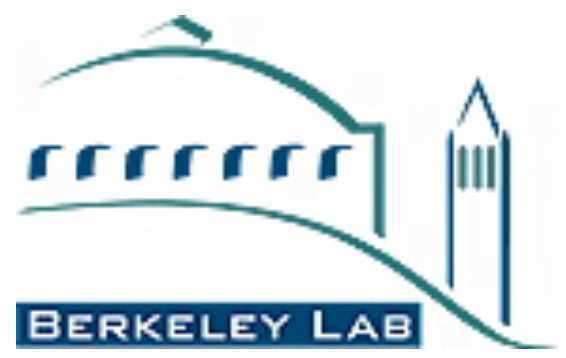

Ernest Orlando Lawrence Berkeley National Laboratory Berkeley, CA 94720

MARCH, 2010

Prepared for the U.S. Department of Energy under Contract No. DE-AC02-05CH11231 



\section{Table of Contents}

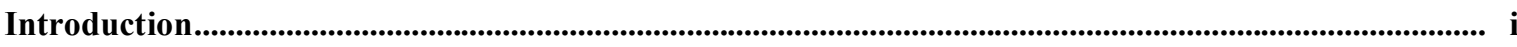

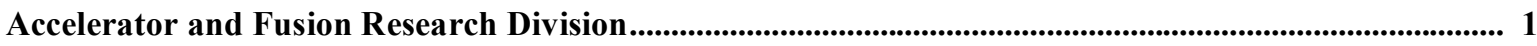

Fernando Sannibale $\quad$ Experimental Accelerator R\&D Toward a Future Light Source .... 1

John Corlett

John Staples

Russell Wells

Alexander Zholents

Thomas Schenkel

Quantum Information Science with Integrated Color Centers in Diamond

Csaba Toth

Experimental Demonstration of a Laser-Plasma-Accelerator

Carl Schroeder Driven Free-Electron Laser

William Fawley

Kem Robinson

Jean-Luc Vay

William Fawley

Lorentz Compaction of Scales for Ultra-efficient Simulation of Advance Accelerators (and other systems) ................................... 4

Marco Venturini

Emittance Manipulation and Beam Conditioning for FEL's.

et al

Weishi Wan

High Brightness Photocathodes as Electron Sources for FELs ......

Emanuele Pedersoli

C. Michael Greaves

Howard Padmore

William Fawley

Gregory Penn

Alexander Zholents

FEL Concepts for Multiple Independent X-ray Beamlines ........

Advanced Light Source Division

Elke Arenholz

Soren Prestemon

YiDe Chuang

Jinghua Guo

Alexander Hexemer et al

Howard Padmore

Eli Rotenberg

Aaron Bostwick

David Shapiro

Yiqiong Zhao

Valeriy Yashchuk

Kenneth Goldberg Valeriy Yashchuk
Optimization of Flux Pinning in Type II Superconductor Based

Magnets for Soft X-ray Scattering Applications

Building In-situ Electronic Structure Study Capability with Photon-in/Photon-out Soft X-ray Spectroscopy

Soft X-Ray Scattering as a New Probe of Polymer Systems

High Quantum Yield Multi-Alkali Cathodes for psec Pulsed Electron Sources

Improved Electron Detection System for Extreme AngleResolved Photoemission Experiments.

Development of a Laser Goniometer for X-ray and Electron Diffraction Microscopy

Ultra-high Resolution Optics for Soft X-ray Inelastic Scattering...

X-Ray Optical Metrology for Coherence-Preserving Adaptive Optics.... 
Hendrik Bluhm

Kevin Wilson

Oliver Gessner

Mary Gilles

Charles Harris

F. Dean Toste
Chemical Reactions at Liquid/Vapor Interfaces Probed by Photoemission Spectroscopy.

Probing Transient Molecular Entanglement Using Femtosecond High Resolution Delayed-Field Coincidence Imaging

Development of In Situ Cells for Reactive Spectroscopic and Microscopic Studies

Dynamics of Homogeneous Catalysis Reactions Investigated with Transient Two-Dimensional Infrared Spectroscopy .

Metal Nitrosyl Complexes and Catalytic C-H Bond

Functionalization

\section{Computing Sciences}

(National Energy Research Scientific Computing Center, and Computational Research Divisions) .. 21
Structured, Adaptive Mesh Refinement Method for Multiphase

Reactive Transport in Groundwater

Low Order Models for Simulation of Ballistic Charge Transport in Nanoscale Devices

Enhancing the Effectiveness of Manycore Chip Technologies for High-End Computing

On-Demand Overlays for Scientific Applications.....

Energy Smart Disk-Based Mass Storage System 25

Holistic Approach to Energy Efficient Computing Architecture.... 26

Reference Benchmarks for the Dwarfs (Algorithms)

Managing Petascale Data with Emerging Computer

Architectures

Earth Sciences Division

Application of Adjoint Field Methods and Time-Reversal Data Processing to Inverse Problems in Electromagnetics, Seismics, and Ultrasonics.

Eoin Brodie

Uncoverinig the Mechanistic Basis for Soil Microbial

Community Response to Altered Precipitation Patterns

William Collins

Integrated Earth Systems Climate Modeling and Analysis

Maximizing Photosynthetic Yield by Increasing Sink Strength.....

Microbiomics of Complex Microbial Communities in Environmental Samples.

Hui-Hai Liu Impact of Climate Change on Soil Water Dynamics in Arid Areas. 
Rohit Salve

Identifying and Predicting Climate Change Impacts on the

William Dietrich

Land-Based Components of the Water Cycle.

Stefan Finsterle

Environmental Energy Technologies Division

Vladimir Bazjanac

Hugo Destaillats

Phil Haves

Alberto Vincentelli

John Kerr

Venkat Srinivasan

Marca Deoff

Mark Levine

David Lorenzetti

Michael Sohn

Melissa Lunden

Randy Maddalena Thomas McKone

Michael Sohn

Mark Mendell

Douglas Black

Philip Price

Francis Rubinstein

Alan Sanstad

Michael Sohn

Michael Wetter
Embedded Engineering, Construction Materials, and HVAC Components in Modular Energy Systems Simulation 36

Understanding the Chemistry of Innovative Air Cleaning Technologies 37

Development of a Building Operating Platform 38

Development of Novel Improved Capacitors for Pulse Power Applications

China's Energy Future: Changes in Energy Intensity

Probabilistic Optimization of Energy Systems in Buildings

Novel Techniques to Characterize Secondary Organic Aerosols

Formed From Gas-phase Volatile Organic Compounds Emitted

From Biogenic Sources

Relating Tissue Residues to Indoor Chemical Sources in a Bayesian Framework: Synthesis of Indoor Chemistry, Pharmacokinetics, and Biomarkers

Using New Microbial Assays to Characterize Dampness-related Exposures: Combining Molecular Biology with Building, Aerosol, and Health Sciences

Self-Tuning Building Energy Model

Using Internet Protocols (IP) Telephony and Wireless

Technologies to Extend the Reach of Conventional Building

Automation Systems.

Inverse Approach to Characterizing Uncertain Economic Drivers of Global Climate Change.....

Software for Integrated Analysis of Sensor Data for Advanced Energy Controls

Building Informatics Environment Enabling Rapid Prototyping and Model Extraction for Building Automation Systems.

Genomics Division

Mark Biggin

Jian Jin

Len Pennacchio

Axel Visel
Measurement of Protein/DNA Binding in vitro and in vivo

Using Single Molecule Approaches

Assessing Epigenomic Approaches for Gene Enhancer

Discovery...... 
Gareth Butland

Luis Comolli

Bo Hang

Terumi Kohwi-

Shigematsu et al

Cynthia McMurray

John Tainer

Andrew Wyrobek

Francesco Marchetti

Functional Interactomics: Integrating Physical and Functional Interaction Networks

Linking Genomics, Proteomics and Ultrastructural Characterization of Microbial Communities and Their Viruses.....

Interaction of Fragile X Mental Retardation Protein with Thymine-DNA Glycosylase: Implication in the Molecular Mechanism of Fragile X Syndrome.

Genome Organizer in Carcinogenesis ..... 55

Fluorescence Energy Transfer (FRET) Methodology for Visualization of Simultaneous and Reversible Interactions (SPARKLE)

Bio Energy Technologies and Science Integrated Efficiently (BETSIE).

Expression Profiling of Radiation and Cancer Susceptibility Genes . 58

Materials Sciences Division.

Nitash Balsara
Blandine Jérôme
Jeffrey Long
Delia Milliron

Andrew Minor

Robert Schoenlein

Irfan Siddiqi

Berend Smit

Ting $\mathrm{Xu}$

Jeffrey Urban

et al

Junqiao $\mathrm{Wu}$
Synthesis and Characterization of Self-Assembled Battery

Electrodes

Transport in Thin Polymer Films. 60

Conducting Metal-Organic Frameworks

Solution-Processed Inorganic Composites with Mixed Transport Characteristics....

In Situ Electromagnetic Probing in a Transmission Electron Microscope (TEM)

X-ray Studies of Charge-Order Dynamics in Complex Materials.. 64

Quantifying the Quantum Backaction of a Non-Linear Dispersive Measurement 65

Self-assembly of Membrane Proteins 66

Understanding How Nanoscale Interfaces Modify Predicted Optical, Vibrational, and Electronic Properties

Microscopic Mechanism of Resistance Switching Memory Effect..

Nuclear Science Division

Shamsuzzoha Basunia Richard Firestone Roderick Clark

Spencer Klein

Development of a $100 \mathrm{~km}^{3}$ Neutrino Detector for Ultra High Energy Neutrinos

Kevin Lesko 
Kai Vetter Ultra-sensitive Ge Detectors for Low-background Physics

Experiments..................................................................... 72

Feng Yuan $\quad$ Theoretical Study of Nucleon Structure ........................................ 73

Bowen Xiao

Physical Biosciences Division.

Paul Adams

Peter Zwart

Alexander Hexemer

Carlos Bustamante

Michelle Chang

Gavin Crooks

Daniel Fletcher

Jay Groves

Jan Liphardt

Manfred Auer

Ahmet Yildiz
Development of Reusable Software Modules for the Analyses of bioSAXS Data

Structural and Functional Characterization of DNA

Translocation Across Membranes by SpoIIIE Using Advanced

Microcopies

Biological Methods for Synthesis of Iron-based Nanomaterials ... 76

Understanding the Nanothermodynamics of Molecular

Machines.

Engineering Environmental Sensitivity in an Artificial Cell 78

Applications of Hybrid Live Cell - Synthetic Devices for Cancer Research.

Light-boosted Fermentation in the Yeast Saccharomyces cerevisiae. 80

Control of Intraflagellar Transport in Chlamydomonas Cells 81

\section{Physics Division}

Marco Battaglia

Peter Denes

Christian Bauer

Maurice Garcia-Sciveres

Murdock Gilchriese

Carl Haber

Nikhil Padmanabhan

Martin White

David Schlegel

Natalie Roe

Peter Denes

Stephen Holland

David Schlegel

George Smoot

Alexie Leauthaud
Advanced Silicon Detectors for Future Short Pulse X-ray

Sources.

Soft Collinear Effective Theories Applied to Collider Physics ...... 83

Development of Nanowire Carpet Hybrid Pixel (NCHyP)

Detectors.....

Development of Multi-Modular Assemblies with Reduced Material and Services for Tracking at Future Colliders

Calibrating Baryon Acoustic Oscillations for Future Dark

Energy Experiments.

R\&D for Fast, Low-noise CCD Readout and Single Photon

Detection Capability

Baryon Oscillations and Dark Energy: Protyping Instruments. 88

Decoding Dark Energy with Weak Gravitational Lensing .... 89 


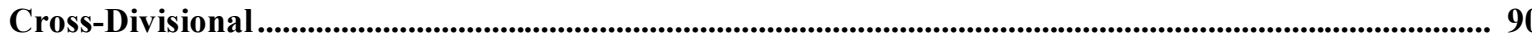

Manfred Auer

Integrated Tools in Multiscale Imaging .....

Jan Liphardt

Phil Hugenholtz

Terumi Kohwi-

Shigematsu

Seung-Wuk Lee

Fabrication of Photovoltaic Devices Using Nano-structured

Biomaterials

Lee Schroeder

Coupled Process Models, Separations, and Monitoring for

et al

Advanced Nuclear Fuel Cycles

92

Publications List 


\section{Introduction}

The Ernest Orlando Lawrence Berkeley National Laboratory (Berkeley Lab or LBNL) is a multi-program national research facility operated by the University of California for the Department of Energy (DOE). As an integral element of DOE's National Laboratory System, Berkeley Lab supports DOE's missions in fundamental science, energy resources, and environmental quality. Berkeley Lab programs advance four distinct goals for $\mathrm{DOE}$ and the nation:

- To perform leading multidisciplinary research in the computing sciences, physical sciences, energy sciences, biosciences, and general sciences in a manner that ensures employee and public safety and protection of the environment.

- To develop and operate unique national experimental facilities for qualified investigators.

- To educate and train future generations of scientists and engineers to promote national science and education goals.

- To transfer knowledge and technological innovations and to foster productive relationships among Berkeley Lab's research programs, universities, and industry in order to promote national economic competitiveness.

Berkeley Lab's research and the Laboratory Directed Research and Development (LDRD) program support DOE's Strategic Themes that are codified in DOE's 2006 Strategic Plan (DOE/CF-0010), with a primary focus on Scientific Discovery and Innovation. For that strategic theme, the Fiscal Year (FY) 2009 LDRD projects support each one of the three goals through multiple strategies described in the plan. In addition, LDRD efforts support the four goals of Energy Security, the two goals of Environmental Responsibility, and Nuclear Security (unclassified fundamental research that supports stockpile safety and nonproliferation programs). The LDRD program supports Office of Science strategic plans, including the 20year Scientific Facilities Plan and the Office of Science Strategic Plan. The research also supports the strategic directions periodically under consideration and review by the Office of Science Program Offices, such as LDRD projects germane to new research facility concepts and new fundamental science directions.

Berkeley Lab LDRD program also play an important role in leveraging DOE capabilities for national needs. The fundamental scientific research and development conducted in the program advances the skills and technologies of importance to our Work For Others (WFO) sponsors. Among many directions, these include a broad range of health-related science and technology of interest to the National Institutes of Health, breast cancer and accelerator research supported by the Department of Defense, detector technologies that should be useful to the Department of Homeland Security, and particle detection that will be valuable to the Environmental Protection Agency.

The Berkeley Lab Laboratory Directed Research and Development Program FY2009 report is compiled from annual reports submitted by principal investigators following the close of the fiscal year. This report describes the supported projects and summarizes their accomplishments. It constitutes a part of the LDRD program planning and documentation process that includes an annual planning cycle, project selection, implementation, and review.

The Berkeley Lab LDRD program is a critical tool for directing the Laboratory's forefront scientific research capabilities toward vital, excellent, and emerging scientific challenges. The program provides the resources for Berkeley Lab scientists to make rapid and significant contributions to critical national science and technology problems. The LDRD program also advances Berkeley Lab's core competencies, foundations, and scientific capability, and permits exploration of exciting new opportunities. All projects are work in forefront areas of science and technology. Areas eligible for support include the following:

- Advanced study of hypotheses, concepts, or innovative approaches to scientific or technical problems;

- Experiments and analyses directed toward "proof of principle" or early determination of the utility of new scientific ideas, technical concepts, or devices; and

- Conception and preliminary technical analyses of experimental facilities or devices.

The LDRD program supports Berkeley Lab's mission in many ways. First, because LDRD funds can be allocated within a relatively short time frame, Berkeley Lab researchers can support the mission of the Department of Energy (DOE) and serve the needs of the nation by quickly responding to forefront scientific problems. Second, LDRD enables Berkeley Lab to attract and retain highly qualified scientists and to support their efforts to carry out worldleading research. In addition, the LDRD program also supports new projects that involve graduate students and postdoctoral fellows, thus contributing to the education mission of Berkeley Lab.

Berkeley Lab has a formal process for allocating funds for the LDRD program. The process relies on individual 
scientific investigators and the scientific leadership of Berkeley Lab to identify opportunities that will contribute to scientific and institutional goals. The process is also designed to maintain compliance with DOE Orders, in particular DOE Order 413.2B (dated April 19, 2006). From year to year, the distribution of funds among the scientific program areas changes. This flexibility optimizes Berkeley Lab's ability to respond to opportunities.

Berkeley Lab LDRD policy and program decisions are the responsibility of the Laboratory Director. The Director has assigned general programmatic oversight responsibility to the Deputy Director, with administration and reporting on the LDRD program supported by that office. LDRD accounting procedures and financial management are consistent with the Laboratory's accounting principles and stipulations under the contract between the University of California and the Department of Energy, with accounting maintained through the Laboratory's Chief Financial Officer.

In FY2009, Berkeley Lab was authorized by DOE to establish a funding ceiling for the LDRD program of \$22.4M including General \& Administrative (G\&A) overhead, which equated to $3.5 \%$ of Berkeley Lab's FY2009 projected operating and capital equipment budgets. This funding level was provided to develop new scientific ideas and opportunities and allow the Berkeley Lab Director an opportunity to initiate new directions. Budget constraints limited available resources, however, so a little more than $\$ 19.6 \mathrm{M}$ was expended for operating expenses (3.23\% of actual Berkeley Lab FY2009 operating and equipment costs excluding ARRA funding).

In FY2009, scientists submitted 169 proposals, requesting about $\$ 31.2 \mathrm{M}$ in funding prior to assessing laboratory overhead. Ninety two projects were funded, with awards ranging from $\$ 19 \mathrm{~K}$ to $\$ 1,216 \mathrm{~K}$. These projects are identified in the Table of Contents. 


\title{
Accelerator and Fusion Research Division
}

\section{LB09007}

\section{Experimental accelerator $R \& D$ toward a future light source at LBNL}

\author{
Investigators: J. Corlett, F. Sannibale (P.I.), J. Staples, R. Wells, A. Zholents.
}

\section{Project Summary.}

The project has been to build hardware systems and associated test stands that critically impact the performance and cost of a future FEL (Free Electron Laser) light source at LBNL. In particular, we have developed a solution and produced a conceptual design for a novel electron photo-injector capable of generating the performance required by the operation of a high repetition FEL. Such studies are generally applicable to all future FELs designs, including but not limited to the one that LBNL is proposing. Existing electron sources fail to satisfy such requirements, and the development of such technology is a critical step towards the development of a high repetition rate FEL facility. The funds have been applied to fabrication of prototypes and procurement of systems for a cavity designed to operate in $\mathrm{CW}$ mode, with a goal of 20 $\mathrm{MV} / \mathrm{m}$ accelerating field at the cathode, and with $10^{-11}$ Torr vacuum pressure as required by high quantum efficiency cathodes.

Existing electron source technologies cannot meet the requirements of a high-repetition rate FEL light source. For example, DC guns do not reach the required beam energy; super-conducting guns are still in a "deep" R\&D phase trying to address several un-resolved issues; high frequency (greater than $\sim \mathrm{GHz}$ ) normal-conducting $\mathrm{RF}$ guns are limited in repetition rate by the maximum power that can be dissipated in the RF structure. At LBNL, we have developed a novel normalconducting RF gun scheme, where by significantly lowering the RF frequency (to $\sim 200 \mathrm{MHz}$ ) it is possible to reduce the power density on the cavity walls and thus operate the gun at very high repetition rates (potentially up to the RF frequency, several hundred $\mathrm{MHz}$ ). Additionally, the long RF wavelength allows for relatively large apertures in the cavity walls without distorting the fields, and thus to obtain the large vacuum conductivity necessary for achieving the targeted vacuum pressure.

\section{Accomplishments FY09.}

During this first year of LDRD, the following tasks have been achieved:

- The final design of the VHF cavity, core of the injector, has been completed. Two design reviews were held, with a committee of experts in the field (mostly external to LBNL), and the committee approved the design. The cavity is presently under construction in the workshops at LBNL. The fabrication is $\sim 60 \%$ completed, with most parts fabricated and brazing and welding stages expected to be complete in February 2010.

- A bid process to procure the $120 \mathrm{~kW}$ RF source has been completed and the contract was awarded. The source is about $40 \%$ constructed and will be delivered to LBNL at the end of February 2010.

- The preparation of the test area has been initiated and is in an advanced phase. The cavity will be tested at the Beam Test Facility (BTF), a shielded enclosure at the ALS. Preparations will be completed on time before the installation of the VHF cavity and the RF source (expected in spring 2010)

- Beam dynamics studies to simulate the performance of the VHF gun in a FEL injector have been initiated

- Design of the RF couplers to supply RF power to the cavity has been initiated

- Design of the RF distribution system has been initiated. 
Quantum information science with integrated color centers in diamond Principal Investigator: Thomas Schenkel ${ }^{1}$, AFRD, LBNL

\section{Project description:}

Color centers in diamond, like the nitrogen-vacancy center, exhibit amazing coherence properties, with spin lifetimes approaching milliseconds at room temperature. Color centers promise to revolutionize quantum information technology and our fundamental understanding of quantum coherence when they can be integrated into devices. To date, studies have been limited to uncontrolled, randomly distributed centers, and the coherence properties of many promising centers have not been characterized for lack of efficient center formation. We are forming arrays of color centers with unprecedented spatial resolution (few $\mathrm{nm}$ ) by ion implantation with scanning probe alignment and integrate center arrays into opto-electronic devices. We are also developing processes for efficient center formation and characterize coherence properties of new center systems.

Our unique setup for ion implantation with scanning probe alignment allows nondestructive imaging, alignment and ion placement with nanometer resolution and is ideally suited for the formation of color center arrays in diamond samples (which are usually small and rare). Single ion placement is enabled by the sensing of induced charge from single ion impacts, and we have formed first devices with tungsten electrodes on diamond for demonstration of single ion detection in diamonds. Samples are selected from commercial supplies of synthetic diamonds with ultra-low nitrogen content. We make use of the Imaging Facility at the Molecular Foundry for array characterization and collaborate very closely with Prof. Awschalom (UCSB) on the mapping and control of coherence properties using confocal laser spectro-microscopy, and optically detected magnetic resonance.

Following first center array formation with $80 \mathrm{~nm}$ dot sizes formation in the $1 \mathrm{st}$ project year, we succeeded in forming high quality NV-centers (with room temperature coherence times of $\sim 0.3 \mathrm{~ms}$ ) aligned to co-planar wave guides for nano-second scale driving of transmissions in single NV-centers (Fig 1). Studies of range profiles of implanted nitrogen ions revealed pronounced channeling effects, and we have developed strategies to mitigate these, which is important in order to achieve high levels positioning control over single centers.

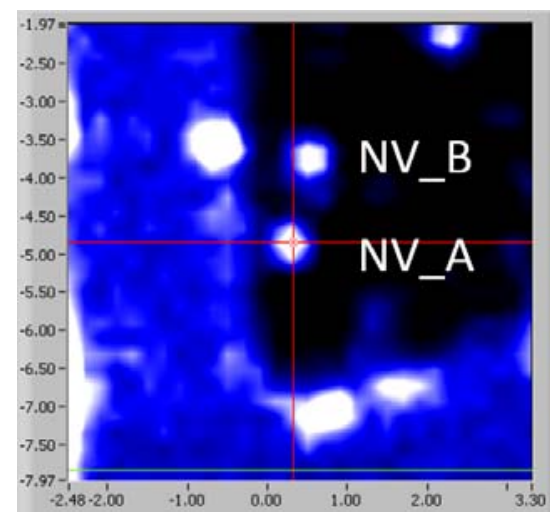

1 email: T_Schenke1@LBL.gov, 510-486-6674
Figure 1: Photoluminescence image of two 15-NV- centers placed close to a co-planar waveguide for efficient driving of spin transitions with microwaves. Image, courtesy of D. Toly, Awschalom group, UCSB. The imaging resolution in the confocal, photoluminescence microscope is about $400 \mathrm{~nm}$. 


\section{LDRD - LPADFEL - FY2009 report \\ Experimental demonstration of a laser-plasma-accelerator driven free-electron laser Principle Investigator(s): Csaba Toth, Carl Schroeder, William Fawley, Kem Robinson}

\section{Project Description}

The goal of this project is the demonstration and development of a free-electron laser (FEL) driven by the laser wakefield accelerator (LWFA) currently operating in AFRD's laser-plasma accelerator facility, the LOASIS Laboratory. The LDRD project consists of two stages: (1) The LWFA electron beam will be coupled to a $2.18 \mathrm{~cm}$-period undulator and the synchrotron radiation from three $(3 \times 22=66$-period $)$ undulator sections will be used to diagnose and characterize the LWFA electron beam. The intrinsic synchronization between the laser, electron beam, and radiation will be demonstrated. (2) Gain will be demonstrated via self-amplified spontaneous emission (SASE) in the XUV wavelength range. Fluxes on the order of $10^{13}$ photons/pulse are anticipated at saturation due to the high current $(>10 \mathrm{kA})$ of the LWFA beams. The LWFA-FEL will constitute a new class of compact laser-driven FEL [in which the conventional accelerator (10-100 m length) is replaced by a GeV-class laser-plasma accelerator (several $\mathrm{cm})$ ] producing ultra-short $(<50 \mathrm{fs})$ XUV pulses with high peak flux.

\section{Accomplishments}

We have achieved significant results in five important areas required for experimental demonstration of a LWFA-driven FEL:

(1) Further improved the electron beam quality of the LWFA via installing and fine-tuning an optical prepulse contrast filter in the laser beam (which generates the electron beam) and enhanced the pointing stability via a mirror alignment feedback system. We have started experiments with targets combining gas-jet+capillary using an integrated design and a hexapodbased capillary support system.

(2) Wavefront and reflectivity measurements performed at the waterjet test stand show sufficient flatness for laser reflection and revealed optimal laser intensity range for high reflectivity. The waterjet will provide a compact method to separate the laser and electron beam.

(3) The THUNDER undulator from Boeing is fully characterized by Hall-probe magnetic measurements at LBNL. The undulator and the alignment structures are installed in the fully renovated Bldg. 71 Cave-A (May 2009). The radiation interlock protection system has been installed, and Cave-A is ready for e-beam operation (Sep 2009).

(4) The special optical grating for the XUV spectrometer has been procured and installed, and the appropriate diagnostic CCD camera has been purchased. The housing of the spectrometer was manufactured, the components assembled, and vacuum and high voltage tests performed. Calibration tests are planned at ALS during the winter of 2009/2010.

(5) The expected spontaneous radiation emission was calculated using the code SPECTRA. Sensitivity to electron beam energy spread, emittance, and beam focusing (beam spot and divergence evolution through the undulator) were investigated. Matching the electron beam to the THUNDER undulator (60 micron beam radius in the undulator) using compact, high-field quadrupole magnets $(540 \mathrm{~T} / \mathrm{m})$ was examined using the particle tracking code GPT, assuming laser-plasma accelerator beam parameters at the exit of the plasma. Output FEL radiation was modeled using the FEL code GINGER. Higher energy electron beams were considered to generate soft $\mathrm{X}$-ray $(1 \mathrm{~nm})$ radiation. 


\section{Lorentz Compaction of Scales for Ultra-Efficient Simulation of Advanced Accelerators (and Other Systems). Principal Investigators: Jean-Luc Vay, William Fawley}

\section{Project Description}

The purpose of this project is to speed-up by orders of magnitude a class of first-principle computer simulations of complex physical problems, by recasting them into a Lorentz boosted frame of reference.

The design and understanding of modern experimental devices relies increasingly upon detailed computer simulation, but despite the awesome progress in the last decades in computer power and algorithmic developments, some problems remain extraordinarily difficult to model accurately. One such problem is laser wakefield acceleration (LWFA). Modeling 10-GeV stages for the LOASIS BELLA proposal in one-dimension using the Particle-In-Cell methods in the laboratory frame demanded as much as 5,000 processor-hours. A calculation of the same problem in a Lorentz boosted frame used only 3 processor-hours, a more than 1,500x speed-up. The new method would allow for 3-D simulation of this problem, which is ultimately needed but is currently beyond the state of the art using the conventional method.

During the course of this project, we will: (1) demonstrate, with actual 2-D or 3-D computer simulations of problems relevant to LWFA, electron clouds (EC) and free electron lasers (FEL) physics problems, orders of magnitude speed-up from a calculation in the laboratory frame to a calculation in the optimum Lorentz frame of reference; (2) for each targeted application, determine the range of validity for the Lorentz-boosted frame simulation results by comparison to laboratory frame simulations and/or well-known analytic results; (3) explore ways to perform calculations at optimum efficiency, regardless of the choice of the frame of reference; (4) explore other scientific applications which might benefit.

\section{Accomplishments}

In year 2, the method was successfully applied to the modeling of laser wakefield accelerators. Agreement has been demonstrated at the percentage level between simulations in a boosted frame and simulations in frames at $\gamma=1,2,5$ and 10 in $1 \mathrm{D}, 2 \mathrm{D}$ and 3D for a scaled $10 \mathrm{GeV}$ class stage. Simulations of full scale stages has been successfully performed with estimated speedup over 10,000x for frames boosted at $\gamma \sim 100$, over a similar calculation in the laboratory frame. A numerical instability developing in the boosted frame calculations in $2 \mathrm{D}$ and 3D was controlled by using a low dispersion electromagnetic field solver and broadband filtering. A $2 \mathrm{D}$ simulation of a $100 \mathrm{GeV}$ class stage was performed with an estimated speedup over 100,000x: the calculation which would take over 50 years in the laboratory frame took only a few hours in a boosted frame at $\gamma \sim 400$. In year 3, we intend to apply the model to design of low emittance LWFA stages, and develop ability to model trapping.

Our studies of simulations of FEL have concluded that if there are important physical phenomena that cannot be resolved properly by an eikonal code, a full electromagnetic simulation will be orders of magnitude faster in a boosted frame than in the laboratory frame. We have begun preliminary work of simulating CSR emission with the boosted frame method with Warp, examining the behavior of a high current, short electron beam traversing a simple dipole magnet. Our early results show that upon exit from the undulator, the electron beam shows the characteristic energy loss variation with longitudinal position that one expects from previous theoretical analyses of CSR. Further studies are underway. 
Emittance Manipulation and Beam Conditioning for FELs

Investigators: Marco Venturini, John Corlett, Gregory Penn, Ji Qiang,

Fernando Sannibale, and Alexander Zholents

\section{Project Description}

The operation of an x-ray FEL relies on electron beams with high peak current, low emittance and low energy spread, and uniform and controlled distribution of these quantities along the electron bunch. Production of these beams is a difficult and elaborate process. The purpose of the project is to investigate advanced concepts of electron beam generation, acceleration, compression, and transport for a next-generation free electron laser (FEL)-based light source that could be implemented at LBNL and meeting the goal of delivering high-energy $(2.4 \mathrm{GeV})$, high-quality beams $(\sim 1 \mathrm{kA}$ peak current with normalized emittance smaller than $1 \mu \mathrm{m}$ and relative energy spread at the $10^{-5}$ level) at a high rate $(1 \mathrm{MHz})$ for production of soft x-rays.

\section{Accomplishments}

The most significant accomplishment has been to finalize a conceptual design and layout for a linear accelerator capable of supporting a high brightness, high repetition rate, FELbased $4^{\text {th }}$ generation light source and document the work in a comprehensive report [LBNL Report LBNL 2670-E]. The proposed design, based on results from investigations carried out over the last and previous two years of this LDRD, offers a coherent and realistic picture of a high-performance machine that can be built with current or realizable technology and would meet the performance goals sought out by our studies. Activities of direct relevance for the proposed design conducted over the last year have included: an analysis of beam-jitter tolerances; the development of a concept for an RF-structure based device for collimation and/or control of the longitudinal bunch profile; investigation of space-charge effects in the low energy end of the linac past the injector; assessment of transverse multi-bunch instabilities. Of particular significance were the jitter studies, which included a model of feedback system, demonstrating that energy and arrival time fluctuations as low as $45 \mathrm{keV}$ and 15 fs respectively should be within reach.

Further activities have included investigations of RF compression, with attention paid to its impact on the microbunching instability, development of numerical tools for optimization of the design of the injector and preliminary studies for the injector lattice design, and participation in the LCLS commissioning at SLAC. 


\title{
High Brightness Photocathodes as Electron Sources for FELs
}

\author{
PIs: W. Wan, E. Pedersoli, M. Greaves, and H. A. Padmore \\ Project Description
}

Free Electron Lasers (FELs) offer instantaneous flux and brightness 10 orders of magnitude higher than a $3^{\text {rd }}$ generation synchrotron source like the ALS. They offer in addition pulse lengths of fsecs or less in comparison to the $100 \mathrm{psec}$ of a conventional synchrotron. However, in order to operate, the electron beam has to occupy a very small phase space volume, and in order to do this, presently operating and planned FELs start with a relatively hot beam from a laser driven photogun, and compress its phase volume by acceleration to high energy. In addition, present day cathodes have limited quantum efficiency, and so, limited by available laser power, FELs typically have low repetition rates. We are addressing these issues by investigation of a new generation of photocathodes, that have engineered electronic properties. The aim is to develop a cathode that produces a beam with very low transverse energy spread; this will enable higher photon energies from a FEL, or simply reduce its scale. In addition, we aim to increase the quantum efficiency to enable $\mathrm{MHz}$ repetition rate. Successful development of photocathodes would result in reduced cost and technical risk, and enhanced opportunities, for a new FEL facility to be built at LBNL.

\section{Accomplishments}

This year the work focused on the evaluation of the benefits of off-axis illumination of the cathode using $\mathrm{p}$ - polarized light. The cathodes investigated were Copper, due to its use in the world's $1^{\text {st }}$ hard x-ray free electron laser, the LCLS. The $1^{\text {st }}$ set of experiments measured the electron yield of $\mathrm{Cu}$ (111) single crystals as a function of photon energy and polarization, using a photon energy close to the work function. We discovered that at the low photon energies used in photoinjector, the photoelectron yield increased by up to a factor of 15 when using the optimum incidence angle of 70 degrees, compared to normal incidence. The effect of this large increase in quantum efficiency is a commensurate reduction in the required laser power for a determined pulse charge in the FEL photoinjector. As this power is a limiting factor in high repetition rate operation, this gain in QE could be highly beneficial. We also determined the sensitivity of the effect to atomic order of the surface, through the artificial creation of disorder $n$ bombardment. It was found that the vacuum metal interface has to be sharp, but is not strongly dependent on surface oxidation. This work opens the way to other high QE metallic photocathodes with perhaps even higher enhancement. The effect appears to be due to the breakage of symmetry at the surface causing sufficient electron momentum uncertainty to couple all initial and final states separated by the photon energy, ie. momentum conservation is removed, thus significantly increasing the possible number of optical transitions and electron yield. We also investigated the effect of microstructure in real LCLS cathodes, using UV induced photoemission electron microscopy. This revealed a complex structure with large domains of bright and weak emission; this was found to correlate with micro-x-ray diffraction studies of the domain structure of the material which revealed a 2 phase material of 111 and 100 grains. This spotty emission has implications for emittance in dense beams and should be avoided by use of single crystals. 
FEL Concepts for Multiple Independent X-ray Beamlines

Investigators: William Fawley, Gregory Penn, and Alexander Zholents

\section{Project Description}

We propose to address fundamental issues and develop concepts for a next generation, FEL-based light source at LBNL. To meet the diverse interests of experimentalists, we consider an array of free electron lasers (FELs) where each FEL is tailored to individual experimental needs for average and peak brightness, pulse repetition rate, resolving power, and wavelength. Thus the entire ensemble of FELs will provide a high degree of flexibility for choosing photon energy, $\mathrm{x}$-ray pulse duration, $\mathrm{x}-$ ray signal bandwidth and polarization. A variety of FEL configurations such as SASE, HGHG, self-seeded and seeded by HHG signal will be studied. The electron beam switch yard distributing electron bunches into various FELs will be designed.

Accomplishments

This year studies were inspired by a proposal to use stimulated x-ray Raman spectroscopy to probe electron dynamics in molecules using x-ray pump and x-ray probe ultra-short x-ray pulses at different wavelengths. In order to do it, we proposed a method of creating a sequence of two attosecond x-ray pulses in a free electron laser by optical manipulation of electrons located in two different sections of the electron bunch. Basically, we combine two recent ideas, i.e. methods of the current enhanced self amplified spontaneous emission (ESASE) and echo enabled microbunching (EEHG). Due to the ESASE we obtain a short gain length for the FEL process and due to EEHG we obtain microbunching of electrons at the x-ray wavelength to seed the FEL process. Remarkably, by slight adjustment of the intensity of two laser pulses interacting with electrons we could obtain microbunching at two different wavelengths, i.e. two colors. What is also remarkable is that the two x-ray pulses are precisely synchronized and that the time interval between the two pulses is tunable in the range from a few femtoseconds to a hundred femtoseconds with better than 100 attoseconds precision. The energy of each x-ray pulse can be of the order of $100 \mathrm{~nJ}$ and the pulse width of the order of 250 attoseconds. The carrier frequency of each pulse can be independently tuned to a resonant core electron transition of a specific atom of the molecule, in order to view the movement of excited electron states within the molecule.

In a separate activity, we performed the feasibility studies for a soft x-ray free electron laser oscillator. The problem there is a very limited choice of mirrors with a good reflectivity. This severely limits the FEL tunability making it practically useless. To resolve the problem we came up with an idea of using two fixed wavelengths oscillators operating with good mirrors, and achieving needed tunability by optically manipulating the electrons to produce microbunching at a much shorter wavelength then in either of two oscillators. Basically, we proposed an extension of the EEHG technique into the regime of the FEL oscillators. To date, we developed 1D analytical description of the entire system and used it to create a fast MATLAB code to perform simulation studies. We obtained preliminary results that confirm our original expectations. 


\title{
Advanced Light Source Division
}

\author{
Optimization of Flux Pinning in Type II Superconductor Based Magnets for Soft X-ray \\ Scattering Applications \\ Principal Investigator(s): Elke Arenholz, Soren O. Prestemon, YiDe Chuang
}

\section{Project Description}

We proposed to develop high field magnets $(\mathrm{H} \geq 5 \mathrm{~T})$ for soft $\mathrm{x}$ ray magnetic scattering applications based on flux pinning in type II superconductors. Type II superconductors can trap magnetic flux and retain it even at zero applied field if cooled in an external field through their transition temperature or when subjected to a field cycle. They therefore can be used to replace permanent magnets in applications - like soft $\mathrm{x}$ ray magnetic scattering experiments on novel magnetic materials - where much higher fields are needed and low temperatures are possible. Such flux pinning superconductors have been studied for years and even been commercialized for some applications. However, they have never been used in scattering experiments.

\section{Accomplishments}

It was the goal of the original FY2008 LDRD and its FY2009 continuations to demonstrate that flux pinning in type II superconductors is a viable and powerful method to provide magnetic background fields of $5 \mathrm{~T}$ or more for soft $\mathrm{x}$-ray scattering experiments.

With funding from the FY2008/2009 LDRDs a test and characterization set up for the detailed determination of flux pinning in the YBCO discs was designed and built. A photograph of the set up is shown in Figure 1. A commercial superconducting magnet provides axial fields up to $9 \mathrm{~T}$ in a 2" room temperature bore. A custom vacuum chamber is installed inside the magnet bore providing an UHV environment for the flux pinning experiments. The YBCO discs are mounted on a custom cryostat installed in the center of the magnet. A Hall probe inserted from the opposite side in the magnet bore is scanned near the YBCO surface to determine the spatial distribution of the pinned flux.

We identified Y-Ba-Cu-O (YBCO) discs (20 mm diameter, $8 \mathrm{~mm}$ thick) provided by $\mathrm{R}$. Weinstein, University of Houston, as most suitable materials for our soft x-ray scattering applications since up to $80 \%$ of the field applied during field cooling is reliably pinned in the YBCO discs by defects after removal of the external field at $5 \mathrm{~K}$. For higher temperatures the fraction of the external field trapped in the YBCO disc is less but still exceed $50 \%$ for temperatures up to $30 \mathrm{~K}$. Moreover, flux creep, i.e. the loss of pinned flux due to thermal fluctuations, can be completely eliminated by lowering the temperature of the YBCO disc in zero field after the field cooling process leading to very stable experimental conditions. The field distribution near the YBCO surface after the temperature field cycle as well as its dependence on

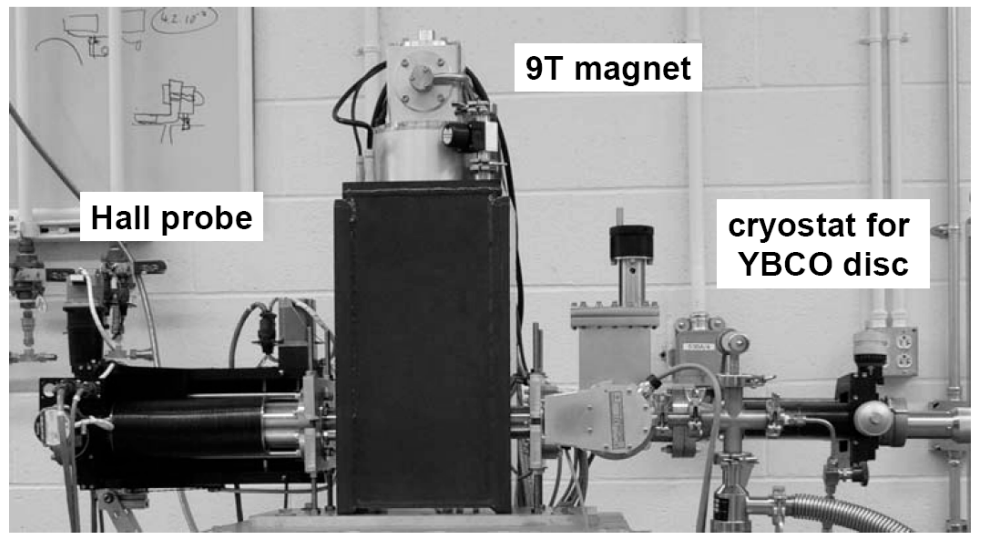
cooling field and temperature has been studied in detail.

Figure 1: Test set up for the characterization of flux pinning in YBCO. YBCO discs are mounted on custom cryostat installed in the center of the 9T magnet. A Hall probe inserted from the opposite side in the magnet bore is scanned near the YBCO surface to determine the spatial distribution of the pinned flux. 


\section{Building In-situ Electronic Structure Study Capability with Photon-in/Photon-out Soft-X-Ray Spectroscopy \\ Principle Investigator(s): Jinghua Guo}

\section{Project Description:}

The purposed project is to build in-situ electronic structure study capability of the chemical reactions in photon-in/photon-out soft-x-ray spectroscopy on beamline 7.0 at ALS. The goal is to demonstrate a unique way to handle the real systems (gas-, liquid-phase samples, wet surfaces and interfaces) for electronic structure studies. The focus in science will be on understanding the oxidation and reduction mechanism of metallic nanoparticles in catalytic reactions, and in-situ electronic structure studies of electrochemical reactions in general, which will provide in depth understanding of nanoscience and renewable energy science.

The photon-in/photon-out soft x-ray spectroscopy can be used to study different chemical species of interested in depth, or under the presence of electric and/or magnetic fields, or under wet condition and ambient pressure. In this project, the in-situ electronic structure study using photon-in/photon-out soft-x-ray spectroscopy has been realized by constructing a gas reaction cell for the electronic structure study.

The proposed effort requires the new experimental equipment to be built. The gaseous and liquid phase samples will be maintained in cells inside the vacuum chamber with windows to allow the penetration of soft x-rays. We have built the chemical cells for in-situ photonin/photon-out soft-X-ray spectroscopy studies. The cells include the gas reaction cell for in-situ electronic structure studies and the liquid cell for electrochemical process and make it compatible with vacuum environment, and perform a test experiment of in-situ electronic structure studies of nanoparticles under catalytic reactions and electrochemical redox reaction in solutions.

\section{Accomplishments:}

We have completed an engineering design and fabrication of the gas reaction cell with an operating temperature range of $80-550 \mathrm{~K}$. In commissioning of the gas reaction cell, we were able to reduce a thin layer of $\mathrm{CoO}$ on a piece of $\mathrm{Co}$ metal, and also reduce the oxidized $\mathrm{Co}$ nanoparticles under a flow of 20 torr $\mathrm{H}_{2}$ at a temperature of $300{ }^{\circ} \mathrm{C}$. The metallic and oxidized Co bulk and nanoparticles were characterized with soft x-ray spectroscopy.

A new liquid flow cell for electronic structure study of liquids was already fabricated. An electrochemical reaction was tested with a Co metallic film on the silicon nitride membrane window as electrode, and $\mathrm{Ag}$ as reference electrode and $\mathrm{Pt}$ as counter electrode. Oxidized Co was detected by soft x-ray absorption spectroscopy. Also, a 100-nm Cu film on silicon nitride membrane window was also tested. A number of redox processes was realized while oxidation and reduction of $\mathrm{Cu}$ were characterized by soft $\mathrm{x}$-ray absorption spectroscopy. 


\section{Soft X-ray Scattering As a New Probe of Polymer Systems}

Alexander Hexemer, Howard Padmore, Zahid Hussain, Jeff Kortright, Nitash Balsara, Rachel Segalman, Ting Xu, Ed Kramer, Sunil Sinha, Harald Ade, Rex Hjelm, Lynn Loo, Thomas Russell

\section{Project Description:}

RSoXS is a new X-ray probe that can be used to analyze polymers and related hard and soft condensed matter. It combines the conventional hard x-ray scattering with the unique chemical sensitivity by using soft $\mathrm{x}$-ray near the absorption edge, which can provide enhanced and tunable contrast between different components. It simultaneously provides chemical information, spatial information, and information about interfaces. By using this new technique, researchers will be able to understand how different structures can generate different functional properties in these materials. For example, polymer scientists will be able to understand how changes in the heterogeneous morphology of a block copolymer can yield more light in an organic light emitting diode, or more efficiency in a photovoltaic. The ability to understand these structurefunction relationships is crucial if researchers are to develop a new generation of materials and devices, and progress is currently limited by a lack of high-resolution analytical probes. RSoXS should fill the need for a high-resolution spatial probe based on chemical composition.

Accomplishment:

During FY09, we have been constructing a dedicated and optimized instrument for resonant scattering experiment. An existing reflectometer chamber at BL 6.3.1 has been customized, modified, and upgraded to accommodate a large variety of organic thin film samples and scattering geometries including transmission, specular and off-specular reflection, and grazing incidence geometries that allows users to study both laterally- and depth-resolved structure in ultrathin polymer films with essentially any form of chemical heterogeneity and degree of order with nanometer resolution. With the current setup, we collected resonant soft x-ray scattering data in different geometries on various systems including block copolymer nanostructures, organic light emitting diode, and polymer photovoltaics, etc.

Currently, we are in the process of transferring this scattering chamber to a new location at BL11.0.1. BL11.0.1 is a state of art elliptically polarized undulator beamline that covers the energy range of 200-1300 eV. The scattering chamber is installed as a branchline shared the optics with another endstation that is separated using a bending mirror. Compared to our current location at BL6.3.1, we will have much higher flux, much better energy resolution, which will significantly improve our ability to perform high precision soft x-ray scattering measurements. 


\title{
High quantum yield multi-alkali cathodes for psec pulsed electron sources
}

\author{
PI: H. Padmore
}

\section{Project Description}

Many experiments require sources of psec dense electron beams, from those to be used for electron diffraction to free electron lasers. The generation of these beams is typically done using laser excitation on a metallic substrate. While robust, these cathodes are inefficient, typically having a quantum yield in the UV of 1e-4. The goal of this work therefore is to design and test new types of cathode based on high yield semiconductors. The system we have chosen to work with was discovered 40 years ago, but has only had limited success in pulsed electron sources due to issues related to dark current and fragility of the emitting surface. The main system we plan to work with is $\mathrm{K} 2 \mathrm{CsSb}$, which in the green part of the spectrum can have a $\mathrm{QE}$ of $10 \%$. Our goal is to understand the growth and surface chemistry of this system so that it can be reliably used in high current pulsed electron applications.

\section{Accomplishments}

In initial work at the end of FY09, we have purchased the necessary evaporator to allow Molecular Beam Epitaxy deposition of $\mathrm{Cs}, \mathrm{K}$ and $\mathrm{Sb}$. This was installed and is in the process of being commissioned in a UHV chamber. We also designed a sample heater to allow $1100 \mathrm{~K}$ temperatures to be reached, at the same time providing electrical isolation and rapid cooling. As the final step in production is deposition and reaction of Cs, after manufacture at $430 \mathrm{~K}$, we need to cool very rapidly back to room temperature. Our new heater allows us to do this by blowing air at high pressure through thin stainless steel cooling lines and into the hollow heater block. The experimental chamber has been set up with a film thickness monitor and various laser based tools for monitoring of thickness through changes in reflectivity. We have also just installed a tunable visible light monochromator to enable measurements of electron yield. 
An improved electron detection system for

"Extreme" angle-resolved photoemission experiments

Principal Investigators: Eli Rotenberg and Aaron Bostwick

\section{Project Description}

The purpose is to implement a new scheme for measuring the angular distribution of electrons for angle-resolved photoemission spectroscopy (ARPES) in "extreme" conditions such as ultralow temperature and nanoscale probe size. Such a lens is essentially an angular electron microscope because it collects angular, not spatial images. Its development not only supports the user program at the nanoARPES endstation at the ALS (part of wave 1 of the ALS strategic plan) but can also be commercially viable and could be licensed should a patent be filed for it. We have developed a working unit with $12 \times 12^{\circ}$ scanning range and found no significant aberrations. Our goal is now to develop a new lens with $30 \times 30^{\circ}$ acceptance in order to sample a full unit cell in electron-momentum space.

We will develop the software for optimization of electron paths using ray-traced electron paths generated by standard software packages. We will demonstrate through simulations the extension of the previous prototype lens' operating range from $12 \times 12$ to $30 \times 30^{\circ}$. The designs are intended as the driver for future fabrication of a working model that can be installed at an endstation at the ALS. We will explore designs for an even larger angular range that can be built in later years.

\section{Accomplishments}

Our main accomplishment was to successfully simulate an angular scanning lens capable of nearly the desired design range. The figure below shows the results. The left panels are electron trajectories projected onto the central plane of the lens column, and the right panels show the final positions on the exit slit plane. The bottom, middle, and upper panels show the operation of the angular microscope in low, intermediate, and high deflection. These data show that it is possible to transfer different planes of the source electron distribution through a narrow
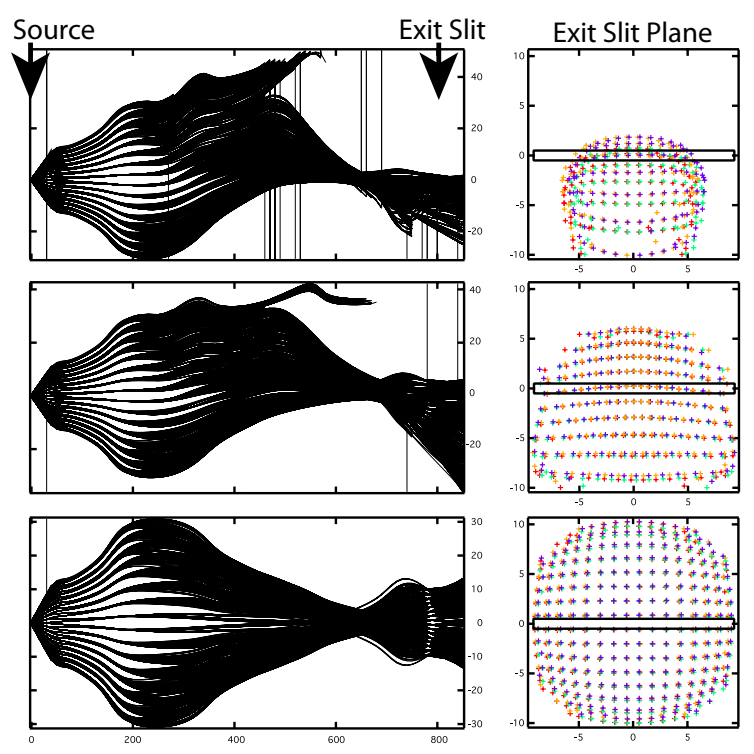
slit in the center of the detector plane as desired. This demonstrates the design goal for total angular range can be met. Since a large source size (1mm) was used in the simulations, the results demonstrate satisfactory operation not only for nanoARPES but also conventional ARPES setups.

Our present work focuses on reducing the remaining aberrations, and on implementing a prototype microscope which can be installed at the ALS for testing and spectroscopy experiments.

Figure. (left panels) Simulated electron trajectories for a 1 $\mathrm{mm}$ source projected onto the centerral plane of the lens with $\pm 15^{\circ}$ angular distribution. (right) Projected landing sites on the exit slit plane. Bottom to top rows correspond to three different electron deflections. 
Development of a laser goniometer for x-ray and electron diffraction microscopy Principal Investigators: David Shapiro and Yiqiong Zhao

\section{Project Description}

Coherent x-ray diffraction microscopy is a lensless microscopy that can produce high resolution images of materials from only a measurement of their far-field $\mathrm{x}$-ray diffraction pattern. In principle, the lack of lenses means that the achievable resolution is limited only by the $\mathrm{x}$-ray wavelength and the scattering angle to which adequate photons are counted. The $\mathrm{x}-$ ray diffraction phase problem is solved through adequate sampling of the diffraction pattern followed by an iterative phase retrieval computation. Reliable sampling of the pattern requires that the coherence length of the illuminating radiation be at least twice as big as the sample of interest and that the sample be surrounded by a zero scattering region. In practice, this is very difficult to achieve and more often than not, parasitic scatter from the specimen's support membrane contributes significantly to the recorded diffraction pattern. This parasitic scatter can have different effects depending on its nature but in general it will make the phase problem more difficult to solve resulting in lower resolution images and a less reliable imaging method.

The goal of this project is to develop a laser trap ("laser tweezers") that can levitate, translate, and rotationally orient micron sized particles in a gaseous environment or vacuum so that they may be used in x-ray diffraction microscopy experiments without a support membrane. This will result in a near total reduction of parasitic scatter from the soft x-ray diffraction patterns and result in a more reliable and higher resolution imaging system. It will provide the possibility of few-nm resolution imaging, in three dimensions, of several-micron sized material sciences samples. In the biological sciences, it offers the opportunity to combine established optical techniques, such as fluorescence microscopy and Raman spectroscopy, with high resolution xray imaging. We will use a standard optical tweezers design that is modified to allow delivery of aerosolized particles to the trap and we will develop a means of dynamically adjusting the illumination to allow controlled rotations of the trapped particles.

\section{Accomplishments}

We have developed the laser trapping system which is capable of holding, translating, and rotating, 3 micron-sized particles in air.

The rotational dynamics of laser-trapped three-micron gold particles were studied in detail and the measurements are accepted for publication in Optics Express. We demonstrate the presence of a helical phase in a focused circularly polarized laser beam which allows for the transfer of orbital angular momentum to non-birefringent trapped particles. This conversion of spin to orbital angular momentum through focusing is predicted by theory and was accurately measured with our laser trap. The transfer of orbital angular momentum can be controlled via the polarization state of the laser and therefore allows for rotational control of the trapped sample.

An analogous laser trapping system was installed at beamline 9.0.1 of the ALS and was aligned to the $\mathrm{x}$-ray beam for diffraction measurements. This system provides a high numerical aperture in-vacuum visible and infrared light microscope. Initial x-ray experiments were not successful due to failure of the detector and the sample delivery scheme in vacuum. Experiments will proceed with a general user proposal. 
Ultra-high resolution optics for soft x-ray inelastic scattering Principal Investigator: Valeriy V. Yashchuk

\section{Project Description}

This work started as an LDRD project in 2007 and completed its goal in FY2009 to demonstrate and establish the technology required for ultrahigh resolution Resonant Inelastic soft X-ray Scattering (RIXS). RIXS is one of the most important potential new probes in electronic structure determination due to its elemental selectivity, high momentum resolution, and ability to probe bulk materials. However, important advantages of the method are limited due to energy resolution limits imposed by traditional grating optics, especially at soft x-ray energies. For example, energy scales imposed by collective phenomena in correlated electronic systems dictate that meV energy resolution is required for experiments at 500-1000 eV energy, requiring a resolving power of about $10,{ }^{6}$ unachievable with conventional optics.

We have proposed and worked to realize a revolutionary new way to construct gratings with ultra-high groove density, potentially up to $50,000 \mathrm{~mm}^{-1}$ to get to the high resolving powers required. We fabricated such a grating by deposition of a soft x-ray multilayer on a substrate which is a blazed saw-tooth grating (echellette) with low groove density. Subsequent polishing applied to the coated grating removes part of the coating and forms an oblique-cut multi-line structure that is a sliced multilayer grating. The resulting grating has a short-scale periodicity of lines (bilayers), which is defined by the multilayer period and the oblique-cut angle. Additional increase of the effective groove density is due to the optimization of the grating parameters for higher order diffraction.

\section{Accomplishments}

Our most significant accomplishment has been to develop a reproducible technological fabrication cycle for high resolution diffraction gratings according to the suggested approaches and the required tolerances (numerically simulated and optimized in the course of FY2007 work on the project) on different steps of grating fabrication. In FY2008, we succeeded in fabricating and testing a Sc/Si sliced grating, which is a first prototype of a super-dense grating suitable for EUV applications. Diffraction experiments with the ALS BL6.3.2 spectrometer provided a lower limit (due to the resolution of the spectrometer rather than the grating) of $\sim 400$ for the resolution of the sliced grating at a diffraction efficiency of $\sim 7 \%$ for the optimized 38th order assigned to the echellette grating of $10 \mu \mathrm{m}$ period.

In FY2009, we have completed optimization of grating fabrication, in particular, anisotropic etching of a Si wafer, developing an echellette substrate with an atomically smooth surface and close to an ideal saw-tooth-like profile, and the multilayer deposition on echellette substrates. Based on the optimized fabrication processes, a number of highly efficient diffraction gratings optimized for EUV light $(\sim 100 \mathrm{eV})$ have been fabricated and tested. The gratings with a $200 \mathrm{~nm}$ period of the blazed grating substrate coated with 20$30 \mathrm{Mo} / \mathrm{Si}$ bilayers with a period of $\sim 7 \mathrm{~nm}$ have demonstrated excellent dispersion in the third order (effective groove density of 15,000 lines per $\mathrm{mm}$ ) with a diffraction efficiency of more than $35 \%$. That is, to the best of our knowledge, the highest effective density and efficiency observed to date worldwide. 
X-Ray Optical Metrology for Coherence-Preserving Adaptive Optics Principal Investigators: Kenneth A. Goldberg, Valeriy Y. Yashchuk

\section{Project Description}

The goal of this project is to develop ultra-high accuracy mirror metrology methods to enable $\mathrm{x}$-ray nano-focusing and coherence preservation for synchrotron and free-electron laser (FEL) beamlines. Nano-focusing, with spot sizes at or below $50 \mathrm{~nm}$, is an essential technological requirement for x-ray nano-science projects being planned worldwide. X-rays are focused using atomically smooth, glancing incidence mirrors, bent or polished into precise, curved shapes with 100-nano-radian tolerances. Metrology limitations have caused a performance plateau that makes it exceedingly difficult to achieve and maintain nano-focusing on x-ray beamlines. Furthermore, environmental factors such as temperature, vibration, and mechanical drift frustrate stable alignment. Relative to Fresnel zoneplate lenses, which are also used for x-ray focusing, bent or curved mirrors have the significant advantages of high efficiency, achromaticity, high power-load tolerance, temporal coherence preservation, and long working distance.

We are creating both the experimental and theoretical infrastructure to perform and analyze these tests at the required levels of sub-10-nm and sub-100-nrad accuracies. Our approach is to integrate ex-situ optical testing and in-situ x-ray wavefront sensing on high quality x-ray optics. Following pre-alignment with high-precision optical metrology techniques (e.g. long-trace profilometry), mirrors are transferred to the beamline where they are subjected to a series of at-wavelength ( $\mathrm{x}$-ray) tests and alignment iterations utilizing successively higher resolution until diffraction-limited performance is achieved. These tests include the scanning-slit and traditional Hartmann tests, knife-edge testing, shearing interferometry, and coherent diffraction reconstruction. These methods share compatible hardware, and yield quantitative wavefront aberration information with increasing levels of accuracy and sensitivity. This project leverages proven successful methods developed for extreme ultraviolet lithography research, also conducted at LBNL.

\section{Accomplishments}

We have created a dedicated metrology beamline end-station, including a universal test-chamber with various diagnostic instrumentation, to develop and hone the in-situ x-ray techniques. Separately, our recent optical experiments have validated the hypothesis that temperature strongly affects the surface profile of bent mirror substrates. To mitigate this effect we created and validated a new, temperature-stabilized design of bendable mirrors for use in these and future experiments. Nano-fabricated diffractive optical elements for the object plane and the focal plane have been designed and produced. With the chamber assembled, and all necessary parts in hand, we are performing final component testing and vacuum system qualification before starting the $\mathrm{x}$-ray testing.

We have also developed a new theoretical framework for the optimization of Kirkpatrick-Baez mirror alignment in one and two dimensions, and we are exploring novel mathematical methods of recovering the aberrated wavefront phase with larger aberration magnitudes than traditional phase-recovery techniques allow.

The techniques developed by this work can be exported to improve the performance of existing and future x-ray beamlines worldwide. 


\title{
Chemical Sciences Division
}

\section{Chemical reactions at liquid/vapor interfaces probed by photoemission spectroscopy}

\author{
Principal Investigators: Hendrik Bluhm and Kevin R. Wilson
}

\section{Project Description}

The goal of this project is to build a novel instrument for the investigation of chemical reactions at liquid/vapor interfaces under atmospherically and environmentally relevant conditions. The properties of liquid/vapor interfaces strongly influence heterogeneous processes in atmospheric and environmental chemistry. Up to now little is known about the concentration of solution phase species at the liquid/vapor interface, which can significantly differ from the bulk solution concentration and is an important quantity in the modeling of heterogeneous reactions at liquid/vapor interfaces. A direct measurement of the liquid/vapor interface under atmospherically and environmentally relevant conditions is difficult due to the lack of quantitative experimental techniques that are surface-sensitive, chemically specific and can operate at elevated pressures in the Torr range. By combining synchrotron-based ambient pressure photoemission spectroscopy (APPES) and a liquid droplet train into a single experimental setup we will address this need and provide quantitative information about the chemical nature of the liquid/vapor interface under varying conditions of bulk concentration and temperature, as well as gas phase composition and pressure.

We have planned our experiments in two stages. In the first phase we have implemented a droplet train generator in the ambient pressure photoemission endstation at beamline 11.0.2 at the Advanced Light Source. This setup allowed us to optimize the experimental parameters and, in addition, to perform first experiments on solution/vapor interfaces. We are now finishing the setup of a dedicated machine that will allow us to vary the interaction time between droplets and gas molecules. This will enable us to investigate the kinetics of heterogeneous reactions that have great relevance to atmospheric chemistry but are currently incompatible with conventional ultra-high vacuum systems (e.g., $\mathrm{HNO}_{3}, \mathrm{H}_{2} \mathrm{SO}_{4}$, and $\mathrm{HCl}$ ).

\section{Accomplishments}

In the first stage of this project we have successfully implemented a droplet train generator in the existing APPES endstation at beamline 11.0.2. The test measurements showed that for the successful performance of droplet train experiments an increase in the signal-to-noise level is essential, which can mostly be achieved by an improvement of the differentially pumped lens system of the PES instrument. To that end a new concept for the layout of the differentially pumped lens system was developed in collaboration with the supplier. This new lens system has a 5 times larger acceptance angle, which will lead to an increase in count rate by a factor of 10 . A further increase in signal is expected from the utilization of deflector plates in the first lens stage, which allows one to adjust the electron beam trajectories as a function of kinetic energy and will reduce losses at the upstream apertures. In addition, this lens system features a miniature gate valve inside the lens column, which allows to vent the sample cell without exposing the analyzer to ambient pressures, a feature that will increase the efficiency of the experiments greatly, which is in particular important for experiments at a synchrotron.

During the last year we have in addition performed test experiments on the detection of solid aerosols particles using an aerodynamic lens. This setup, in combination with the droplet train technique, will allow us to sample a wide range of aerosol surfaces, from liquids to solids as well as liquid coatings on solid surfaces. A paper that describes the results of the aerodynamic lens/APPES test measurements and a description of the technical details has been accepted for publication in Review of Scientific Instruments. 


\section{Probing Transient Molecular Entanglement using \\ Femtosecond High Resolution Delayed-Field Coincidence Imaging \\ Principle Investigator(s): Oliver Gessner}

\section{Project Description}

This project is aimed to study the transient entanglement of the constituents of a molecule during unimolecular dissociation in real-time. It will explore the very heart of chemical bonding: the boundaries and the transition between separate particle and unified molecular behavior. The quantum mechanical nature of chemical bonding over potentially macroscopic distances and the conditions to create entangled particles through unimolecular dissociation in the gas phase will be investigated.

A newly designed high-resolution delayed-field photoelectron-ion coincidence imaging spectrometer will be used in combination with the high repetition rate ultrafast soft X-ray source of the Ultrafast X-ray Science Laboratory (UXSL) at LBNL. The studies will constitute a significant contribution to the UXSL endeavor in becoming one of the world's leading centers for ultrafast X-ray science. They will aid in paving the way for future science performed at ultrafast X-ray light sources like the Stanford LCLS, and help to define benchmark requirements for a new FEL-based ultrafast X-ray source at LBNL.

\section{Accomplishments}

A key component of the experiment, the time- and position-sensitive ion detector has been successfully taken into operation. It has been implemented in a Wiley-McLaren type ion time-of-flight (TOF) spectrometer that is mounted opposite to an existing electron Velocity-Map Imaging (VMI) spectrometer. This configuration allows for complementary measurements of electron and ion emission angular distributions that can be performed almost simultaneously.

First experiments on helium nanodroplets have demonstrated the power of the novel detector technique to record mass-, angle-, and kinetic energy-resolved ion spectra in a non-dispersive fashion. This multiplexed ion detection technology significantly increases the achievable count rates in photoionization experiments which is a prerequisite for the planned coincidence experiments using soft x-ray light sources with moderate average photon flux.

A new postdoctoral associate has joined the project. Dr. Oliver Bünermann, who has recently received his Ph.D. from the University of Freiburg, Germany, holds a Research Stipend of the Deutsche Forschungsgemeinschaft (DFG, German Research Foundation). Within the first 7 months of his appointment he has designed and implemented the new ion spectrometer described above and has acquired all necessary skills to operate the UXSL Chemical Dynamics lab state-of-the-art high-power, high repetition rate femtosecond laser system and the associated high-order harmonics source. His results will be presented at several international meetings in 2010 .

An international patent application has been filed by the LBNL patent group for an ultracompact VUV spectrometer and beam-profiler that has been developed in the course of this LDRD project. The technology is currently being advertised for licensing by the LBNL technology transfer group. 
Development of in situ cells for Reactive Spectroscopic and Microscopic Studies Principal Investigator(s): Mary Gilles

\section{Project Description}

This proposal is to develop in situ cells for scanning transmission x-ray microscopy (STXM) studies of nanoscale systems relevant to energy production. Potential applications include: biomass energy production; syngas production; and the development/improvement of solar cells. In situ cells are critical components for advancing studies in a wide range of fields; atmospheric chemistry, solar cells; electrochemistry (i.e. batteries), soil chemistry, magnetic systems, polymer processing, and catalysis. Commonalities between all of these include the need to understand detailed changes in chemical composition on the scale of 10's of $\mathrm{nm}$ to microns in reactive environments.

The goal is development of several in situ cells that are robust and can be adapted to other experimental platforms at the Advanced Light Source, including Fourier transform infrared spectroscopy, X-ray fluorescence microprobe techniques, or bulk near edge x-ray absorption fine structure (NEXAFS) measurements. Since laboratory based ultrafast (femto or attosecond) transient absorption experiments share similar sample requirements with STXM, these cells could be readily adapted to laboratory based experiments.

Essentially three types of cells will be developed: a micro fluidic cell, a temperature controlled reactor (reactive gas flow and varying relative humidity); candidates for the third cell include either a micro fluidic fuel cell or an electrochemical cell.

\section{Accomplishments}

A post-doc with micro-fabrication and fuel cell expertise was hired in early 2009 and in the spring a graduate student performed experiments on a silicon oxide polymer system that would serve as a suitable prototype in situ test case.

Two in situ cells were designed and manufacture is nearly complete on both prototypes: 1) a microfluidic cell with a path length of 1.5 microns and 2) a STXM reactor cell incorporating heating and gas flow.

The STXM reactor cell is based on a ceramic holder with replaceable windows. One of the windows incorporates a spiral type Pt heater on the silicon nitride membrane. These are in the final fabrication stage. As the temperature gradient across the silicon nitride membrane is expected to be large, chemical methods to characterize this gradient with a few hundred nanometer spatial resolution were designed. The equipment and chemicals necessary for these measurements recently arrived.

The micro fluidic cell design incorporated ideas from other research groups and fabrication for the flow cell is nearly complete. Experiments examining the distortion of the path length with flow were designed and expected to begin shortly. After characterizing this larger path length cell we will move step wise to shorter path lengths. 
Dynamics of homogeneous catalysis reactions investigated with transient two- dimensional infrared spectroscopy

Principle Investigator: Charles B. Harris

\section{Project Description}

Organometallic compounds have broad applications in synthetic chemistry, particularly as homogeneous catalysts. A full understanding of the mechanisms and associated dynamics of organometallic reactions allows new synthetic routes to be designed and current syntheses to be improved. Direct experimental observation of these dynamics is often the best way to determine the details of these transformations. We have previously studied organometallic catalytic systems using transient one dimensional spectroscopy, in which a reactions is photoinitialized by a UV pulse and is then probed by an IR. We are currently extending our work to the study of photochemical reactions using transient 2D-IR (T2D-IR). Thorough analysis of the T2D-IR spectra can give information on the structure of not only the transient intermediates but also the transition states, details that conventional spectroscopic techniques cannot provide. In order to show the efficacy of this technique, we are performing proof of principle experiments in which we are studying the photolysis of iron pentacarbonyl to iron tetracarbonyl. We then intend to use our expanded multidimensional capabilities to study important homogeneous catalytic reactions such as selective $\mathrm{C}-\mathrm{H}$ bond activation by a novel boryl complex, $\mathrm{Cp} * \mathrm{~W}(\mathrm{CO})_{3}(\mathrm{Bpin})(\mathrm{pin}=1,2-$ $\left.\mathrm{O}_{2} \mathrm{C}_{2}-\left(\mathrm{CH}_{3}\right)_{4}\right)$ in neat pentane solution.

\section{Accomplishments}

In the past year we have made several upgrades to our apparatus necessary to perform T2D-IR experiments, including the installation of a new delay stage for the UV line and a new pump laser for our regenerative amplifier that has increased our power output while also improving stability. We have also made significant upgrades to our data collection system, as new programs were necessary for both data analysis and collection.

We have taken preliminary data on model metal-carbonyls in order to evaluate the full power of T2D-IR in mapping out photochemical mechanisms. As an initial proof of principle experiment, we are studying the photochemistry of iron pentacarbonyl, which we have previously studied via both T1D-IR and 2D-IR. It is well known that UV excitation of $\mathrm{Fe}(\mathrm{CO})_{5}$ in solution results in the loss of one carbonyl, but previous efforts, by our group and others, have not been able to determine conclusively whether an equatorial or an axial carbonyl is preferentially dissociated. The spin state and geometry of $\mathrm{Fe}(\mathrm{CO})_{4}$ have also been debated; it is unclear if this species is a singlet or a triplet. Additional experimental data and DFT calculations are required before conclusions can be made for this species. In completing this experiment, we will develop methods for later mechanistic studies.

We have also completed a detailed T1D-IR study of $\mathrm{Cp} * \mathrm{~W}(\mathrm{CO})_{3}(\mathrm{Bpin})\left(\mathrm{pin}=1,2-\mathrm{O}_{2} \mathrm{C}_{2}-\right.$ $\left.\left(\mathrm{CH}_{3}\right)_{4}\right)$ in neat pentane solution on the pico- through microsecond time-scales using UV-pump, IR-probe spectroscopy and step-scan FTIR spectroscopies. Photoexcitation of the parent complex forms two isomers of the first intermediate, resulting in two possible pathways for this reaction to proceed. We anticipate that T2D-IR can give us structural information on the intermediates; by examining possible transition state structures, we can explain the differences in reactivity between them. This study has been accepted for publication in JACS. 


\section{Metal Nitrosyl Complexes and Catalytic C-H Bond Functionalization \\ Principal Investigator(s): F. Dean Toste}

\section{Project Description}

The goal of the research described in this proposal is to uncover new reactions of organic compounds with homogeneous transition metal complexes and obtain a basic mechanistic understanding of these transformations. The direct functionalization of carbon-hydrogen bonds to form reactive carbon-metal that can undergo further transformations has been the focus of intense effort. While significant advances have been made in the activation of aromatic $\mathrm{C}-\mathrm{H}$ bonds, in stark contrast, considerably less progress has been made towards the selective activation of olefinic $\mathrm{C}-\mathrm{H}$ bonds. Given that alpha-olefins are a common chemical feedstock and important intermediates in fine chemical synthesis, we aim to develop a catalytic process for the functionalization of vinylic $\mathrm{C}-\mathrm{H}$ bonds.

Our approach relies on rational organic and organometallic synthesis to propose and develop a catalytic cycle for the functionalization on olefinic $\mathrm{C}-\mathrm{H}$ bonds. In this endeavor we will (1) study and develop reactions for formation and retrocycloaddition of cobalt dinitrosyl/alkene and (2) explore dinitrosyl complexes bearing other metals and ligands in analogous chemistry. Understanding the underlying reactivity of the individuals steps will not only expand the fundamental chemical understanding of metalorganic interactions, but will be applicable to more complex molecular systems, and aid in the development of new practical applications for synthesis.

Accomplishments

We have accomplished all of the stoichiometric reactions required for the proposed catalysis. The reactions shown below for the $\mathrm{C}-\mathrm{H}$ functionalization of cyclopentene is illustrative. We initially prepared of a variety of stable, isolable cobalt dinitrosoalkane complexes and found that electron deficient olefins (i.e. Michael acceptors) react with the deprotonated dinitrosoalkane in the presence of lanthanide-based Lewis acids (eq. 1). We have also discovered that less sterically hindered cobalt dinitrosoalkanes undergo a tandem conjugate addition-intramolecular cyclization onto the carbonyl group of the conjugate acceptor (eq 2). After retrocycloaddition these sequence provides access to tri- and tetrasubstituted alenes from disubstituted alkenes through two $\mathrm{C}-\mathrm{H}$ functionalization reactions. The unusual architectures that can be accessed with this double addition chemistry, coupled with the presence of the vicinal amine moiety, might allow for the synthesis of several interesting new amine ligands for metal catalysis. We are currently developing the catalytic and enantioselective variants of these transformations.
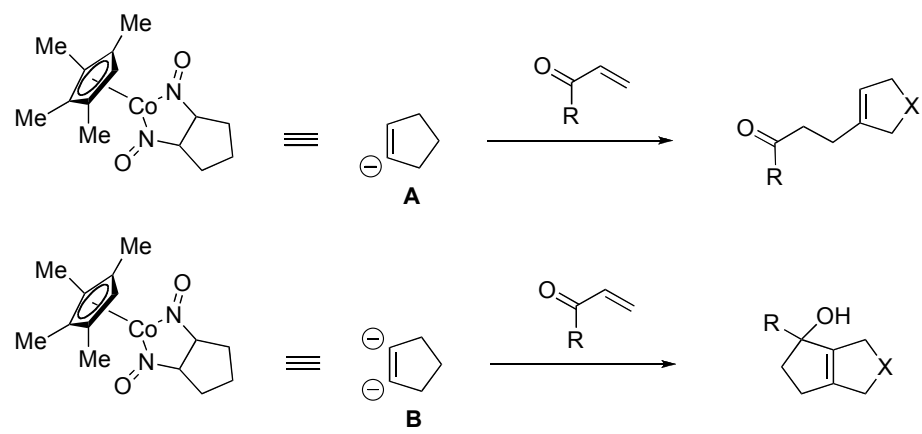


\section{Computing Sciences}

A Structured, Adaptive Mesh Refinement Method for Multiphase Reactive Transport in Groundwater

Principle Investigator: John B. Bell

\section{Project Description}

High-fidelity simulations of groundwater flow have the potential for providing valuable insights into the long-term fate of contaminants. However, realizing this potential presents significant computational challenges. We propose to perform a proof of concept study to demonstrate the effectiveness of adaptive mesh refinement (AMR) for reactive subsurface transport. The project will develop a basic flow module for modeling multiphase, multicomponent non-isothermal flow with reactions. This methodology will include second-order discretization methods that provide a robust and accurate treatment of transport processes and the coupling of that integration scheme to parallel AMR methodology. The overall goal is to validate and demonstrate the effectiveness of this approach.

The flow of subsurface fluids is governed by conservation laws for chemical species and energy, including the effects of geochemistry and phase behavior, together with phase velocities given by Darcy's law. Our approach is based on a sequential formulation of the porous media flow equations. In this approach we first solve a pressure equation. We then solve the multicomponent conservation and energy equations using a fractional flow formulation. The overall integration approach is combined with AMR methods that include support for large distributed multiprocessors. Specialized linear solver must also be developed to address the anisotropic meshes typically used for subsurface flow problems.

Accomplishments

We have made three significant accomplishments in this project this year. First, we have used our methodology to assess the role of mixing and gravity driven convection in carbon sequestration problem, in collaboration with colleagues from Earth

Sciences Division at LBL. We performed high-resolution simulations to characterize the impact of diffusion-convection processes on the storage of carbon dioxide in saline aquifers. Simulation results showed that the empirical relations between the onset time of convection and rock properties are similar to those derived from linear stability analysis. The results also revealed that the long-term behavior of the process is predominantly convective, allowing us to propose a simplified model that can be efficiently deployed in a reservoir-scale simulation. Finally, detailed 3D simulations revealed nontrivial convective patterns that cannot be readily deduced from 2D simulations.

The second significant accomplishment involves our continual efforts in incorporating realistic geochemistry into the algorithm. We improved the robustness of the algorithm and tested our approach on different types of geological problems, such as the reactive saltdome problem. The third significant accomplishment is the incorporation of geostatistical methods. The permeability and porosity fields can now be generated using established geostatistical techniques adapted from GSTAT package. We have also developed a suite of post-processing tools, allowing us to perform statistical analyses on the results obtained from the AMR simulations. 
Low Order Models for Simulation of Ballistic Charge Transport in Nanoscale Devices

Principle Investigator: John B. Bell

\section{Project Description}

As size of electronic devices shrinks to nanometer scale, ballistic charge transport becomes increasingly important in describing the transport phenomena in these devices. However, its simulation is usually computationally intensive due to the large number of states required to accurately determine the distribution of the electron density and the number of self-consistent iterations needed to achieve convergence. The project will develop a computational strategy that utilizes model-order reduction techniques. The goal is to validate and demonstrate the effectiveness of the resulting approach.

Our formulation for the ballistic charge transport is based on the Schröedinger Poisson system of equations that must be solved self-consistently. Our proposed approach integrates the reduced basis method, a model-order reduction technique that exploits dimension reduction afforded by the smooth and low-dimensional parametrically induced solution manifold, with the subband decomposition method. We also exploit an a posteriori error estimation procedure to systematically control the accuracy of our solutions. We will initially examine its application to a double-gate nano-MOSFET device. We anticipate its greater utility in the rapid design of nanotubes-based devices.

\section{Accomplishments}

We have made two significant accomplishments. First, we have extended the approach to a three-dimensional silicon nanowire-based MOSFET. In particular, we have improved the efficiency of the MATLAB codes and parallelized the codes to utilize the new parallel feature of MATLAB, allowing the larger algebraic system resulting from a 3D discretization to be solved more efficiently. We have also devised a method to handle the degeneracy of the solutions to the eigenproblem in higher spatial dimensions within the reduced basis framework. We are still in the process of validating results from the current code with existing results.

Second, we have improved the basic algorithm for solving the coupled nonlinear system of equations. Instead of using the fixed-point algorithm that has poor convergence behavior, we showed that the use of conjugate-gradient based method leads to better convergence property, enabling us to solve 3D problems more efficiently. We have also shown that a physics-based preconditioner is necessary for the conjugategradient based method to work properly due to the nonlocal coupling between the equations. 
Enhancing the Effectiveness of Manycore Chip Technologies for High-End Computing Principal Investigators: Jonathan Carter, Leonid Oliker, and John Shalf

Project Description

The goal of this project is to investigate the key challenge for next-generation highend computing systems - effectively utilizing computing systems with exponentially increasing numbers of cores per chip. Recent evidence points to the fact that future petascale computing platforms will be constructed from chips containing an increasing number of cores. It also points to a convergence towards manycore designs that may include hundreds or even thousands of cores.

Our work examines a broad range of manycore systems from small loosely-connected systems to tightly-coupled supercomputers, exploring both heterogeneous and homogeneous designs. We have started to evaluate single-socket manycore architectures across a key set of algorithms using various application probes. Additionally, we have conducted studies on large-scale manycore systems, to explore the effect of these technologies on candidate petascale DOE applications. Further, since power is becoming much more of an issue with deploying high-end computers, power consumption for these technologies has also been considered as a key metric.

These advances can be transferred to the broader scientific community to improve the efficiency of simulations. In addition, our results will provide a basis to engage and influence system designers and the computational community. Finally, our work will contribute to LBNL's role of defining and fielding the most effective computing technology for large scale scientific computing.

Accomplishments

Our recent study using a Lattice-Boltzmann magnetohydrodynamics application has shown that the auto-tuning techniques we have developed for single-nodes can be leveraged to produce optimal code for large-scale multi-node systems. In this study, we not only tune to find the best parameters for cache-blocking factors, loop-unrolling, etc., but also to find the optimal way to break up the computational grid amongst multiple nodes. This may indicate a way to automatically overcome bottlenecks that represent a major challenge to improving the scaling of many simulation codes.

Turning to our second application area of interest, we have designed a more complete stencil microbenchmark, which encapsulates the essential features of many higher-order stencil computations, and ported and optimized it for a wide range of manycore architectures, including graphical processing units. Scientific applications in many diverse areas are implemented using the stencil technique, where a sweep over a spatial grid performing nearest neighbor computation is performed. The results from this study, showing substantial performance improvements, have been presented at an international forum, and accepted for publication in a major book on the autotuning for multicore.

In our final application area, we composed new parallelization strategies for the particle-to-grid interpolation step in the Gyrokinetic Toroidal Code (GTC), a 3D particlein-cell (PIC) application to study turbulent transport in magnetic-confinement fusion devices. We found that our best strategies were $2 \mathrm{X}$ faster than the reference implementation, and our analysis provided insight into desirable architectural features for high-performance PIC simulation codes. 
On-Demand Overlays for Scientific Applications

Principal Investigator(s): Deb Agarwal, Chin Guok

\section{Project Description}

Scientific computations and collaborations increasingly rely on the network to provide high-speed data transfer, disseminate results, access instruments, support computational steering, etc. Networks now enable the reservation of dedicated high-speed paths. Utilization of these dedicated paths enables applications to achieve significantly higher bandwidth and lower latency for their transfers. However, the technique of manually configuring the application to request and utilize on-demand high-speed paths is time consuming, does not scale, and is error prone. Ideally algorithms to effectively allocate and utilize dedicated high-speed paths should be able to automatically reroute application traffic. This capability is required for an important application class within the DOE scientific community: large point-to-point file transfers. The overall goal of this project is to perform the research and development necessary to create proof-of-concept on-demand overlays for scientific applications that make efficient and effective use of the available network resources

Application overlay networks have been effective in providing data aggregation and dissemination services in the general Internet. An application overlay network creates a virtual application-layer topology that sits on top of the physical network. This application-layer topology can be dynamically configured either through a centralized decision point or a distributed algorithm. This enables the application overlays to optimize network utilization for the application data traffic. This is in contrast to current Shortest Path First (SPF) approaches that always choose a single "best" path. The majority of overlay algorithms and protocols have been designed to work at the application layer of the networking stack. We believe that the existing work at the application layer can be applied to produce algorithms and protocols that make efficient and effective use of resources at lower layers of the network stack.

Accomplishments

In this project, we developed a prototype system that can transparently route application traffic onto an overlay network on ESnet. The system uses the Phoebus software from the University of Delaware and the OSCARS dynamic circuit reservation capability. The result is that an application can simply begin transferring data and the system automatically instantiates an on-demand high-speed virtual overlay on the physical ESnet network and routes the traffic over the new virtual circuit. This new routing mechanism allows users to take full advantage of the on-demand overlay network without needing to understand the reservation and routing process. In this past year, we completed the prototype and conducted an extensive range of tests to understand the effectiveness and limitations of the resulting bulk data transfer capability. One important result from these tests was an understanding that using an overlay network allows for segmentation of the network path into local-area and dedicated wide-area segments and enables significantly improved data transfer performance. Our work has resulted in two proposals that were submitted to DOE, and we have a paper accepted for publication at CCGrid 2010 describing the research undertaken in this project. This paper, in addition to describing the research, summarizes our testing results. 


\title{
Energy Smart Disk-Based Mass Storage System
}

\author{
Principal Investigator(s): Doron Rotem, Ekow Otoo
}

\section{Project Description}

The Energy Smart Disk-Based Mass Storage System project addresses a software approach for implementing energy efficient storage systems. The primary goal is to investigate, implement and deploy modules for the management of reliable low-cost disk-based storage systems that maximize the energy savings while meeting the performance goals of the storage systems with respect to access latency, reliability and operational costs. The main ideas involve: i) utilizing the concepts of a massive array of idle disks (MAID) where idle disks are powered down when not in use; ii) consolidating resource use in both time and space to facilitate powering down of unused hardware components; and iii) implementing energy aware cache replacement policies and algorithms where emerging hybrid-disks technology, i.e., disks that are augmented with Solid State Drives (or SSDs), are deployed. The project's goals also include the development of analytical and experimental simulation models for analyzing energy consumption of different configurations of these system and configuring a prototype system that embeds our solutions.

\section{Accomplishments}

Our main accomplishments for the past year include (a) development of analytical models for power savings in data centers and (b) development of algorithms for dynamic data exchange among the disks for maximizing energy conservation. In (a) we developed a mathematical model (based on queuing theory) of the performance and power savings of disk systems given workload and disk characteristics. We applied the tool to several real life scientific application traces and showed an improvement in amount of power saving achieved as compared with existing algorithms. In (b) we developed dynamic algorithms for data exchange that can adjust to varying workloads with very small overhead. The data exchange is performed under response time constraints. Our results show that we can save up to $50 \%$ in energy consumption with minimal degradation of response time. We implemented simulation models (using the SimPy framework) for validating our dynamic data exchange algorithms. Our models have been tested with simulated and real workloads obtained from NERSC and the University of Massachusetts Trace Repository. Our work has resulted in three accepted conference papers, a fourth paper which has been submitted for publication and a fifth paper which is in preparation. As an illustrative example of this work, the chart below shows the percentage of power savings achieved by our algorithm called Dynamic Block Exchange (BLKEXG) versus other known algorithms such as DPM (Dynamic Power Management). The chart shows that BLKEXG power savings exceed that of DPM by more than a factor of 3 .

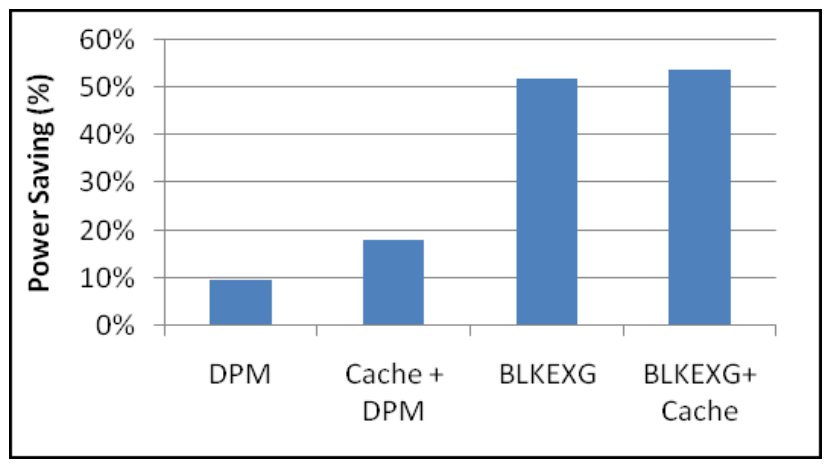

Figure 1: The percentage of power savings BLKEXG compared to the DPM method. 


\title{
A Holistic Approach to Energy Efficient Computing Architecture
}

\section{Project Description}

\author{
J. Shalf, L. Oliker, M. Wehner
}

The goal of this project is to develop new technology for energy-efficient computational science. The approach is to take a vertical slice through the space of applications, algorithms, software, and hardware to perform a study on how to build the most energy-efficient system to solve a particular computation science problem. The initial target will be climate simulation, and the solution is likely to require higher degrees of parallelism in the climate algorithms and software, as well as new hardware designs based on technology from outside the traditional workstation and server domains, particularly low-power embedded technology. The result will be a proof-of-concept machine design, along with supporting analyses of how this compares to alternative designs, and new algorithms and software techniques as needed to make the case for feasibility of computing the science problem on the system. . The project is led by John Shalf and will coordinate with other energy-efficient projects, including the LDRDs by Erich Strohmaier, Jonathan Carter, and Doron Rotem.

\section{Major Milestones in 2009}

We have fully characterized a number of different formulations of the global cloud-resolving climate model (GCRM) to further refine our projected computational requirements and expanded into new areas including geophysical and seismic data processing. We have further developed the prototype processor design of a power-efficient processor design to enable simulation of a full chip design. The SC2009 conference in November of 2009 in Portland, Oregon, which was enabled by substantial progress in the areas described below.

\section{Code Analysis}

We have expanded the analysis of the climate codes to include other candidate formulations of the GCRM including hydrostatic, anelastic, and a new hybrid formulation. The characterization further refined our memory capacity and bandwidth requirements, which are a major determining factor for power consumption. We have also worked with Fraunhofer Institute to understand how our approach could be applied to energy-efficient computation for seismic imaging and geophysics applications.

\section{Hardware Design}

In early 2009 we demonstrated our energy-efficient core design running the full climate model, and were able to demonstrate that our core design is more than 100 times more energy efficient in terms of delivered climate code performance per watt than the most energy-efficient competing solution (BlueGene/P). We have expanded our study to include architectural modeling of the entire multiprocessor chip design that includes novel hardware features that enable efficient parallel programming and communication. We have submitted an invention application to LBNL's Technology Transfer office.

\section{Autotuning}

Last year we demonstrated the effectiveness of our proof-of-concept for the auto-tuning framework. We also demonstrated that the framework could be used to tune for the novel energy-efficient hardware communication features we built into the hardware design. This work was translated into a successful Math/CS proposal to further develop this work. We used the auto-tuning to demonstrate hardware/software co-design concept called 'co-tuning,' which we presented at the SC2009 conference. We demonstrated that auto-tuning combined with automated exploration of the hardware design (using our hardware emulation platform) can improve energy efficiency by an additional 3 to 4 times over conventional hardware design techniques. This is central to our assertion that a holistic approach that combines algorithm design, hardware, and software tuning together enables a substantial improvement over business-as-usual approaches to HPC system design. 
Reference Benchmarks for the Dwarfs

Principal Investigator: Erich Strohmaier, HPCRD

Project Description

We are developing a set of benchmarks representing important groups of algorithms known as 'dwarfs'. Each dwarf captures a pattern of computation and communication common for a class of important applications. Thus, each dwarf represents a class of algorithms with similar properties and behaviors. This new set of benchmarks is designed to replace existing benchmark set used in research and to serve as a focal point of research for years to come strongly influencing the future direction of the hardware and software industries. It is designed to enable research in a variety of areas such as on-chip and off-chip computer architectures, energy efficient computing, parallel programming paradigms, compilers, and system level software.

To avoid preordaining parallel codes, we are developing pencil and paper description for each algorithm, and create realistic, scalable problem descriptions and input datasets to enable experiments from single sockets up to full scale HPC systems. We are providing means to verify proper and accurate program execution and we are also developing reference implementations in high-level languages such as MATLAB to demonstrate the algorithms in a simple fashion but with no performance expectations.

To promote energy efficient computing, we are also researching new methodologies for ranking supercomputers to augment rankings such as the TOP500. This includes the use of alterative benchmarks as well as new metrics, which include effects of power and resource consumption of computer systems.

\section{Accomplishments}

We selected a representative list of more than 20 kernels for the scientific computing dwarfs and have developed reference implementations for most of these kernels in MATLAB and in some cases for simplicity reasons in $\mathrm{C}$. These implementations include procedures to generate input data sets and problem descriptions of various sizes and routines to access accurate and correct execution of implementations. We identified kernels which represent alternative computational procedures to solve the same scientific problem. We use these commonalities for defining shared problem description, which ultimately will permit cross-comparisons even between different dwarfs. Our implementations are now being tested by outside collaborators.

One fundamental design criteria for our reference benchmarks is that we allow enough flexibility in the implementation to allow achieving optimal performance on a diverse set of computer architectures for fair comparisons. Hardware specific optimizations of code are necessary to achieve this but are potentially very labor intensive and cumbersome. However, application programmers are facing the same optimization task. Auto- tuning techniques on the sources code level can eliminate the burden associated with such optimizations and often achieve results better than compiler or expert code developers can. We characterized previous efforts of auto-tuning according to the dwarfs and started auto-tuning projects for dwarfs previously not covered. In particular we explored time and memory saving opportunities in a plasma fusion code (GTC), which belongs to the class of the n-Body dwarf. One of the most challenging kernels in this code is the particle-to-grid interpolation step for which we demonstrated substantial potential for memory savings on multi-core processors.

To facilitate research in power consumption, we published our concept for a generalized utility metric which includes power consumption in rankings of supercomputers and we continued our engagement in this community in conferences such as SC09. 


\section{Managing Petascale Data with Emerging Computer Architectures \\ Principal Investigators: Kesheng Wu, Kamesh Madduri}

\section{Project Description:}

The goal of this research project is to investigate new parallel, memory-efficient approaches for large-scale data management and analysis. Compact data representations, exploiting concurrency and locality in computation, and a careful orchestration of data access patterns of analytics routines are crucial for processing massive datasets. By effectively harnessing emerging multicore architectures, we will design scalable tools for scientific data analysis. We will also investigate new approaches to solve combinatorial graph-theoretic problems at scale, as this class of computations is becoming increasingly important in data analysis. In addition to large data volumes, data heterogeneity, variable data quality, and temporal analysis requirements are other formidable challenges to consider in the design of scalable analytics routines. Our objective is to develop algorithms that encompass several datacentric parallel performance optimizations, and that are applicable to multiple application domains.

\section{Accomplishments:}

In scientific data analysis, many routines can be expressed as relational database joins, or can be translated into join queries. For example, in astrophysics, one might need to match the records from one astronomical observation with another; such a matching of records is a database range join. In combinatorial chemistry, one might need to compare one set of molecular configurations with another; such a comparison can be expressed as joins as well. A third example is a path-related query in chain-of-evidence type of analysis in security applications. We have designed new algorithms to speed up evaluation of ad-hoc range join queries. Our algorithms use compressed bitmap indices to represent the join output compactly, and give significant performance gains over competing approaches. For instance, our count-only algorithm is up to three orders of magnitude faster, and our best bitmap index-based algorithm is 1.2--80X faster than the commonly-used sort-merge algorithm, for various test query instances on real data sets.

In collaboration with the visualization group, we have been exploring a number of different options for accelerating the searching operations involving scientific data on multicore architectures and GPUs. We have designed a number of new data structures to take advantage of the parallel computing power offered by these new platforms. Tests show that these new parallel indexing algorithms can fully take advantage of their computing powers while staying within the memory and bandwidth limitations.

The modeling and analysis of massive, dynamically evolving semantic networks raises new and challenging research problems. Due to their large memory footprint, fine-grained computational granularity, and non-contiguous concurrent accesses to global data structures with low degrees of locality, massive graph problems pose serious challenges on current parallel machines. In recent work, we present the first study of parallel combinatorial techniques for analyzing large-scale information networks, encapsulating dynamic interaction data in the order of billions of entities. We design new data structures to represent dynamic interaction networks, and discuss algorithms for processing parallel insertions and deletions of edges in small-world networks. We also designed a new lock-free parallel algorithm for computing betweenness centrality, a key computational metric in the study of complex networks. 


\title{
Earth Sciences Division
}

\author{
Applications of Adjoint Field Methods and Time-Reversal Data Processing to \\ Inverse Problems in Electromagnetics, Seismics, and Ultrasonics \\ Principal Investigator: James G. Berryman
}

\section{Project Description}

Research in complex geophysical imaging situations has shown that one robust method of attacking many imaging and target localization problems confronting geoscientists is the adjoint field method. This method appeared early in reflection-seismic imaging for oil industry applications, in part because the enormous size of the data sets had precluded the use of any other method. This approach and its generalizations, such as time-reversal imaging, have great value in many applications including nonlinear problems in ultrasonic and electromagnetic imagin, as well as seismic imaging. For example, time-reversal data processing has been developed for diagnosis and treatment in biomedical applications and for secure submarine-to-submarine communications underwater. More traditional ultrasonic imaging methods such as Non-Destructive Evaluation (NDE) have also been incorporating these methods recently.

Two application areas pursued here are: (1) electromagnetic target location, counting, and identification in the earth, and (2) methods of focusing sound and/or seismic waves in spatial regions where increased sound pressure can result in directed motion of fluids for either biomedical (drug delivery), or oil industry applications (e.g., unsticking stuck oil underground).

Accomplishments

Studies were undertaken this year for enhancing current LBNL methods for UXO (unexploded ordnance) detection and identification with the time-reversal data processing ideas. This approach leads to a computationally useful data decomposition resulting in symmetric $3 \times 3$ complex matrices that increase data processing speeds for field applications, and may lead to advantages for hand-held (much smaller than the current version) UXO detection and identification devices. One particularly important application is the ability to count the number of UXO's present in one location with this technology. A method for focusing either ultrasound or seismic waves that results in a tool for forcing fluids to move in a user-specified direction was also been developed. One paper developing these ideas has been written, as well as a patent disclosure. The paper has been awaiting the patent process completion before submittal for publication. A patent application process was completed in 2009. The idea behind this patent is especially useful for biomedical applications where one needs (for example: in the human brain) to move drugs around the delicate areas of the body with as little physical invasion (by catheters, needles, etc.) of the targeted region (requiring the drug therapy) as possible. Similarly, among the earth sciences applications, the pertinent ones include dislodging stuck oil, and/or of forcing underground contaminants towards a collection point. Current methods used in the earth are necessarily very time and energy inefficient, as there has been no obvious way to focus the seismic waves produced by programmable surface seismic vibrators towards the underground regions of most interest. The time-reversal methods (which are based on general properties of point-source Green's functions) supply one needed version of such a procedure. 


\section{Uncovering the mechanistic basis for soil microbial community response to altered precipitation patterns. Principal Investigator: Eoin L. Brodie}

\section{Project Description}

The goal of this research is to provide a mechanistic understanding of the changes in soil microbial composition and subsequent changes in metabolic function in response to altered precipitation patterns. We propose to uncover these mechanisms using a combined approach of next-generation nucleic acid sequencing to interrogate the (meta)transcriptome of microbial isolates and communities, and precise analyses of metabolites predicted to respond to altered soil moisture (e.g. compatible solutes).

We are analyzing the response of three distinct soil microbial communities to precipitation manipulations. The three soil microbial populations to be studied (desert, Mediterranean grassland, tropical rainforest) have evolved under vastly different rainfall regimes and thus allow for an initial comparison of evolutionary constraints upon the adaptation of microbial communities to climate change.

\section{Accomplishments}

We have extracted DNA and RNA rainfall manipulations experiments from all three sites. 16S rRNA genes were amplified and PhyloChip G3 microarrays have been run for the Luquillo (Tropical Forest) rainfall exclusion experiment plus controls for the 2008 experiment. This has demonstrated that although overall bacterial community composition was not significantly different between exclusion plots and controls plots nine months after exclusion shelters were removed, certain bacterial species (e.g. Actinobacteria) exhibited a legacy effect and were of greater abundance where rainfall was excluded. Samples from the 2009 Puerto Rico rainfall exclusion experiment and the Jornada (Desert Rangeland) manipulation are currently being analyzed.

A postdoctoral researcher was hired and has begun isolating representative Actinobacteria from the Tropical Forest, Desert Rangeland and Mediterranean grassland for physiological, metabolomic and transcriptomic assays. We have developed a set of barcoded sequencing primers across the three microbial domains that are currently being tested for potential amplification biases and the capacity to provide quantitative data on community composition. We have also established a wet-lab pipeline for the isolation and amplification of isolate and environmental mRNA for (meta)transcriptomic analysis by next-generation sequencing. This will be used to determine the transcriptional response of isolates and communities to altered water potential in the coming year. 


\section{Integrated Earth Systems Climate Modeling and Analysis Principal Investigator: William Collins}

\section{Project Description}

The effects of climate-change mitigation strategies on the integrated Earth system are not well understood. The main goal of this pilot project is to integrate mitigation with modeling and observation of the climate. Studies of biofuels have usuall focused on the direct climatic effects form reduced emissions of long-lived greenhouse gases (LLGHGs). However, large-scale adoption of biofuels could also significantly reduce the amount of anthropogenic aerosols. Anthropogenic sulfate and carbonaceous species are the most important types of aerosol for cooling the climate system. The introduction of biofuels could reduce the radiative cooling by aerosols and possibly affect regional temperature, precipitation, and carbon cycle dynamics. This project will investigate the effects of introducing biofuels on the physics, chemistry, and biogeochemistry of the Earth system.

\section{Accomplishments}

The first accomplishment was aimed to development of an idealized biofuels emissions scenario. A collaboration with PNNL was established to use their latest emissions scenarios developed for the U.S. Climate Change Technology Program (CCTP). These scenarios represent projections of future emissions of greenhouse gases and climate-changing chemical species for a variety of possible carbon-reduction technologies. "Bio and CCS" scenarios in which biofuels constitute the primary mitigation strategy were compared to the new reference (no climate policy) scenarios.

Key features are:

- $\quad$ Each technology dimension has a reference and advanced set of assumptions.

- $\quad$ The scenarios are constructed by combining different technology assumption sets.

- $\quad$ Each scenario is run for no climate policy and various climate policy target levels.

Overview of the Scenarios

\begin{tabular}{|c|c|c|c|c|c|c|}
\hline Technology Dimension & Reference & Nuclear Reference & Nuclear Advanced & $\operatorname{cCS}$ & Bio and CCS & Renewables \\
\hline \multirow{12}{*}{$\begin{array}{l}\text { Transportation: Electric Vehicles } \\
\text { Transportation: Fuel Cell Vehicles } \\
\text { Transportation: Other } \\
\text { Buildings } \\
\text { Industry } \\
\text { Electricity and Hydrogen CCS } \\
\text { Dedicated Energy Crops } \\
\text { Hydrogen Production } \\
\text { Wind Power } \\
\text { Solar Power } \\
\text { Nuclear Fission } \\
\text { Geothermal }\end{array}$} & Reference & Reference & Reference & Reference & Reference & Reference \\
\hline & Reference & Reference & Reference & Reference & Reference & Reference \\
\hline & Reference & Reference & Reference & Reference & Reference & Reference \\
\hline & Reference & Reference & Reference & Reference & Reference & Reference \\
\hline & Reference & Reference & Reference & Reference & Reference & Reference \\
\hline & No CCS & No CCS & No CCS & Advanced & Advanced & No CCS \\
\hline & Reference & Reference & Reference & Reference & Advanced & Advanced \\
\hline & Reference & Reference & Reference & Reference & Reference & Reference \\
\hline & Reference & Reference & Reference & Reference & Reference & Advanced \\
\hline & Reference & Reference & Reference & Reference & Reference & Advanced \\
\hline & No New Nuclear & Reference & Advanced & No New Nuclear & No New Nuclear & No New Nuclear \\
\hline & Reference & Reference & Reference & Reference & Reference & Advanced \\
\hline
\end{tabular}

(Source: The role of wind in a climate constrained world: Analysis with the MiniCAM Model)

The second major accomplishment was introduction of a new and far more accurate and extensible treatment of atmospheric radiative processes in this project's target climate model, the Community Climate System Model (CCSM) version 4 [Iacono et al, 2008a,b; Collins et al, 2008]. The new treatment of radiation is designed to calculate the radiative effects of the major greenhouse gases on both terrestrial radiation and solar radiation. CCSM4 is the first climate model worldwide to include such a complete calculation of radiative forcing of the climate system. This new treatment was exploited to quantify the effects of biofuels on aerosol direct and indirect forcing which included a much more complete treatment of the chemical, microphysical, and optical properties of the aerosols than has been previously possible. 


\title{
Maximizing Photosynthetic Yield by Increasing Sink Strength
}

\author{
Principal Investigator: Christer Jansson
}

\section{Project description}

The ability of plants to convert solar energy into biomass is of immense importance since the very survival of both photoautotrophic and non-photoautotrophic organisms depends on this process. In addition, the efficiency of photosynthetic $\mathrm{CO}_{2}$ assimilation into storage and structural products by the plant is one of the main factors that determine the economic viability of biofuel production. The overall aim of the proposed project is to study the interactions of photosynthesis and the synthesis of storage and structural carbohydrates in cereals such as barley, sorghum and rice and in storage root crops like cassava.

\section{Accomplishments}

We have applied the 22K Barley1 GeneChip microarray to investigate diurnal gene regulation events of barley caryopses at the early maturation phase. In total 2,091 differentially expressed (DE) genes were identified and assigned to six major diurnal expression clusters on the basis of $\mathrm{k}$-means clustering and principal component analysis (PCA). Global analysis including HarvEST, Gene Ontology and MapMan annotations demonstrated that the variability within the dataset relates to genes involved in circadian regulation, major carbon metabolism, embryo development, response to abiotic stress and photosynthesis. A comparative analysis of our dataset and publicly available microarray data disclosed suborgan specific expression of almost all diurnal DE genes, with more than 350 genes specifically expressed in the pericarp, endosperm tissues or the embryo. Our data reveal the tight linkage between day-night cycles, changes in light and the supply of carbon and nitrogen. These processes include phloem unloading, embryo development and the biosynthesis of storage starch, fructans and storage proteins.

We have further investigated the role of the barley SUSIBA2 transcription factor and its rice ortholog during starch synthesis and source-sink communication. Transgenic rice plants overexpressing the SUSIBA2 ortholog have been developed and found to have an increased starch synthesis and seed yield.

We have continued our studies of the circadian control of gene expression during starch synthesis in the sorghum endosperm.

We have outlined the potential for cassava as a bioenergy crop in China.

We have demonstrated the potential for the antisense ODN inhibition technology in providing selection markers during the construction of transgenic plants, and for the development of gene herbicides. 
Microbiomics of Complex Microbial Communities in Environmental Samples

Principal Investigator(s): Professor Janet Jansson (Senior Staff Scientist, ESD)

\section{Project Description}

The purpose of this project is to understand the composition and function of complex microbial communities in environmental samples using an "omics" pipeline (microbiomics, metagenomics, metaproteomics, metametabolomics) with a focus on key microbial processes of relevance to climate change, bioremediation and human health. In most environments microorganisms exist as complex communities that of thousands of different species. It is the composition and activity of these communities that determines the impact microbes will have on ecosystems and man. Towards this aim we applied molecular methods to characterize entire microbial communities in environmental samples and describe their individual components as well as their roles in various biogeochemical processes and interactions with the environment and man.

Accomplishments

We have continued to develop and apply "omics" to investigate the structure and function of complex microbial communities. For example, in collaboration with Eddy Rubin (JGI) and Mark Waldrop (USGS) we successfully sequenced metagenomic DNA extracted from Alaskan permafrost before and after thawing. The unprecedented amounts of sequence (approximately $20 \mathrm{~Gb}$ ) demonstrated for the first time that $2^{\text {nd }}$ generation sequencing is applicable to complex soil communities. Our most significant finding was large changes in the microbial communities as the permafrost thawed; particularly for those involved in the methane cycle. This has significant implications for global warming due to the large amounts of carbon currently trapped in permafrost that can be released as methane during thaw.

In collaboration with James Tiedje (Michigan State University) as a JGI Flagship Project, we extracted DNA from prairie sites in Kansas, Wisconsin and Iowa in addition to soil cultivated with corn, wheat or switchgrass. The Midwest prairie represents the largest expanse of the world's most fertile soils, which makes it important as a reference site and for understanding the biological basis and ecosystem services of its microbial community. We have obtained 454 Titanium metagenome sequences from 8 samples and 454 pyrotag sequences from 56 samples and have identified microbial community compositions in the samples.

Another significant accomplishment was the successful extraction of community proteins from soil for the first time (in collaboration with Nathan Verberkmoes and Robert Hettich at ORNL). Several direct and indirect extraction methods were developed using Arthrobacter chlorophenolicus as a model inoculum. These data are the first to successfully obtain thousands of proteins from soil and should enable us to determine the functions of microorganisms in soil of relevance for bioremediation and terrestrial carbon cycling.

For our clinical research, we published the description of thousands of metabolites that differentiate individuals with different Crohn's disease phenotypes from healthy. In addition, we published the first description of a successful fecal transplantation to cure Clostridium difficile associated disease, in collaboration with Michael Sadowsky (University of Minnesota). 


\section{Impact of Climate Change on Soil Water Dynamics in Arid Areas \\ Principal Investigator(s): Hui-Hai Liu}

\section{Project Description}

Understanding soil-water dynamics in arid areas is critical for the management of a number of U.S. Department of Energy (DOE) sites, including the Nevada Test Site and Yucca Mountain Site in Nevada and the Waste Isolation Pilot Plant (WIPP) Site in New Mexico. The potential contaminant transport at these DOE sites is largely determined by soil-water dynamics (such as groundwater recharge). However, the impact of climate change on soil water dynamics in arid areas is not well understood at this point, because of the difficulties in accurately measuring soil-water flow processes under arid conditions and in quantifying complex interactions among climate, soil water, and vegetation in these areas.

The overall purpose of this study is to develop an innovative approach to assessing the impact of climate change on soil-water dynamics in arid areas. This approach is based on a new hypothesis: in arid areas, temporally averaged soil-water saturation will not be affected by climate change during growing seasons as a result of the naturally occurring optimization processes for vegetation growth. We will evaluate this hypothesis with data from literature and develop a stochastic modeling method based on the hypothesis.

\section{Accomplishments}

A comprehensive literature survey of measurement soil-water data has been performed to evaluate our hypothesis. The following criteria have been used to determine whether a data set is to be included in the evaluation: (1) climate must be arid or semiarid, such that soil water is the most important limiting factor for vegetation growth; (2) the artificial disturbance (such as grazing) of a site where data were collected is negligible, such that the natural optimization processes are expected to occur; (3) site information is very well documented, such that mean and threshold water saturations (the optimum saturation for vegetation growth) can be determined or estimated. Survey results are consistent with our hypothesis that the threshold saturation is approximately equal to the mean soil saturation in the root zone for these sites.

Based on the hypothesis, we developed a local-scale stochastic (probabilistic) modeling method for interaction among climate, soil water, and vegetation in arid areas. The method is semi-analytical. We have applied the method to study impact of climate change on soil water recharge in typical arid areas and found that the recharge variation is significantly buffered by changes in vegetation coverage. We also found that in addition to mean soil-water content in root zone, soil-water recharge is mainly determined by frequency of heavy rainfalls associated with climate changes.

We are in the process of investigating the relative importance of climate change on shrub expansion in arid areas based on the concept of the optimum soil-water saturation. Shrub expansion in arid areas, which has been reported worldwide, has significant ecological and economical implications. It also has an important impact on soil-water dynamics in the root zone. 


\section{Identifying and Predicting Climate Change Impacts on the Land-Based Components of the Water Cycle \\ Principal Investigators: Rohit Salve, William E. Dietrich, and Stefan Finsterle}

\section{Project Description}

Our goal through this effort is to (1) document the importance of the partially saturated fractured rock continuum (PSFRC) in regulating the hydrologic cycle and (2) determine how climate change can impact the hydrology of the PSFRC. We are using an unprecedented array of deep and shallow water-level and moisture-detection devices to document how pulses of rainwater pass through hillslopes, and how residual water is removed either through deep subsurface flow, plants, or transpiration during critical drying periods. We are also exploring the use of repeat geophysical surveys to detect moisture change in the deep subsurface. The key here is to identify and develop techniques to predict the influences related to meteorological and hydrological events, which may vary as climate may vary in the future.

The hypothesis of this proposal is that the underlying fractured rock in sloping landscapes regulates both the quantity and quality of water that becomes available to downslope ecosystems. An understanding of the physical makeup of this fractured continuum, and how water is stored and transmitted through it during and between precipitation events, will lead to improved predictive capabilities of trends in water quality and quantity, as dictated by changes in climate.

\section{Accomplishments}

We have initiated an intensive monitoring program within the 'Rivendell' watershed at the Angelo reserve in northern California. This has included high-resolution (temporal and spatial) measurements of the underlying water table at multiple locations within the watershed, and continuous measurements of saturation changes close to the surface, and periodic measurements of saturation changes in the underlying bedrock with neutron logging and electrical resistance tomography. In a first attempt to explain the distinctive moisture signals observed along the expected flow path, numerical simulations have been initiated to test various hypotheses regarding water transport from the land surface through the top soil and the fractured mudstone to the water table.

Our preliminary results suggest that the underlying fractured rock is the predominant pathway for water flow through this hillslope. In addition, the geophysical measurements along with the observed well water responses show that there is significant variability in the hydrologic properties of the fractured rock which results is a range of response patterns to individual rainfall events. Because rainfall during this season has been significantly lower than the annual average, these observations will help constrain our numerical model for conditions anticipated for climate shifts towards drier conditions. 


\section{Environmental Energy Technologies Division}

LB09003

Embedded Engineering, Construction Materials, and HVAC Components in Modular Energy

Systems Simulation

Principal Investigator: Vladimir Bazjanac

Project Description

The project has two goals: (1) to develop an understanding of principles and tools essential to the development of component based Building Energy Performance (BEP) simulation that can be instantiated and parameterized from a Building Information Model (BIM); and (2) to incorporate applicable principles of information science to the process of using BEP simulation and analysis by AECOO industry. Both will eventually lead to the delivery of trustworthy and in-time BEP estimates.

The project will also set the foundation necessary to rectify the problems of traditional BEP simulation, and make it possible to meet goals of the development of a component based tool that will facilitate the design of buildings with embedded engineering, construction materials and energy efficient components, and which will optimize the performance of energy systems in such buildings. This will be accomplished by deploying a methodology that will: a) enable true component based BEP simulation; b) enable change in how BEP simulation software is currently used; and c) enable direct interoperability with other (interoperable) software that is routinely used in industry projects.

Accomplishments

So far we examined the only open-standard data model of buildings (IFC) and developed a plan and strategy how to extend it with building controls and operation definitions. Accordingly, we extended GST/IDF Generator translator's data structure to include building operations, control and scheduling data for simulation. We also publicly demonstrated automatic translation of building geometry, construction materials, and schedules of operation contained in BIM for semi-automatic use in energy simulation.

All existing "whole building" energy performance simulation tools are conceptually "onedimensional" (i.e. one-directional): Energy transfer is always perpendicular to the surface of the object (i.e. wall, slab or partition) through which it is transmitted. For accurate simulation it is critical to define all surfaces that participate in the transfer and to define them precisely (i.e. their size and position). Such surfaces are called "space boundaries;" we identified five different types (we call them "levels") of space boundaries in the development of the GST/IDF Generator tool, each of which has to be accounted for in the definition of building geometry for any conceptually "one-dimensional" simulation tool.

We also developed 43 test cases to test the GST/IDF Generator tool. Subsequently, we used the same test cases to test the building geometry export from ArchiCAD (currently the only CAD software that can generate and export all five levels of space boundaries). We participated in the Open Geospatial Consortium's (OGC) AECOO-1 Testbed demonstrations which showed how BEP predictions can for the first time be generated in real time simultaneously with construction cost estimates. This enables real time comparison of first cost and annual operating cost for energy efficient building design alternatives. Since these public demonstrations (last March and May) other BIM authoring CAD vendors (Bentley, Nemetschek) have asked for the same test cases, as they intend to develop their own ability to generate all levels of space boundaries and export them from their CAD software. Their (and other CAD vendors') ability to correctly generate and export all space boundaries will be critical to wider and more effective use of building energy performance simulation in the buildings industry. 
Understanding the chemistry of innovative air cleaning technologies

Principal Investigator: Hugo Destaillats

\section{Project Description}

The goal of this project was to investigate the use of ozonation, photocatalytic oxidation, sorption and chemisorption treatments for the removal of volatile organic compounds (VOCs) from indoor air. Air-cleaning technologies employing these combined approaches can achieve important pollutant removal enhancements while extending the lifetime of catalysts and sorbents. Effective air cleaning devices may contribute significantly to improving the energy efficiency of buildings by reducing ventilation requirements. We intended to describe the underlying chemical basis by developing a technique for the characterization of gas-phase and sorbed reaction products and intermediate species generated in the reaction of model pollutants. This analysis would enable characterization the performance of innovative sorbents and catalysts designed using novel meso- and nanoscopically tailored materials.

\section{Accomplishments}

A small amount of additional funds were received to extend the study during FY2009. In the previous two years, we evaluated novel $\mathrm{TiO}_{2}$-clay nanocomposites synthesized and characterized specifically for this project. These materials showed a photocatalytic activity that was comparable with that of Degussa P25 (a reference material), and in one case (hectorite-based composites) a significantly larger active surface area for VOC adsorption. The photocatalytic degradation of toluene was studied in a flow reactor built in our laboratory. In this reactor various catalyst materials were deposited on the surface of Raschig rings and irradiated with a UVC lamp. Toluene was used in FY2008 as a model hydrophobic VOC to characterize the performances of the reactor and of the photocatalysts under various experimental conditions, including UV frequency of irradiation, relative humidity and catalyst film thickness. This experimental system provided a framework for comparing novel photocatalysts with commercial products under realistically low concentrations, short residence times and other reactor parameters that are typical of air cleaner devices. Despite the numerous publications on this field, we found that the fundamental information generated in our studies was lacking, and will contribute to develop more efficient air cleaners.

The research carried out during FY2009 expanded this study to consider the performance of the same photocatalytic system with another model indoor pollutant, formaldehyde, in order to evaluate the effect of experimental parameters on the removal of a hydrophilic pollutant. We observed that the extent of formaldehyde adsorption to clay materials was proportional to the effective surface area under dry air. However, in the presence of humidified air, the water surface layer accelerated the adsorption process, making it less dependent on the nature of the catalyst. We observed with formaldehyde similar trends as those observed with toluene, in terms of effect of the residence time, UV frequency and relative humidity on the reaction kinetics. 


\author{
Development of a Building Operating Platform \\ Principal Investigators: Philip Haves (LBNL), Alberto Sangiovanni Vincentelli (UC Berkeley)
}

\title{
Project Description
}

The goal of this project was to demonstrate the applicability of platform-based methods for the design of control systems in buildings. These methods involve the use of systematic procedures to generate optimum designs for control systems based on formal specifications. The potential benefits of the approach include (i) cost-optimal, 'correct by design' control systems for buildings, particularly energyefficient buildings with innovative integrated systems, and (ii) replicability and traceability of building control systems, providing a mechanism for accountability for performance between designer, installer and operator. The project was linked to the Building Informatics Environment LDRD project, which has developed the simulation capability required for design and testing of building control systems.

\section{Accomplishments}

We proposed a new design flow for building automation and control. Our synthesis procedure adopts two abstraction levels. The first abstraction level reads as input a description of the control algorithm in the form of a high level language (e.g. Simulink or Modelica) and generates a functional model in an intermediate representation, IF (interchange format). Then the functional model, written in IF, is mapped to a distributed architecture model that minimizes a cost function that includes performance and first cost metrics. After that, the second abstraction layer is able to compile the control program, written in IF, to any of the vender-dependent control languages. Finally, compilers for the existing control languages are used to generate code that runs on multiple processors and the communication interfaces among these processors are synthesized and added to the generated code.

The IF designed in the project is based on UC Berkeley's Metropolis Meta Model (MMM). This enables the design flow to integrate function models written in different languages and leverage existing (simulation, verification, synthesis, etc.) tools. Furthermore, to facilitate IF translations, we constructed a domain-specific IF library with basic functional components for HVAC control.

Communication interface synthesis is used for semantics preservation when the architecture does not support the semantics of the functional model. We applied previous work from Tripakis et al on synchronous semantics preservation to building control systems. Furthermore, we extended the method, which only worked for pure synchronous block diagrams, to timed and triggered block diagrams. As a result, stream equivalence is guaranteed when distributing such systems, while maximizing communication traffic efficiency and minimizing latency.

The synthesis flow was validated manually using a hierarchical room temperature control system. With local PID controllers and a LQR supervisory controller, the control system optimizes energy consumption while maintaining thermal comfort. We translated the control algorithm originally described in Simulink to the MMM-based IF, and then translated the IF to a LabVIEW model. We simulated both Simulink and LabVIEW models with the same virtual environment. The results showed that the two models are consistent. We also explored our idea of using multi-level IF translations for the tradeoff between complexity and accuracy in this stage. We then did experiments on mapping and communication interface synthesis on the room temperature control system. The mapped system was simulated, and compared it with the initial centralized model. The results showed that there is no difference between the mapped and centralized system when the communication interface is fully applied. 
Development of Novel Improved Capacitors for Pulse Power Applications Principal Investigators: John B. Kerr, Venkat Srinivasan and Marca M. Doeff.

The project has investigated the interfacial behavior of electrolytes at various electrode surfaces with a view to increasing the energy density of supercapacitors to a level that meets the Hydrid Electric Vehicle (HEV) goals without compromising power or lifetime. The scientific goal has been to provide understanding of the nanoscale interfacial phenomena that control the device performance and by doing so provide a basis to obtain understanding of interfaces in the supercapacitor area as well as other energy conversion devices (Dye Sensitized Solar Cells, Organic Light Emitting Cells, batteries and fuel cells). In addition to conventional capacitor electrolytes and electrodes we have investigated the performance of ionic liquids to increase safety and redox polymers, conducting titanium oxides and some intercalation-type electrodes that allow us to increase the device capacity. This work seeks to broaden the applicability of skills possessed by the battery group into other areas thereby providing the potential for a wider diversity of support for the electrochemistry group.

We have examined the behavior of standard electrolytes and ionic liquids against carbon and other electrodes such as reduced $\mathrm{TiO}_{2}$ (Ebonex) in supercapacitor devices. We heve performed standard electrochemical and chemical testing in addition to use of Molecular Foundry and NCEM facilities to examine nanoscale phenomena at the interfaces. Alteration of the electrolyte structures and the surfaces of the electrodes (chemical and physical modifications) has allowed us to explore the factors that influence the behavior. We have added complexity by the addition of redox polymers to add capacity and increase voltage window to achieve the HEV goals.

\section{Accomplishments}

Our most significant accomplishment has been to set up a capacitor testing capability which parallels the extensive battery testing capability at LBNL and to build upon our battery knowledge to apply the fundamental principals to capacitor systems. We have successfully completed all of the tasks that we set our selves for setting up the testing infrastructure and we have fabricated and tested all of the systems that we initially proposed. In addition we have been able to investigate some novel hybrid battery-capacitor systems including one with an interesting aqueous electrolyte which could be significantly safer than the conventional organic electrolyte systems. We are pursuing the investigation of ionic liquid electrolytes combined with polymer gels to understand the behavior of such systems in high rate pulses. As in all electrochemical systems the interfacial behavior is critical to the performance of the device and this continues to be the focus.

We anticipate that the project will begin to produce several significant publications during FY2010 which will provide the basis for further investigations of greater depth into super capacitors, the results of which will be translatable to other critically important energy storage and conversion devices. 


\section{China's Energy Future: Changes in Energy Intensity \\ Principal Investigator: Mark D. Levine}

Project Description

The topic of China's energy system is receiving increasing attention around the world. In many quarters, this attention has turned to alarm. Coal consumption is growing at an enormous rate, power plants are being constructed at the fastest pace in history of any nation, and greenhouse gas emissions are growing apace. One-third to one-half of the world's incremental demand for oil has come from China in the past five years.

After a long and successful experience in implementing energy efficiency (1980-2002), China had lost its ability to carry out energy efficiency to scale after 2002. There is a clear need for a broad assessment of factors that affect the Chinese energy system as a whole and that have caused the disturbing increase in energy intensity (ratio of energy demand growth to the growth of GDP, both expressed as a percentage change) in the period 2002-2005. The first year and one-half of the two-year LDRD addressed this issue.

Starting in 2006, China began an intense national effort to reduce energy intensity. The final one-half year has assessed factors - especially policies and programs - to control energy demand. This assessment will serve as a basis for future forecasts of energy demand growth in China and policies that will, if implemented, reduce this growth.

Project Accomplishments

Initial analysis of data on energy use and gross domestic project (GDP) for 2006 demonstrated the difficulty - as expected — for China to tackle the economic forces that drove energy demand increases. China was able to achieve a small reduction in energy intensity in 2006. This was a significant improvement from the performance from 2002-2005, but it was not near the goal that China had set for itself. We attribute the modest gains to the time needed to reestablish energy efficiency policies and to staff and train staff members in government offices responsible for the formulation and implementation of energy efficiency.

Analysis of data for 2007 and 2008 presented a different and (to many observers) unexpected result of very substantial decrease in energy intensity at a level consistent with the policy objectives that China established. This was surprising because the achievement required China not only to pass legislation empowering its bureaucracy to pursue energy efficiency and closure of inefficient industrial enterprises but also to implement the programs at the provincial and local level. The implementation required a substantial degree of capacity building in order to have impacts throughout the Chinese economy.

Our analysis identified a variety of ways that China has been able to mobilize staff to pursue energy efficiency aggressively. These ways included the requirement placed on the 1000 top industrial enterprises to achieve energy intensity targets along with capacity building in these industries; rigorous enforcement of building energy standards in rural areas; enforcement of meaningful automobile fuel economy standards; continued development and application of appliance efficiency standards; establishment of a fund to spur investment in energy efficiency; and incentives to promote closure of many small, inefficient industrial facilities.

Based on this detailed investigation, LBNL received funding to initiate a project that is creating scenarios of future energy demand in China. We anticipate that this funded project will enable LBNL to join the ranks of the leading research groups addressing future energy demand growth in China. 


\section{Probabilistic Optimization of Energy Systems in Buildings}

Principal Investigators: David Lorenzetti and Michael Sohn

\section{Project Description}

We will develop an algorithm for optimizing, in a probabilistic sense, the design of building energy systems. Probabilistic designs seek to optimize the expected value of a cost function (for example, the expected energy use), in the face of uncertainty in the variables that affect the problem (for example, uncertain heating loads and equipment failure rates). However, probabilistic design problems lead to a "combinatorial explosion" in both the number of designs that must be considered, and the effort needed to evaluate each design. This may push designers to artificially limit the number and scope of uncertain variables they will consider.

We will explore algorithms that manage this combinatorial complexity by: (1) incorporating expert judgement about likely solutions; (2) identifying dependencies among the design parameters; (3) systematically reducing the design space in light of results from earlier designs; and (4) producing useful partial results even if the algorithm does not run to completion. To do so, we will frame the optimization as a Bayesian updating problem, in which distributions of design parameters are updated to reflect the information gathered by evaluating candidate designs. At any given point in the process, the universe of possible designs will be represented as individual or joint distributions of design parameters. Initially, the distributions will represent expert judgment about the likely values, but as the optimization progresses, the distributions will come to reflect the actual performance of the designs tested so far.

\section{Accomplishments}

Initial work focused on defining the problem rigorously. We distinguish between design parameters (values that define some aspect of a design, such as a chiller setpoint temperature) and design variables (important quantities outside the designer's control, such as outdoor temperatures). A probabilistic design problem requires choosing the design parameters in order to optimize the expected performance in the face of uncertain design variables. The optimization itself need not be done probabilistically. However, we showed that deterministic optimization methods typically will require excessive effort to find the expected performance to any satisfactory level of accuracy.

Next we performed a number of feasibility studies, intended both to explore the problem space, and to test our assumptions about how a probabilistic optimizer should proceed.

The most important result from these numerical experiments is that a probabilistic optimizer should discover dependencies among the design parameters. Acting on their joint probabilities will significantly reduce the overall number of design evaluations, by helping separate variability due to the design variables from that due to the design parameters. This result motivates our current work, on how best to store and update probability distributions. Currently we approximate a single distribution as a cumulative-probability histogram with variable-width bins; we also are considering using balanced, weighted trees. In any event, some heirarchical representation will be needed in order to encode relationships between design parameters.

The feasibility studies also suggest that the optimizer occasionally will have to access a complete history of the candidate designs tried so far, in order to re-encode the parameter distributions. For example, to make the distribution of parameter $y$ vary with the value of parameter $x$ probably will require reevaluating all the candidate designs tested so far. 
Novel Techniques to Characterize Secondary Organic Aerosols Formed from Gas-Phase Volatile Organic Compounds Emitted from Biogenic Sources

Principal Investigator: Melissa Lunden

Project Description:

The overall goal of this research is to advance current understanding of the processes that govern secondary organic aerosol (SOA) formation in the atmosphere. SOA is formed by the condensation of low volatility oxidation products of gas phase volatile organic compounds (VOC). SOA constitutes a significant fraction of aerosol mass in the atmosphere, and thus has important impacts on climate and visibility. Biogenic SOA is thought to be a dominant fraction of total SOA, yet emission inventories of biogenic SOA for climate models are the most simplistic and uncertain of the current aerosol inventories. The specific goals of this project are: (1) To develop novel techniques to analyze the chemical composition of SOA, (2) To investigate the composition and yield of SOA formed by oxidation of important biogenic species, (3) To develop techniques to make quantification of SOA in the field simpler and more economical, and (4) To facilitate future improvements to process models of biogenic SOA formation in climate models. These models will prove crucial as the world moves towards a future that replaces fossil fuels with those derived from biogenic sources.

Accomplishments:

The approach of the project was to characterize biogenic SOA formed in chamber experiments utilizing emissions from important biogenic species. VOC gases were oxidized at a variety of atmospherically relevant conditions, and the condensed aerosol oxidation products analyzed utilizing Fourier Transform Infrared (FTIR) spectroscopy to allow for quantifiable measurement of all condensed organic compounds by chemical functionality.

The primary experimental method used to study the formation and subsequent chemical reaction of the secondary organic aerosols was a flow through aerosol reaction chamber constructed specifically for the project. The physical characteristics of the particles as a function of the extent of reaction were characterized using on-line aerosol instrumentation, resulting in highly time-resolved measurements of particle number concentration and size distribution as a function of the extent of reaction. The aerosols generated in the reactor were collected by filter for chemical analysis.

A relative humidity controlled chamber flushed with clean air was constructed that enables a stable environment to precisely condition collected filters and to measure aerosol mass by microbalance. A similar enclosure was constructed to house the FTIR to reduce atmospheric interference with sample analysis. Analyses performed on the filter samples showed aldehydes and ketones $(\mathrm{C}=\mathrm{O}$ bond $)$, alkanes, and an $\mathrm{O}-\mathrm{H}$ signal indicating alcohols. The fresh biogenic SOA spectrum differs from aged organic aerosol collected in a number of urban and rural areas around the world, which show larger fractions unsaturated $\mathrm{C}-\mathrm{H}$ groups and aromatics. An automatic filter collection and analysis system was designed and constructed, allowing for time resolved analysis as well as field implementation. 
Relating Tissue Residues to Indoor Chemical Sources in a Bayesian Framework:

Synthesis of Chemistry, Pharmacokinetics, and Biomarkers

Principle Investigator(s): Randy Maddalena, Tom McKone, and Michael Sohn

Project Description

Organic chemical residues from agriculture, industry, and consumer-products are widely found in human tissues (blood, urine, lipids, etc), which is raising concern about exposures and potential long-term health impacts. Efforts to assess human and ecosystem exposures through multiple environmental media have produced a number of useful modeling and monitoring tools/techniques. Unfortunately the indoor environment has been excluded from much of this work, particularly with respect to persistent and bioaccumulative semi-volatile organic chemicals (SVOCs). As a result, public health agencies continue to assess residential exposures based on sparse concentration data and detailed time-activity models. This approach requires extensive activity data, is awkward, full of assumptions and uncertainty, and difficult to validate or apply to emerging exposure biomarker data, particularly when product/chemical streams are rapidly evolving to meet the increased demand for high performance energy efficient building.

The overall goal of this project is to provide a more systematic understanding of the relationships among indoor contaminants, indoor chemistry, human exposure, and tissue burdens using a combined modeling and measurement strategy. Our approach is to build on a wellestablished modeling philosophy that uses physiochemical properties, chemical potential (fugacity) and mass-balance concepts to link indoor chemical fate processes to biomarkers of exposure.

Accomplishments:

An indoor chemical fate and occupant pharmacokinetics model was imbedded in an environmental mass balance model providing a platform to assess biomarker measurements. The system was used to evaluate the behavior of contaminants that were measured in urine samples from subjects in a California farm community. The assessment revealed that both the buildings and the occupants were acting as chemical receptors for substances that originated outdoors while the indoor environment acts as both an integrator and delivery system for pollutants. It was found that the chemical residence time in key reservoirs, including humans, was an important indicator of potential indoor exposures and the approach was used to identify chemicals for inclusion in future biomonitoring work. Datasets for model refinement and evaluation have been identified within the Centers for Disease Control and Prevention's (CDC) National Human Exposure Program. We identified the variables related to demographics and/or building characteristics within the CDC data that will allow us to evaluate the modeling system. 
Using New Microbial Assays to Characterize Dampness-Related Exposures:

Combining Molecular Biology with Building, Aerosol, and Health Science

Principal Investigator(s): Mark J. Mendell

\section{Project Description}

The overall goal of this project was to test a hypothesis that wet surfaces in air-conditioning (AC) systems can emit small microbial particles, not previously measured, that could cause health effects in building occupants. The specific goals were: 1) to identify microorganisms on wet AC surfaces (cooling coils and drip pans), comparing molecular assays to culture assays, to define possible sources for emitted microbial particles; 2 ) to identify any microorganisms on AC surfaces with prior known immunologic or allergic effects; 3 ) to perform cellular assays for inflammatory potential on samples from these AC surfaces; and 4) to facilitate future epidemiologic studies on health effects from damp AC surfaces and other damp indoor surfaces.

In the second year, the project planned to 1) collect additional samples from a California office building in a hot, dry summer climate and a Texas office building in a hot, moist summer climate, and 2) to compare microbiologic assessments of cooling coil fins and drainage pans using polymerase chain reaction (PCR) assays, RNA-based microarrays, culture-based assays, and cellular assays for inflammatory potential.

Accomplishments

In this project, we developed two sampling methods for AC cooling coil fins and drainage pans - using narrow flocked nylon swabs for surfaces of fins and pans, and sterile pipettes to quantitatively sample water collected at the base of fins and in drainage pans. We sampled in two AC air handling systems, in California and in Texas. We collected swab samples from both AC systems, and pipette samples in the California AC system.

Our analyses of swab and liquid samples included: Phylochip RNA microarrays to identify bacterial "observational taxonomic units" (OTUs); Polymerase Chain Reaction (CR) assays to identify fungi; culture assays to identify viable bacteria and fungi; and cellular assays for inflammatory potential using IL-8 and IL-6.

From this project we identified, as expected, more types of microorganisms using molecular assays than with cultures. (Sampling difficulties in Texas made interpretation of results from those samples difficult, so California results are provided here.) Findings from swab and liquid samples showed similar results, but liquid samples were quantifiable per volume sampled. Phylochip bacterial assays identified over 300 bacterial OTUs, including groups containing species with adverse health effects such as Actinomycetes, Streptomycetes, Pseudomonas, Thermoactinomycetes, and Mycobacteria, compared to cultures, which identified a maximum of 6 bacterial species per sample, including none of health consequence. Both types of bacterial assay identified Methylobacteria, which may cause fouling of coils and reduced energy efficiency. PCR fungal assays identified 2-6 species, including in almost all samples the Gramnegative bacterium Aureobasidium pullulans, a fungus with potentially serious respiratory effects, compared to culture assays that identified 1-4 species, including no species of health concern. Samples caused large increases in production of inflammatory cytokines. For IL-8, coil samples showed increases of up to 15 times over controls and pan samples up to 25 times; for IL-6, coil samples showed increases of up to 104 times over controls and pan samples up to 169 times. Growth of, and emissions from, identified organisms in AC systems has not yet been shown, but if documented would suggest potentially harmful, widespread exposures in buildings. 


\section{Project Description}

The primary goal of this project is to create a tool that automatically tunes building energy simulation models (such as EnergyPlus) to match energy and temperature data. This will enable creation of robust, validated energy models of commercial buildings, suitable for use in demand management, fault detection, and energy retrofit planning. Secondary goals are to demonstrate the tool using data from a real commercial building, and to facilitate the investigation of trade-offs between cost, number of sensors, model complexity, etc.

The project flowchart is shown below.

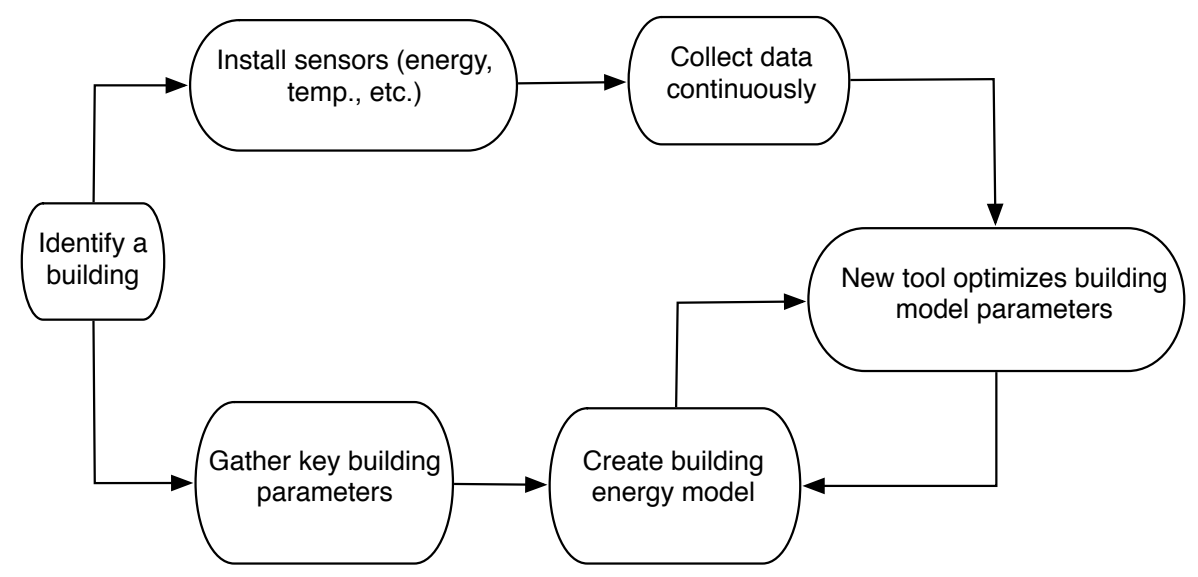

\section{Accomplishments}

Progress on the flowchart items has been made as follows:

1. Identify a building: complete (UC Merced Classroom and Office Building).

2. Install sensors: complete (building is already extensively monitored).

3. Collect sensor data: complete (data logging is ongoing).

4. Gather key building parameters: complete.

5. Create energy simulation model of building: complete (model has been rough-tuned to data).

6. Create new software tool to optimize model: in progress. Preliminary software is complete and functioning properly. Creation of this software tool is the primary goal of the project. Many improvements to the preliminary software are now being performed, including: program speedup; ability to handle data gaps; tools for graphical display of results. 
Using IP Telephony and Wireless Technologies to Extend the Reach of Conventional Building Automation Systems

Principal Investigator(s): Francis Rubinstein

\section{Project Description}

The project objective is to demonstrate the energy efficiency, demand response and comfort consequences of applying wireless communications and IP telephony to advanced building automation systems (BAS). In FY 2008, we demonstrated a proofof-concept (PoC) integrated control system for window blinds and fluorescent lights that implemented intelligent, automated control of the shading and lighting systems while allowing user overrides using energy-scavenging wireless dimmer switches. In FY 2009 we focused on extending the ways in which users can interact with the previously developed PoC system, through the accommodation of handheld personal computing devices. Two "smart phone" applications were developed and tested as described below.

\section{Accomplishments}

Our most significant accomplishment was the successful development and testing of two smart phone applications that allow the occupant and/or facility operator to easily override their lighting and shading systems using their phones. Since the target audience for the Personal Control Panel and the Demand Response application are not the same, we developed separate applications specifically designed for each intended user.

Occupant Personal Control Panel: This application allows the occupant to override the operation of an automated lighting and blind controller. This virtual control panel duplicates, rather than replaces, the functionality of the existing physical switch and dimmer. The user can override the lights and shades using the virtual control panel or the physical switches according to personal preference. Unlike the physical switch, the control panel allows the communication of useful data between the user and the controller. For example, as the user dims the lighting using the control panel, the lighting graphic on the control panel dims or brightens to provide useful feedback to the occupant about the current status of lighting and shading.

Demand Response Application: This Demand Response (DR) application allows the facility operator to trigger different DR operational modes from their smart phone when the electrical grid reaches Stage 1 (moderate) or 2 (severe) emergency condition. The DR application has information pushed to it about the current DR status as well providing the manager with the means to easily transmit the DR event status to the suitably-configured controller to effect an immediate load reduction (i.e. dimming) of the lighting system. The control panel also annunciates the particular Demand Response condition to the facility manager.

This research has positioned us to port the control algorithms and processes developed in this work to miniature wireless controllers that we believe will serve in the future as practical intelligent nodes for deploying area-based building automation services in existing buildings. 
An Inverse Approach to Characterizing Uncertain Economic Drivers of Global Climate Change Principal Investigator: Alan H. Sanstad

\section{Project Description}

Understanding the risks of global climate change and formulating effective policies to address them requires analysis of uncertainties across multiple domains. Compared to climate science and modeling, there has been relatively little formal research on uncertainties associated with the economic determinants of greenhouse gas (GHG) emissions or how to rationally account for them in policy formation. This is especially the case for "deep" uncertainties, which are those that cannot be plausibly represented in terms of probability distributions, and which are particularly critical over the long time scales needed for climate analysis. The purpose of this project is to develop and demonstrate a methodology for characterizing and analyzing deep uncertainties in economic drivers of long-run anthropogenic GHG emissions, by applying inverse techniques that are in widespread use in other disciplines but have not yet been applied in this domain. This approach is in contrast to the conventional deterministic forward "scenario" philosophy that dominates economic modeling related to climate change and GHG analysis.

\section{Accomplishments}

Our most significant accomplishment has been development of a framework that characterizes economic uncertainty in the present context within the theory of inverse or "ill-posed" problems in nonlinear algebraic systems. Numerical economic models for climate and GHG analysis are often formulated as nonlinear systems of equations representing supply and demand equilibria in multiple markets and time periods, and are solved by, e.g., Newton's Method. This approach is based on the Implicit Function Theorem, which ensures both existence and uniqueness of solutions under standard conditions. Our framework shows how deep uncertainty in parameters and other inputs in such models can be represented by enlarging the input sets to obtain an illposed nonlinear problem that contains the original as a projection or selection, when the underlying policy goal is formulated as a "target" - such as meeting a specified GHG reduction objective by a particular time. We have begun analyzing how structural features such as convexity in the underlying equation system might be used to tailor efficient solution methods for numerical implementations of this approach, particularly those based on evolutionary computation.

In addition, we investigated the use of both a genetic algorithm, and a high-dimensional Lagrange polynomial, for analyzing uncertainty in multiple-economic-model GHG emissions scenario simulations. Finally, we took first steps toward determining how the inverse approach to economic uncertainty might be combined with inverse analysis for reducing uncertainties in regional black carbon emissions estimates, for integrated modeling of anthropogenic emissions projections and climate change dynamics and impacts on a regional level. 
Software for Integrated Analysis of Sensor Data for Advanced Energy Controls Principal Investigator: Michael D. Sohn

\section{Project Description}

The purpose of this project is to develop algorithms for interpreting data that stream in from various building sensors. The resulting software will provide real-time assessment of building energy performance, occupant comfort, and fault detection. It may be applied to help design sensor networks (e.g., identifying what information is needed, and from where), tune a building's energy management and control system, flag hardware that might have become defective, and maintain optimal comfort while minimizing energy consumption. The resulting software will also allow users to compare model predictions of energy use against measurements from diverse sensors, in real time, at both the whole-building and sub-system levels.

Accomplishments

We have made significant progress in developing the conceptual and statistical algorithm for "fusing" data from multiple sensors simultaneously and in real time. Current methods for fault detection and diagnosis (FDD) in buildings are either model-based or data-driven. In general, model-based approaches signal faults when data and models differ significantly, while data-driven approaches detect faults when data from sensors report inconsistent or irregular values. Because these approaches tend to be deterministic, as opposed to probabilistic, they have difficulty identifying faults when models fit poorly, and when data are highly variable and noisy. Additionally, the model-based approaches perform many time-consuming numerical calculations, which may complicate detecting faults in real time.

This year, we confirmed and demonstrated that Bayesian updating methods can calculate the probability that a fault is occurring. We formulate a likelihood function, which is a statistical function that accounts for model-misspecification errors and data variability/uncertainty. We also use prior distributions, statistical functions that describe our belief about how the building should behave. The prior distribution allows us to incorporate our intuition in a statistically rigorous fashion.

Our research has resulted in the submission of one journal manuscript so far. The paper, "Modeling and Measurement Constraints in Fault Diagnostics for HVAC systems," discusses the conceptual approach to Bayesian fault detection. To our knowledge this paper is the first demonstration of this approach for buildings. In the paper, we examine a typical air handling unit in a large commercial building. By measuring the temperature at various locations in the HVAC system, the algorithm is able to calculate the probability that any of several conditions are occurring, such as stuck dampers, leaking ducts, and malfunctioning valves, as well as the case when no fault exists. The manuscript was submitted to the ASME Journal on Controls and Dynamic Systems, and was co-authored by M. Najafi (UCB doctoral student), D. Auslander (UCB, ME Dept.), P. Barlett (UCB CS Dept.), P. Haves (LBNL), and M. Sohn (LBNL).

We also are preparing a journal manuscript describing an approach to proactive fault diagnosis. We apply a technique called value-of-information analyses to determine what data or HVAC operation that will detect various minor operating faults before they become significant malfunctions.

Lastly, we have prepared a research proposal that outlines what is needed to test and implement our software in a real commercial building. 
Building Informatics Environment Enabling Rapid Prototyping and Model Extraction for Building Automation Systems

Principal Investigator: Michael Wetter

\section{Project Description}

The purpose of this project is to prototype a Building Informatics Environment (BIE), a software environment for modeling and simulation of building energy and control systems. The BIE will enable rapid virtual prototyping to support the invention of new building energy and control systems. It will also allow embedding physics-based models into the Building Operating Platform (BOP), which is a software environment for the design, deployment and operation of building automation systems.

Using simulation-based virtual prototyping, the BIE will enable designers to explore a larger set of design options than in a hardware test-bed. It will also allow analyzing the dynamics of integrated systems during all seasons and with different boundary conditions, and hence, designing a robust system that can subsequently be tested in a hardware test-bed to include effects that are not explicitly modeled. The BIE will enable extracting models used during design and embedding them within the BOP in order to couple them with sensor networks for commissioning, model-based controls, fault detection and diagnostics.

Accomplishments

In year 1, we developed a modular library with component models for building energy and control systems using the acausal object-oriented language Modelica. We conducted proof of concept studies for local loop controls design and simulation-based pre-commissioning of supervisory control systems.

In year 2, we demonstrated that novel whole-system models for space conditioning that are outside the capabilities of established building simulation programs can be inserted into the Building Informatics Environment significantly faster than it would take with established building simulation programs. A novel water-based heating system where water circulation pumps are located at each radiator instead of using a central pump, and where the controls is realized using distributed room-level controllers that communicate their load to a supervisory controller, has been implemented about an order of magnitude faster than it would take with established building simulation programs. We also provided a proof of concept in which airconditioning and controls models from the Building Informatics Environment are linked to the Building Controls Virtual Test Bed, a software that is currently developed at LBNL. This allows linking these models with actual building control systems for model-based operation. 


\section{LB09004}

\section{Genomics Division}

Measurement of Protein/DNA Binding in vitro and in vivo Using Single Molecule Approaches Principle Investigators: Mark Biggin and Jian Jin

\section{Project Description}

Correct modeling of the interaction of sequence specific transcription factors with DNA is critical for systems studies of complex transcriptional regulatory networks, such as those controlling animal development. We are developing novel single molecule methods to accurately measure (i) the affinity of factors to a range of binding site variants in vitro and (ii) methods to measure the relative levels of factors binding to defined genomic regions in the embryo with cellular resolution. These methods will provide a much more accurate understanding of transcription factor behavior and improve quantitative modeling.

For the in vitro assay, single protein molecules will be immobilized within a nanoarray and the dwell time and intensity of fluorescently labeled DNA oligonucleotides will be used to establish the protein/ligand affinities. A micro fluidic system will be established to allow rapid interactive measurement of multiple DNA binding site variants. The accuracy of the resulting affinity models will be benchmarked against earlier in vitro models using the relative enrichment of sites predicted by each model in genomic regions known to be bound in vivo.

For the in vivo assay, endogenous transcription factors and a specific target cis regulatory DNA region will be labeled in fixed Drosophila embryos. Using super high resolution light microscopy, the co-localization of proteins and DNA will be measured cell by cell within parts of the embryo where factor protein concentrations vary. The relative occupancy of protein on DNA will then be calculated.

Accomplishments feasibility.

We have reached important milestones on both projects, illustrating their technical

In vitro measurements. We first generated proof-of-principle single-molecule data by immobilizing transcription factor proteins on passivated glass cover slips via an antibody. By analyzing the binding/unbinding events, we showed we were able to infer absolute dissociation constants. We have also manufactured a nano-array, and established that these bind to tagged transcription factor. An automated micro fluidic device has also been built that will allow measurements to be made for a whole series of different affinity DNAs with no intervention after initial set up.

In vivo measurements. The major technical obstacles for measuring co localization of transcription factor proteins and DNA are to specifically label each with fluors suitable for single molecule detection and to detect these fluors in the embryo at high resolution. To this end, we have shown that we can use a 3D tracking microscope developed by the Yang laboratory to locate and extract super resolution localization of a labeled single protein molecule. We have also succeeded in labeling a short $(5 \mathrm{~kb})$ single copy DNA CRM in embryo nuclei and obtained excellent images. 


\section{Assessing Epigenomic Approaches for Gene Enhancer Discovery PIs: Len A. Pennacchio, Axel Visel}

\section{PROJECT DESCRIPTION}

Sequencing of the human genome, achieved with substantial contributions from DOE/LBNL/JGI, has provided a near-complete list of human genes. In sharp contrast, these efforts have identified only a marginal fraction of the regulatory sequences that play crucial roles in development and disease as they determine when and where each gene is expressed. Modest recent progress towards this goal has been made through the use of vertebrate comparative genomics. For instance, our laboratory has shown in transgenic mouse assays that $\sim 50 \%$ of extremely conserved noncoding sequences are developmental enhancers in vivo. However, despite significant effort we did not find additional sequence features that distinguish enhancers from conserved elements without enhancer activity. We propose to generate and explore genome-wide maps of enhancer-associated transcriptional co-activators in embryonic mouse tissues as a strategy to accurately identify tissue-specific enhancers. We will also determine if such epigenetic modifications exist in non-conserved DNA. Technologies developed through these efforts will represent significant achievements with strong impact on the field, as they will greatly increase our ability to identify enhancers and enable exploration of their roles in human biology and disease.

To identify sequences with chromatin modifications, we will use $\underline{\text { Chromatin-Immuno- }}$ Precipitation (ChIP) to capture nuclear DNA sequences with desired chromatin modifications. We will compare the chromatin marks present at $>1000$ enhancer candidates that we characterized in vivo in earlier studies, as well as first ChIP-seq datasets generated during the first year of this LDRD, to determine the most sensitive chromatin signatures associated with active enhancers. We will then perform transgenic mouse assays on DNA fragments with desired epigenetic features to assess their predictive power for enhancer activity. These studies will enable genome-wide identification of embryonic enhancers and facilitate their functional characterization in transgenic mice.

\section{ACCOMPLISHMENTS}

We are exploring the use of ChIP-seq mapping of chromatin marks in a variety of embryonic and adult mouse tissues and evaluated the potential of antibodies directed against additional chromatin marks, such as acetylated CBP/p300 and histone methylation marks, for the discovery of distant-acting enhancers.

We also undertook an in-depth study comparing the evolutionary conservation characteristics of p300 binding sites in different tissues. This study was enabled by the methods developed in year 1 of the LDRD funding and provided important insights into the limitations of conservationbased approaches for enhancer discovery and provides an important rationale for further expansion of ChIP-seq based approaches for enhancer discovery. A paper describing the results from this study has been submitted.

Dr. Visel obtained a grant from NIDCR (1U01DE020060-01) based on preliminary data obtained through LDRD funding. 


\section{Life Sciences Division}

Functional Interactomics: Integrating Physical and Functional Interaction Networks Principal Investigator: Gareth Butland

\section{Project Description}

Interactions, both physical and genetic define the organization of the cell. Physical interactions, such as protein-protein interactions, help us understand how individual polypeptide chains come together to form protein complexes which perform many of the biochemical reactions in a cell. Other types of interactions, such as functional interactions, propose organizational relationships between gene products and protein complexes within the cell. Epistatic (genetic) interactions, one of the most commonly encountered forms of functional interaction, exist between genes which encode proteins involved in parallel pathways or processes. These interactions, whilst not physical in nature, provide information regarding functional redundancy within the cell and help understand the basis of suppressor mutations and synthetic lethality. This LDRD proposal was designed to develop and implement a methodology capable of screening for functional interactions in E. coli on a genome wide scale.

Classical genetic approaches have often combined null alleles in the same strain to assess if a functional relationship exists between two or more genes of interest. These experiments have however usually been restricted to specific biological niches in order to limit the number of null mutations that have to be made sequentially by laborious methods. This proposal will utilize the recent availability of a complete set of $\sim 4000$ single gene knock-out (KO) strains of $E$. coli. A null mutation in a gene whose genetic interactions are to be screened will then be combined in a pair wise manner with all 4000 mutant strains present in the KO collection and the viability of the strain bearing both mutations assayed. The key step of quickly and easily combining null mutations between strains of E. coli in a manner amenable to high-throughput analysis will be developed based on the natural genetic system of conjugation. This will facilitate the rapid screening of thousands of potential functional interactions in a rapid and efficient manner.

\section{Accomplishments}

Proof-of-principle work describing a high throughput genetic interactions screening approach was published last year in Nature Methods. This study, which focused on the essential process of iron sulfur cluster biosynthesis, identified several novel genetic interactions, such as the synthetic lethality of Isc pathway mutants in combination with the monothiol glutaredoxin GrxD, implicating it for the first time as having a key role in bacterial FeS cluster biosynthesis.

Over the last year, we have continued to focus on elucidating the role of monothiol glutaredoxins in prokaryotes using a combination of high and low throughput genetic interaction and protein-protein interaction screening. This interaction data has been supplemented with more traditional complementation analysis, in vitro biochemistry and UV-Vis and EPR spectroscopy. This work has formed the basis of an application for follow-on funding in the form of an RO1 Research Project grant from the NIH. This preliminary work detailing the characterization of GrxD is currently being prepared for publication and it is our hope that two reports detailing both the physical properties of cofactor and protein partner binding by GrxD and the functional role of GrxD in the cell will be forthcoming within the next year. 
Linking genomics, proteomics and ultrastructural characterization of microbial communities and their viruses

Principal Investigator: Luis R. Comolli

\section{Project Description}

The goal is to establish and validate capabilities needed to develop a new field defined by the integration of molecular-scale biological and ultrastructural information for cultivation-independent analysis of natural microbial communities. Subsequently, we envisage a major initiative in which this approach is applied to consortia of DOE and $\mathrm{NIH}$ interest, including communities involved in bioenergy and bioremediation and human health. In a first stage we proposed to use cryo-electron tomographic (cryo-ET) methods to test hypotheses about biofilm establishment, organization, and dynamics that stem from community proteogenomic analyses of a model system: the multispecies chemoautotrophic microbial biofilms from the acid mine drainage (AMD) system within the Richmond Mine, Redding CA. We also proposed the development of a methodology for the identification of all the species in the biofilms by correlated cryo-electron microscopy (cryo-EM) and Fluorescence In-Situ Hybridization (FISH). In a second stage we will focus on the development of cell-surface specific probes for selected species in the biofilms based on proteomics and genomics data.

\section{Accomplishments}

The main scientific accomplishment is the coupling of near complete reconstruction of the genomes for three, deeply-branched, uncultivated lineages of Euryarchaea from AMD communities with 3D cryo-ET reconstruction-based analysis of cell features and community interactions. A small number of these reconstructions clearly show penetration of the cell walls and cytoplasmic membranes of nanoarchaea, designated as ARMAN, by single membrane bounded protuberances from cells of the archaeal order Thermoplasmatales. These features, along with many genes previously only affiliated with Crenarchaea or Bacteria, illustrate novel biology in organisms branching near the Crenarchaea-Euryarchaea split.

Due to limitations in current methods, the primary uncertainty in the analysis is the identification of the specific organisms involved in the document associations. This obstacle motivated the main methodological accomplishment in the work. Specifically, we have developed a protocol for correlative cryo-EM with Fluorescence In-Situ Hybridization (FISH). We are currently optimizing and refining this protocol and expect to start working on the second stage described above: development of cell-surface specific probes for selected species in the biofilms based on proteomics and genomics data. We anticipate that the ability to correlate ultrastructural and metagenomic information in community samples will be widely applicable and should open the way for ground breaking new insights in microbial ecology and biology. 
Interaction of Fragile X Mental Retardation Protein with Thymine-DNA Glycosylase: Implications in the Molecular Mechanism of Fragile X Syndrome Principal Investigator(s): Bo Hang

Project Description

Fragile X Syndrome (FXS), the most common cause of inherited mental retardation, is caused by a CGG repeat expansion in the FMR1 gene followed by hypermethylation in the FMR1 promoter, leading to pathogenic FMR1 silencing and loss of expression of the Fragile $\mathrm{X}$ Mental Retardation Protein (FMRP). The molecular mechanism by which this local epigenetic aberration is established and maintained has remained mysterious. A major issue in understanding epigenetic gene regulation has been the question of whether 5-meC at $\mathrm{CpG}$ sites in promoter regions is actively removed from vertebrate DNA. Emerging evidence has suggested that such a function may be carried out by combined action of a cytosine deaminase and a T:GDNA glycosylase such as TDG. Our initial finding that FMRP physically interacts with TDG and stimulates its T:G-DNA glycosylase activity makes TDG a candidate for participating in active demethylation at the FMR1 promoter.

The purpose of this project is to test the hypothesis that TDG plays a critical role in demethylation of the FMR1 promoter and the missing FMRP in FX cells causes an altered activity or failed targeting of TDG, thus contributing to the maintenance of hypermethylated FMR1 promoter. More generally, the data obtained will aid in understanding cellular mechanisms for transcriptional silencing by active DNA demethylation, which is currently poorly understood.

Accomplishments

Over the last year, in collaboration with the Priscilla Cooper laboratory, we have made several important findings that strengthen our original hypothesis. Using chromatin immunoprecipitation (ChIP) and re-ChIP assays, we demonstrated that TDG and FMRP are colocalized to the FMR1 promoter region in vivo, which supports our proposed mechanism of targeting TDG to the FMR1 promoter, in complex with FMRP. Further studies will be carried out to map the exact binding sites for the two proteins and to show whether TDG localization is lost in FX cells as a result of missing FMRP.

Our preliminary experiments using FX cells over-expressing FMRP showed reversal of FMR1 promoter hypermethylation in a significant fraction of cells. Combined with the data on co-localization of FMRP and TDG to this promoter, FMRP may facilitate the expression of its own gene by participating in an active demethylation process mediated by a TDG/FMRPassociated protein complex. These results provide strong support for a critical component of our hypothesis for an FMRP-mediated "feed-forward" regulatory mechanism.

Based upon our findings of novel XPG-TDG and FMRP-p68 RNA helicase interactions and gel filtration studies showing that TDG migrates in the megaDalton range with XPG and its known partners, we proposed that FMRP and TDG, together with XPG, are components of a large protein complex that has a critical function in active DNA demethylation. The mammalian XPG protein has roles in multiple DNA repair processes and has been implicated in active DNA demethylation. Proving the existence of such a complex in the cell would be a milestone step towards the understanding of active demethylation in mammalian cells. 


\title{
Genome Organizer in Carcinogenesis
}

\author{
Principal Investigator: Terumi Kohwi-Shigematsu (P.I.), Mina J. Bissell, Judith Campisi, \\ Yoshinori Kohwi, Sharon Krauss
}

Project description

The genome organizer SATB1 (Sppecial AT-rich Binding protein) is a key determinant for breast cancer metastasis. Once it is expressed during breast cancer progression, it regulates expression of $\sim 1000$ genes and alters epigenetic status of its direct target genes to promote metastasis. SATB1 expression correlates with poor breast cancer prognosis $(\mathrm{P}<0.0001)$. The purpose of this project is to study the regulation of SATB1 in breast cancer cells, how normal and immortalized breast epithelial cells respond to genetically engineered SATB1 expression, the mechanisms for these responses, and also the role of SATB1 in the cancer cell mitotic machinery. These data will provide a greater understanding of the biogenesis of breast cancer, which will help enable design of therapeutic interventions.

We will employ a series of human breast cancer lines at various stages of disease progression, immortalized non-turmorigenic lines, and normal human epithelial cells, in order to analyze the effect of SATB1 expression on gene expression, signaling events, localization of proteins, and the integrity of cell division machinery. Additionally, we will perform animal model experiments using nude mice. For our investigations, we will be using state-of-the art biochemistry, molecular biology, and microscopic imaging techniques.

Accomplishments

During the first year of funding, we found that SATB1 overexpression disrupts the mitotic apparatus of breast cancer cells. During the second year, we completed in vitro analyses for this unexpected effect of SATB1, and found that SATB1 overexpression in non-aggressive breast cancer cells causes centrosome amplification and mitotic defects (approx. 20\% or 40\%, depending upon whether immortalized or non-aggressive cancer cells), while deleting SATB1 from aggressive breast cancer cells reverses these phenotypes of mitotic defects. Furthermore, we addressed whether SATB1's capacity to impair mitotic activity is due to SATB1-dependent expression of genes that are involved in mitotic function, or whether instead it is a direct effect of SATB1 protein in mitotic cells. We have made a series of point mutations in SATB1 protein to disrupt its function in gene regulation, and examined cells expressing each of these mutants by in vitro invasion assay and cell growth assay. Based on our data, there seems to exist a distinct pathway for SATB1 promoting cancer progression, not necessarily attributed to its gene regulation capacity. In vivo studies using these mutants with nude mice for studying tumor growth are underway.

During the previous year, we also explored mechanisms underlying SATB1 activation, in non-aggressive breast cancer cells in culture. We employed a co-cell cultured strategy to find whether non-aggressive (non-SATB1 expressing) cells can activate SATB1. We found that under certain circumstances this was possible, and we confirmed the results in vivo using nude mice. These experiments show that a certain extracellular environment can trigger SATB1 activation. We still need to identify which specific factors are responsible for SATB1 activation. 


\section{A Fluorescence Energy Transfer Methodology for Visualization of \\ Simultaneous and Reversible Interactions (SPARKLE) \\ Principle Investigator: Cynthia T. McMurray}

\section{PROJECT DESCRIPTION: SPARKLE}

The purpose of SPARKLE is to monitor cellular kinetics in complex organisms. By labeling target proteins with excitable fluorophores, we can take advantage of the discreet emissions from quantum dots or suitable nancorystals (NCs) to excite acceptor partners by Fluorescence energy transfer (FRET). We intend to monitor cellular "kinetics" in 4D. The handing off of targets to different partners at distinct subcellular compartments can be monitored with time. The project overall will dramatically increase our ability to understand how multi-component complexes assemble in order to carry out their biological function upon receiving regulatory stimuli. The approach is general and can be applied to many systems. The initial goal is to understand complex intracellular events that ensue in control and disease cells. In later stages, we intend to apply the method to microorganisms in order to understand what types of cellular events can stimulate carbon capture and better harness bioenergetics.

\section{ACCOMPLISHMENTS}

We have been testing approaches to conjugation and cellular uptake of nanoctrystals (NCs) in collaboration with the Molecular Foundry scientists. In a typical preparation, we synthesized $\mathrm{RE}$ oleates from inexpensive hydrates of the corresponding chloride or nitrate salts. We then decomposed them at high temperature in a controlled manner using varying amounts of oleylamine in a mixture of coordinating and non-coordinating solvents. We determined that NC size and morphology were a function of reaction time and oleylamine stoichiometry. NCs were prepared in sizes ranging from $2-100 \mathrm{~nm}$. We were also able to prepare doped NCs, with 20 mol\% ytterbium and 2-mol\% erbium, which were used for upconverting photoluminescence (converting IR absorption to visible emission). Thus, cellular internalization can be readily monitored by fluorescence microscopy. Given these promising initial results, NCs with defined sizes can now be standardized using WANDA, the Molecular Foundry's one-of-a-kind automated parallel synthesis robot. These results not only indicated the potential for WANDA to improve upon single composition and doped NCs; but also that core-shell architectures are now possible, pointing to new nanomaterials with improved potential for imaging. We have demonstrated that $\mathrm{NCs}$ used in vitro and in vivo must be suitably passivated for aqueous solubility. As synthesized, our NCs are passivated with oleic acid and oleylamine and are only soluble in organic media. For aqueous transfer of NCs, we employed a ligand exchange process whereby the nonpolar residual passivating ligands were exchanged for higher affinity, multivalent polysaccharides bearing carboxylate ligands. For example, we were able to obtain NCs passivated with carboxymethyl-dextran (CM-dextran), a well-known non-toxic FDAapproved polymer for in vivo work. The CM-dextran offers a wide range of useful chemical and biological properties and CM-dextrans stabilize the folded (active) conformation of bound proteins. The ability for CM-dextran to stabilize folding of sensitive biopolymers may be an important consideration for preserving accurate donor and acceptor protein conformations upon conjugation. We developed several novel techniques for the chemoselective ligation of biomolecules, and successful conjugation of CM-dextran to semi-synthetic biomacromolecules. 


\section{Bio Energy Technologies and Science Integrated Efficiently (BETSIE) Principal Investigator: John A. Tainer}

\section{Project Description}

The growing energy crisis is conceivably the single biggest problem facing our society today and will certainly remain such over the next few decades. BETSIE seeks to exploit naturally occurring bio-systems for efficient carbon cycling and "photons-to-biofuels" conversions. Despite the enormous promise of biology to provide efficient energy conversion technologies, these systems have not been understood in sufficient detail at the molecular level to usefully harness their potential. BETSIE has three fundamental working hypotheses. First, we propose that energy conversion processes are governed by integrated pathways chosen, controlled, and coordinated by the same types of molecular controls that we have helped discover and characterize the molecular interactions and assemblies that protect the cell from cancer. These controls include dynamic macromolecular assemblies, handoffs, conformational switches, and disorder-to-order transitions. Second, we hypothesize that a complete knowledge of these pathways, interactions, assemblies, and conformations will allow us to re-design these pathways in predetermined ways with advantages for our energy needs. Third, we hypothesize that characterizations of these pathways as tested by redesign experiments will allow us to transplant functional pathways into organisms as needed for efficient energy production. We aim to use biochemical and structural characterizations to understand the molecular interactions and controls that are critical for carbon cycling and photon-to-fuel conversions.

\section{Accomplishments}

We have pioneered methods to structurally characterize oxygen-sensitive proteins that are important for bioenergy by Small Angle X-ray Scattering (SAXS). We have anaerobically screened 60 conditions and collected SAXS data to determine optimal conditions and multimeric states of proteins involved in hydrogen production.

We have also piloted both proteomic and metabolomic analysis of the stress response of microalga to growth in nitrogen-free media. Using a mass spectrometry approach, we identified a number of proteins are only detected in the nitrogen-starved algae, including several that are involved in carotenoid metabolism. Furthermore, by directly measuring metabolic products in these cells, we have determined that there is a striking decrease in cellular carbohydrate levels with a simultaneous increase in lipid levels, reflecting an overall shift in storage of reduced carbon as sugars to lipids (Figure 1).

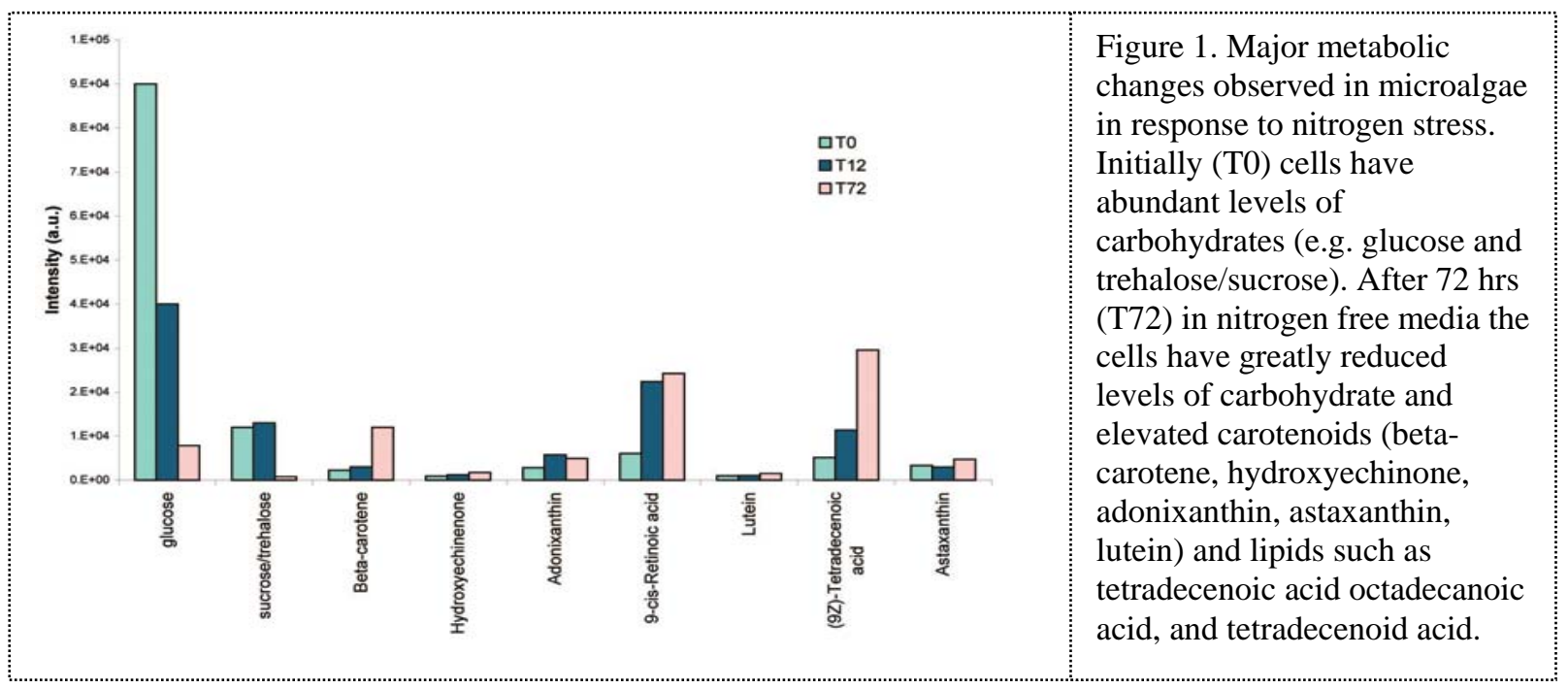


Expression Profiling of Radiation and Cancer Susceptibility Genes

Principal Investigator(s): Andrew J. Wyrobek and Francesco Marchetti

\section{Project Description}

Our objectives were to evaluate transcriptome profiles using bioinformatics tools to identify and characterize the signaling pathways that determine susceptibility for radiation-induced cancer, specifically breast cancer. It is well known that mammalian tissues differ dramatically in their sensitivity to ionizing radiation and that there are strong genetic determinants of breast cancer risk. In the first phase of this project, we characterized the expression profiles of low- and high-dose radiation damage responses among multiple tissues from two inbred strains (C57BL/6 and BALB/c) that differ in their genetic susceptibility to radiation-induced breast cancer. In the second phase, we investigated signaling pathways associated with two early proliferative barriers of breast carcinogenesis using the model of human mammary epithelial cells (HMEC) in culture. This project demonstrated the feasibility of expression profiling to investigate damage response signaling in tissues of irradiated individuals, and to identify pathways of critical cellular transitions through the early proliferative barriers to breast cancer.

\section{Accomplishments}

Radiation damage response pathways in mouse tissues.

We completed the laboratory phase of the large comparative study of damage response pathways for 600 samples of mammary gland, bone marrow, thymus, peripheral blood, central nervous system, and other tissues of mice that differ in their sensitivities for radiation-induced breast cancer. In general, we found that molecular damage responses after low-dose exposures (0.5-10 cGy) vary substantially across tissues and depend on genetic background. In brain tissue, several pathways associated with cognitive function were down regulated after low-dose exposures, and neurons appear to differ in their low-dose damage responses (two manuscripts were published in 2009). The low-dose responses of mammary glands in cancer-sensitive mice appear to be associated with inflammation, metabolism, and proliferation pathways, which was not seen in resistant mice nor after high doses. We recently obtained funding from the DOE Low Dose Program to investigate the role of genetic background and tissue microenvironment on damage signaling pathways in radiation-sensitive tissues after low-dose and radioadaptive exposures to radiation, with emphasis on breast cancer and lymphoma susceptibility.

Signaling pathways in HMECs.

We also completed a profiling and bioinformatics study of signaling pathways in HMECs, the target cell in breast cancer, to identify the molecular pathways associated with epithelial transitions through two early proliferation barriers in human breast carcinogenesis: stasis and agonescence. Epithelial cells show major changes in genes associated with myoepithelial and luminal lineages as they transition through these barriers, and the expression profiles of the transitioning cells are distinct from those of senescent fibroblasts that make up the tissue microenvironment. (These collaborative findings were published in 2009). 


\section{Project Title: Self-Assembled Battery Electrodes}

PI: Nitash Balsara

Co-PIs: Andy Minor, Venkat Srinivasan

\section{Project Description}

Our goal is to create new methodologies and materials for addressing the limitations of current batteries. While current batteries are limited in energy content to about $200 \mathrm{Wh} / \mathrm{kg}$, there is no intrinsic limit on the energy density of a battery. For example, batteries with sulfur cathodes are predicted to have energy densities of $2500 \mathrm{Wh} / \mathrm{kg}$. Similarly, the power limitations of a battery depend on the efficacy of the pathways for ionic and electronic conduction within the battery. We propose to synthesize and study model battery electrodes wherein the pathways for ionic and electronic conduction are controlled on the nanometer length scale.

Functioning battery electrodes require the conducting pathways for both ions and electrons. In conventional lithium batteries, the ions are transported by a liquid electrolyte and the electrons are transported by carbon. Our objective is to synthesize a single polymer material that exhibits both ionic and electronic conductivity. Separate pathways for conducing ions and electrons would be created by the self-assembly of block copolymers. Obtaining such polymers is the first step toward the creation of self-assembled electrodes.

\section{Accomplishments}

The main accomplishment has been the successful synthesis of a diblock copolymer with one ion-containing block and one electron-conducting block. This task turned out to be much more challenging than anticipated. Initially we had targeted regioregular head-to-tail poly(3-hexlythiophene) (PHT) as the electron conducting block. We were able to make this polymer relatively easily. Unfortunately all attempts to grow an ion-conducting block were unsuccessful. We note in passing that no group has demonstrated success in synthesizing a microstructured block copolymer with ion- and electron-transporting channels. A few months ago we decided to switch to poly(pheneylene vinylene) (PPV) as the electron-conducting block. We are pleased to report that we have successfully grown a polyethylene oxide (PEO) ion-conducting block to obtain the desired polymer. We have synthesized three PPV-PEO copolymers indicating the robustness of our procedures. Characterization of the copolymers by X-ray scattering and electron microscopy has begun. We are optimistic that conductivity measurements on PPV-PEO block copolymers will begin in early 2010. 


\author{
Transport in Thin Polymer Films
}

PI: Blandine Jérôme

\title{
Project description
}

The purpose of this project is to study the transport properties of confined polymeric systems, with a special focus on the anisotropy of the diffusion through confined systems, and the effect of the conformation of polymer chains on transport. This requires developing Resonance Soft Xray Reflectivity to obtain the necessary orientational information on polymer chain conformation.

Our approach consists in studying in parallel the structure of confined polymers (developing and exploiting new possibilities offered by Resonant Soft X-ray reflectivity) and the resulting transport properties obtained through dynamic mass up-take measurements with a quartz microbalance. We use uniform films of homopolymers as model systems.

Accomplishments

We have concentrated on getting some of the missing information concerning the structure of polymers under confinement. One property for which very little is known is the distribution of orientation of the segments of chains with respect to the normal to the film surfaces.

In close collaboration with J.B. Kortright of the Materials Sciences Division, we have worked at developing the use of Resonance Soft X-ray Reflectivity (RSoXR) to provide the profile of orientational order of polymer segments in thin films. Using as model system homogeneous films of side-chain liquid crystalline polymer oriented parallel to the surfaces, we have demonstrated that resonant soft X-rays reflectivity measured with different polarizations at the carbon K-edge indeed show some linear dichroism at energies close to the resonance peak of aligned chemical groups (measurements performed the Advanced Light Source at LBNL and ELETTRA in Italy). We have also developed the necessary tools to calculate the expected reflectivity for a system with known orientational order. Using absorption spectra measured on the same films as input for calculating the material refractive indexes, we were also able to model the measured data at different energies by adjusting only a limited set of parameters including the order parameter in the system.

In parallel to these structural studies, we have performed absorption and diffusion measurements in thin films using a quartz microbalance in an environmental chamber. By depositing films on the resonator of a quartz resonator, putting it in the presence of a vapor or liquid of defined composition, and following the mass up-take in time through the resonance frequency of the quartz resonator, we are able to measure both the solubility and the diffusion of the molecules present in the chamber through the polymer film. Our first case study concerns the behavior of amorphous polylactic acid films in the presence of water vapor. We have shown that the amount of water absorbing in the films is significantly larger when the films are deposited on hydrophilic silica surfaces than when deposited on hydrophobic gold surfaces. The absorption dynamics is also totally different. By modeling the diffusion with a Fickian law, we can reproduce the absorption dynamics in films on gold by assuming that there is an interfacial layer with different diffusion properties. The absorption dynamics in films on silica surfaces cannot be modeled assuming a Fickian diffusion. 
Conducting Metal-Organic Frameworks

Principal Investigator: Jeffrey R. Long

\section{Project Description}

This project aims to induce electronic and ionic conductivity in porous metal-organic frameworks (MOFs). While MOFs have shown great potential for their applications in the adsorption of small molecules, few studies report electronic or ionic conductivity in such porous compounds. To induce electronic conductivity, we are developing new frameworks with select metal cations and ligands to enhance electron transfer throughout the framework. In one route, work is focused on frameworks based on octahedral $\mathrm{Fe}^{\mathrm{II} / \mathrm{III}}, \mathrm{Ru}^{\mathrm{II} / \mathrm{III}}, \mathrm{Os}^{\mathrm{II} / \mathrm{III}}$, and $\mathrm{Mo}^{0 / \mathrm{I}}$ redox centers, which have demonstrated electron delocalization in discrete complexes. We are attempting to link these cations into a porous framework using nitrogen and sulfur-based ligands to enhance metal-ligand bond overlap, in contrast to the use of carboxylic acid ligands observed in many reported frameworks. In addition to metal center-based redox, we are also examining the use of ligand-based redox centers to induce electronic conductivity in the corresponding extended framework.

We are also investigating the relationship between pore size/structure and lithium ion conductivity in a host of known MOFs. Neutral frameworks may be impregnated by lithium cations and small anions. Anionic frameworks containing exchangeable cations will be impregnated with lithium cations; and if any of the redox-active MOFs described above may be reduced to an anionic form, these may also be impregnated by lithium.

Accomplishments

We have successfully synthesized the metal-organic framework $\mathrm{Cu}\left[\mathrm{Ni}(\mathrm{pdt})_{2}\right]\left(\mathrm{pdt}^{2-}=\right.$ pyrazine-2,3-dithiolate), which is an isostructural analogue of the known framework $\mathrm{Cu}\left[\mathrm{Cu}(\mathrm{pdt})_{2}\right]$. We have conducted various electrical, electrochemical, and photoelectrochemical experiments to determine the framework's optical band gap, nature of conductivity ( $n$ - or $p$ type), and effect of doping on conductivity. Of particular interest is the fact that these two isostructural MOFs demonstrate that the band gap and conductivity of dithiolene-based MOFs can be modulated by changing the metal, and that with appropriate oxidative doping, the conductivity can be increased by a factor of $10^{4}\left(10^{-8} \mathrm{~S} / \mathrm{cm} \rightarrow 10^{-4} \mathrm{~S} / \mathrm{cm}\right)$ without losing porosity.

In terms of MOFs based on metal-centered redox activity, we have recently prepared some crystalline frameworks based on napthalene bispyrazolate and anthracene bispyrazolate coordinated with $\mathrm{Fe}^{2+/ 3+}$. Further work is underway characterizing their structure and porosity; we believe these materials will have interesting magnetic and redox properties. We also have demonstrated double-exchange in imidazolate-bridged octahedral vanadium complexes, and are further expanding this work to extended frameworks incorporating imidazolate and ruthenium.

The lithium ion conductivity of a number of known frameworks (MOF-177, etc) after impregnation has been screened. These impregnated frameworks have shown difficulty in forming pellets for conductivity measurements, and typically have low ionic conductivities with high energies of activation. In the future, we will further investigate redox in neutral frameworks in hope that after conversion to anionic form, they can be impregnated with lithium cations and exhibit high mobility. 
Solution-Processed Inorganic Composites with Mixed Transport Characteristics Principal Investigator: Delia Milliron

Project Description

This project aims to develop a generalized methodology for deposition of inorganic nanocomposites with controlled composition, morphology, and phase of the component materials. The focus is on demonstrating control over mixed transport properties by independent selection of the matrix material and the nanoparticle inclusions. The resulting complex functionality will enable optimized electrolyte switching, electrochromic, photovoltaic, and thermoelectric materials, for example.

Composites will be prepared from separately synthesized colloidal nanoparticles and molecular precursors, each processed individually from solution, then thermally converting the precursor to form the matrix material. To maintain the integrity of the nanocrystals, precursors will be synthesized which decompose at low temperature. The modularity of the proposed process will be leveraged to vary the composition of each component in order to independently tune transport characteristics such as ionic and electronic conductivity.

\section{Accomplishments}

A new, modular method for fabricating inorganic nanocomposites from colloidal nanoparticles and soluble chalcogenidometallate clusters (CMCs) has been demonstrated and the resulting composite films characterized by electron microscopy and x-ray scattering. Our fabrication process consists of first casting the nanoparticles from solvent dispersion to form superlattice thin films wherein the assembly is mediated by the native organic ligands. Next, these ligands are displaced by CMCs, which are finally decomposed at low temperature to form a continuous inorganic matrix phase in the intra-nanoparticle spaces. The regular ordering of the initial nanoparticle assembly, including preferred orientation in vertical nanorod arrays, is largely maintained through the process of converting to an all-inorganic composition.

Because the nanoparticles and CMCs are synthesized and processed independently, this process permits exceptional composition and morphological tunability: we have prepared over 20 different nanocomposites. In order to systematically examine mixed transport, synthetic methods have been developed for size-controlled 3-15 nm diameter colloidal particles of the silver chalcogenides $\left(\mathrm{Ag}_{2} \mathrm{X}, \mathrm{X}=\mathrm{S}, \mathrm{Se}\right.$, and $\left.\mathrm{Te}\right)$ and these have been combined with $\mathrm{CMC}$ precursors to $\mathrm{GeS}_{2}$ and $\mathrm{GeSe}_{2}$ to produce a class of composites which are mixed electronic and $\mathrm{Ag}^{+}$ionic conductors. Because the composites are thermally stable, the phase transition of $\mathrm{Ag}_{2} \mathrm{X}$ into the high temperature superionic phase, which occurs between $100-200^{\circ} \mathrm{C}$, can be observed by $\mathrm{x}$-ray diffraction without inducing nanocrystal sintering. We are now leveraging this observation to study the size-dependence of this phase transition in nanocomposites.

In order to study the mixed transport properties, we have begun to carry out impedance analysis on our composite thin films over a broad frequency range and as a function of temperature. Ionic and electronic transport give rise to distinct frequency-dependent features of the complex impedance spectrum, allowing their respective contributions to the overall conductivity to be evaluated. We intend to correlate the temperature dependence of each conductive channel with the structural phase transition information obtained by x-ray diffraction to gain a comprehensive understanding of the factors governing mixed transport in nanostructured composites. 
In situ Electromechanical Probing in a TEM

PI: Andrew M. Minor

Project Description:

The goal of this project is to establish the technical ability to probe both the electrical and mechanical properties of individual nanostructures simultaneously, quantitatively and at high resolution in a TEM. This capabiltity would enable the study of coupled electrical and mechanical phenomena for a wide variety of materials science applications, including energy scavenging, microstructural evolution in piezoresistive materials, phase transformations during mechanical deformation and exploratory work on individual nanostructures.

The research and development efforts will center around the design and initial implemenation of a custom-built holder for a transmission electron microscope. The holder will have the sensitivity and stability for in situ TEM electromechanical probing of individual nanostructures such as piezoresistive thin films or nanowires. To date, one of the main limitations on bending or compression experiments of individual nanowires below $\sim 100 \mathrm{~nm}$ in diameter have come from control of the testing apparatus and the noise floor for quantitative force data. The stability and control of quantitative mechanical testing has come a long way in the past few years, and new control electronics have just become available that will allow for improved displacement control and feedback in order to decrease the current amount of vibration in the indenter tip. Accurate displacement control is important for in situ small-scale mechanical testing where one would like to anticipate contact and deformation in terms of a spatial constraint (the field of view during the test).

Achievements:

The design of the in situ electromechanical TEM holder has been achieved and the holder is currently under construction (this is the first year of a two-year project).

During this past year we have concentrated on the sample preparation and materials synthesis necessary for studying coupled electrical and mechanical behavior in nanostructures. We have begun by picking a system that undergoes a pressure-induced phase transformation, orthorhombic $\mathrm{La}_{1-\mathrm{x}} \mathrm{Ba}_{\mathrm{x}} \mathrm{MnO}_{3}$ and deposited the synthesized nanoparticles on a microfabricated $\mathrm{Si}$ substrate. To investigate the phase transformation behavior we have performed in situ nanocompression tests inside a JEOL 3010 in situ TEM with a flat diamond platten. Initial results show that an intermediate phase is achieved under compression, and we have been able to directly correlate the phase transformation with the load vs. displacement data achieved during nanocompression by recording the diffraction pattern in situ. With the arrival of our new holder scheduled for the end of 2009 we anticipate being able to measure both the mechanical and electrical properties simultaneously. 


\title{
X-ray Studies of Charge-Order Dynamics in Complex Materials
}

\author{
Principal Investigator: Robert W. Schoenlein
}

\section{Project Description}

The purpose of this project is to develop novel ultrafast resonant x-ray scattering techniques, and apply them to study charge and orbital ordering dynamics in complex materials where strong interplay between charge, spin, and subtle lattice distortions give rise to rich phase diagrams. This project will focus on two classes of transition-metal oxides, and their dynamic response to tailored vibrational and electronic excitations: (i) CMR manganites exhibiting charge/orbital ordering, and (ii) blue-bronze (K0.3MoO3) compounds exhibiting charge-densitywaves. Resonant $\mathrm{x}$ ray scattering (RXS) is a powerful quantitative probe of charge and orbital ordering in complex materials. However, studies to date have focused on ordering phenomena in the 'quasi-static' regime, as a function of adiabatic changes in doping, pressure, temperature, and applied field. Understanding the dynamics of such ordering phenomena, their time scales, correlation lengths, and coupling to different degrees of freedom, is essential for revealing the driving force behind the intriguing physics and novel properties of correlated materials.

Resonant x-ray diffraction measurements are made on Bragg reflections that are nominally forbidden by the lattice unit-cell symmetry. Anisotropy of the electron density (e.g. from charge/orbital ordering) results in non-zero Bragg reflections. These reflections are particularly enhanced at absorption resonances (e.g. L-edge or K-edge) since they directly probe the valence states most affected by the symmetry-breaking. Time-resolved resonant x-ray diffraction experiments will be conducted at ALS BL6.0.1 and BL6.0.2, the only beamlines in the U.S. providing the required tunable femtosecond x-ray pulses in the $0.2-10 \mathrm{keV}$ range, accompanied by a laser system covering the visible and mid-IR range (for tailored excitation). For these studies, a dedicated vacuum chamber and diffractometer will be constructed that will incorporate a cryostat for low-temperature measurements.

\section{Accomplishments}

We have carried out static resonant soft x-ray scattering experiments at ALS BL 8 to characterize the orbital order of our samples, and have determined the critical experimental parameters (geometry, photon energy, scattering angle, and sample temperature) to observe the orbital-order peak. We found that the orbital order peak in PCMO shows an interesting temperature dependence which is quite different from published results obtained with hard x-ray scattering. Moreover, we observed a decrease of the orbital order peak at low temperature which might be related to the melting of orbital order by x-rays. The study of the temperature and x-ray induced melting of orbital order provides essential information for time-resolved studies at BL 6 .

For soft x-ray studies at BL 6.0.2, we have modified a UHV diffractometer chamber (include re-design of the front flange, adding a new detector, and replacing the support stand) and used this chamber for the first BL6 experiments which revealed the static orbital-order peak. We also did some testing in time-resolved mode and we have identified the appropriate detector size and aluminum foil for laser filter needed for our time-resolved measurements.

Preparations have been made for hard x-ray experiments at BL 6.0.1 to study the melting of the charge order in PCMO. We have developed a theta/2-theta rotation capability in the BL 6.0.1 endstation chamber and have used this to characterize the charge order peak at $100 \mathrm{~K}$.

We are presently working on the first time-resolve experiments of charge and orbital ordering dynamics in manganites. 
Quantifying the Quantum Backaction of a Non-Linear Dispersive Measurement Principal Investigator: Irfan Siddiqi

\section{Project Description}

A canonical method to read out a quantum spin system is to couple it to an oscillator. This technique, first developed in atomic physics, is known as a dispersive measurement. When the quantum spin interacts with the oscillator, its effect is to modify the oscillator frequency. Thus the oscillator has two different resonant frequencies corresponding to the up and down states of the spin. One can determine the frequency of the readout oscillator by energizing it with a microwave frequency electromagnetic pulse and observing the phase shift in the resulting reflected or transmitted signal.

In quantum mechanics, any act of measurement, even this seemingly benign dispersive one, perturbs the system being measured. In this case, the energy splitting of the spin systemthe Larmor frequency - becomes shifted when the oscillator is energized. This is known as the AC Stark effect. If the oscillator is energized with a larger number of photons $\mathrm{N}$ in the measurement pulse, the Stark shift is enhanced and the dephasing becomes greater. At the same time, the measurement has a greater signal to noise ratio with a larger photon field and the state can be determined in a shorter time. One can show that there is an uncertainty relation between the measurement time and the dephasing rate, which is simply a restatement of Heisenberg's uncertainly principle. One natural question that comes to mind is whether a nonlinear oscillator which can act as an amplifier can extract more information.

\section{Major Accomplishments}

We have developed/adapted/tested three new cryogenic microwave elements. First, we have successfully adapted two types of microwave switches that work at temperatures down to $10 \mathrm{mK}$. One switch is a mechanical transfer switch which allows us to insert a quantum noise limited superconducting amplifier into the microwave measurement chain in-situ. The other is an ac diode switch based on GaAs to chop between two cryogenic loads. Both of these elements are essential for a precise calibration of our measurement system gain and noise temperature-a necessary step for quantifying the number of photons $\mathrm{N}$ being used in our quantum measurement of the spin. We have used these switches to characterize the noise emission of our custom designed filter elements which are loaded with microwave absorptive material and are used to suppress external electromagnetic radiation which can cause decoherence in the spin circuit. Our results, the first measurements at low temperatures of these types of absorbers, demonstrate that such filters retain their dissipative character down to $\mathrm{mK}$ temperatures and are in equilibrium with their cryogenic environment.

With this hardware, we have successfully tested superconducting qubits based on a hybrid charge phase design. We have measured the coherence time of these qubits and they are currently in the 10 ns range. These experiments were conducted with the measurement circuit tuned for high gain since the coherence time is significantly lower than the expected value of 1 $\mu \mathrm{s}$. As such, we do not have enough signal to noise to vary the measurement strength to the few photon or weak measurement regime. We are now in the process of using a different qubit architecture which has reliably produced long coherence times to complete this measurement. 


\author{
Self-Assembly of Membrane Proteins \\ Principal Investigator(s): Berend Smit and Ting Xu
}

\title{
Project Description
}

Proteins or peptides embedded in membranes have an interesting collective behavior. For example, clustering of proteins is seen as an important factor in membrane-protein-related diseases like Alzheimer's and Creutzfeldt-Jakob. Some organisms use anti-microbial peptides as a defense against bacteria, which cluster in the cell membrane of a bacteria resulting in cell lysis. Finally, membrane proteins can be used as building blocks for novel materials. In the mosaic model of membranes embedded proteins or peptides are seen as isolated molecules in a fluid. Recently, it has become clear that these molecules organize in clusters and that this may affect function and signaling. Yet the nature and structure of these clusters is as of yet poorly understood.

The purpose of this project is to obtain a better understanding of the collective behavior of trans-membrane proteins, as mediated by the lipid bilayer itself. With this aim we will further develop a mesoscopic simulation model, using dissipative particle dynamics. This model will allow us to systematically investigate, using molecular simulations, how these membranemediated protein-protein interactions depend on the molecular structure of the lipid bilayer constituents.

\section{Accomplishments}

Our most significant achievement is the development of a cholesterol model that captures experimental bilayer phases, the condensation effect, and other properties when in a binary mixture with a saturated lipid. This development is crucial for studying protein aggregation in a biophysically-relevant lipid bilayer because cholesterol is a substantial component of most cell membranes. In particular, the robustness of our coarse-grained model in capturing different phases and structures as temperature and composition vary is quite promising. To our knowledge, this is the first molecular model to represent phases and other bilayer properties with reasonable fidelity. Since membrane-mediated protein aggregation can be influenced by proximity to these transitions, we view this as a critical achievement. Preliminary simulations show that this model cholesterol induces and is incorporated into protein clusters of cylindrical trans-membrane peptides, whereas such clustering is not as strongly evident in the corresponding pure lipid bilayer.

We have also developed a new biased Monte Carlo algorithm specifically for the insertion of solutes in the lipid bilayer. This technique, biasing insertions towards regions with greater density of solute, allows us to determine the equilibrium partitioning of small molecules like alcohol between the aqueous phase and the lipid bilayer in a computationally efficient manner. Alcohol is often used as a simpler substitute for anesthetic molecules in computational studies since it is known to perturb bilayer protein functioning in a manner similar to anesthetics. Therefore, this technique will be quite useful for studying possible membrane-mediated biophysical effects of anesthetics on protein clustering and function, which are hypothesized to supplement various specific biochemical modes of action.

We are in the process of further characterizing protein clustering and protein-protein potentials of mean forces in the presence of cholesterol and alcohol in order to gain further insight into membrane-mediated protein aggregation phenomena. 


\section{Understanding How Nanoscale Interfaces Modify Predicted Optical, Vibrational, and Electronic Properties}

Jeff Urban, Adam Schwartzberg, Delia Milliron, Jeff Neaton, David Prendergast, and Shenyuan Yang

Purpose: Predicting and measuring the electronic energy levels, gaps, and alignments in nanoscale systems as done for bulk materials, is currently impossible. Moreover, remarkable modifications to bulk optical, vibrational, and structural properties have been reported, however, predictive models and a true physical understanding of these phenomena remain elusive. We are currently investigating novel nanomaterials that address major scientific questions such as: What physics controls electronic level alignment at nanoscale interfaces? How are vibrational states modified by interface termination? Do the wavelength-dependent refractive index numbers still remain valid for subwavelength structures?

\section{Core Results: Effects of Nanoscale Interfaces on Dielectric and Optical Properties}

Our most significant result is the discovery that nanoscale wires of GaN have greatly enhanced nonlinear upconversion of sub-bandgap photons, and, that these properties may be used to efficiently generate white light from a single $\mathrm{GaN}$ wire excited at low-power, without the use of phosphors, heating, or expensive illumination sources.

Our initial studies on GaN nanowires have uncovered novel phenomena that derive directly from the crystal interfaces of the wire. By illuminating a wire appropriate wavelength laser light and collecting the light emitted from the wire, we observe two unexpected results: 1.) incredible structure in the signal from the ends of the wire and 2.) photoluminescence from the wire associated with bandgap excitation. The first observation (structure in the radiation emitted only at the wire tips) can be understood as the observation of propagating Fabry-Perot etalon modes in the wire. This is only possible for highly reflective interfaces (such as the facets of the wire), and may be modeled using the Fabry-Perot formula. However, another interesting result emerges from this - measured changes in the refractive index of different measured wires (as determined by the Fabry-Perot formula) of nominally identical chemical and structural properties. We believe that this result may be understood due to the extraordinarily large interface on the wires which lead to enhanced second order optical coefficients and generation light in the wire, generating a large density of carriers, thus modifying the refractive index of the material. We see excitation of the bandgap in the photoluminescence signal in addition to the broad, so-called "yellow band" underlying the Fabry-Perot fringes. This total emission produces a continuous, "white light" spectral breadth never before seen. This, along with the nonlinear dependence of emission on excitation power, shows that the material interfaces are leading directly to enhancement of nonlinear optical properties, changes in the measured refractive indices away from bulk values, and unusual efficiency in the wave-guiding of light in these small dielectric light pipes. We are currently in the process of developing new "on-chip" spectroscopy applications for these nanoscale white-light sources and exploring the detailed physics leading to these unexpected dielectric and optical anomalies. 


\section{Microscopic Mechanism of Resistance Switching Memory Effect \\ Principal Investigator(s): Junqiao Wu}

\section{Project Description}

We proposed to directly probe the phase transition in transition metal oxide (TMO) nanowires that leads to the resistance switching utilizing a number of in situ tools for a full understanding of its microscopic mechanisms. This approach takes advantages of the reduced size, unique one-dimensional geometry, and superior mechanical flexibility of the nanowires, where any local phase transition will be naturally exposed to beam analysis in a variety of microscopic tools. The objectives of this project are to 1) develop a strategy to synthesize selected TMO nanostructures with superior resistance switching properties, and 2) explore the phase transition in these nanoscale TMOs for a fully understanding of the microscopic mechanisms. In the one year funding period, we focused on a representative $\mathrm{TMO}, \mathrm{VO}_{2}$, where a sharp metal-insulator phase transition occurs at $68^{\circ} \mathrm{C}$.

\section{Accomplishments}

Our most significant accomplishments are discoveries of: i) a strain stabilized and engineered metal-insulator domain structure along $\mathrm{VO}_{2}$ nanobeams (Nature Nanotechnology, 4, 732(2009)). This discovery sheds bright light on the famous phase inhomogeneity problem in correlated electron materials in general; ii) an abnormal thermoelectric effect across the domain walls in $\mathrm{VO}_{2}$ microbeams (Nano Lett., in press (2009)), which is important to implementing the widely discussed junction-enhanced thermoelectrics; iii) superelasticity in the metal-insulator phase transition (Phys. Rev. B Rapid Commun, in press (2009)); iv) a universal carrier concentration of the insulating phase of $\mathrm{VO}_{2}$ before it transits into the metallic phase (in review at Phys. Rev. Lett.). It has been a debate for many decades in condensed matter physics whether the phase transition in $\mathrm{VO}_{2}$ is a Mott transition or a Peierls transition. This discovery proves the Mott nature of the phase transition; and v) an extended phase diagram covering all phases of $\mathrm{VO}_{2}$ over a phase space that is ten times broader than previous attained (to be submitted to Nature Mater.). This phase mapping was enabled in our case by the superior mechanical flexibility of the nanobeams which supports exceedingly high values of strain compared to the bulk.

We are currently extending the developed techniques and strategies to various TMO nanostructures that exhibit a variety of phase transitions, hoping to better understand their physics and dynamics at the nanoscale. The Director's Review Committee commented on our work, “... This demonstration is nice piece of work by a highly capable researcher which may very well form the basis for widespread applications in future, as in the semiconductor industry...”

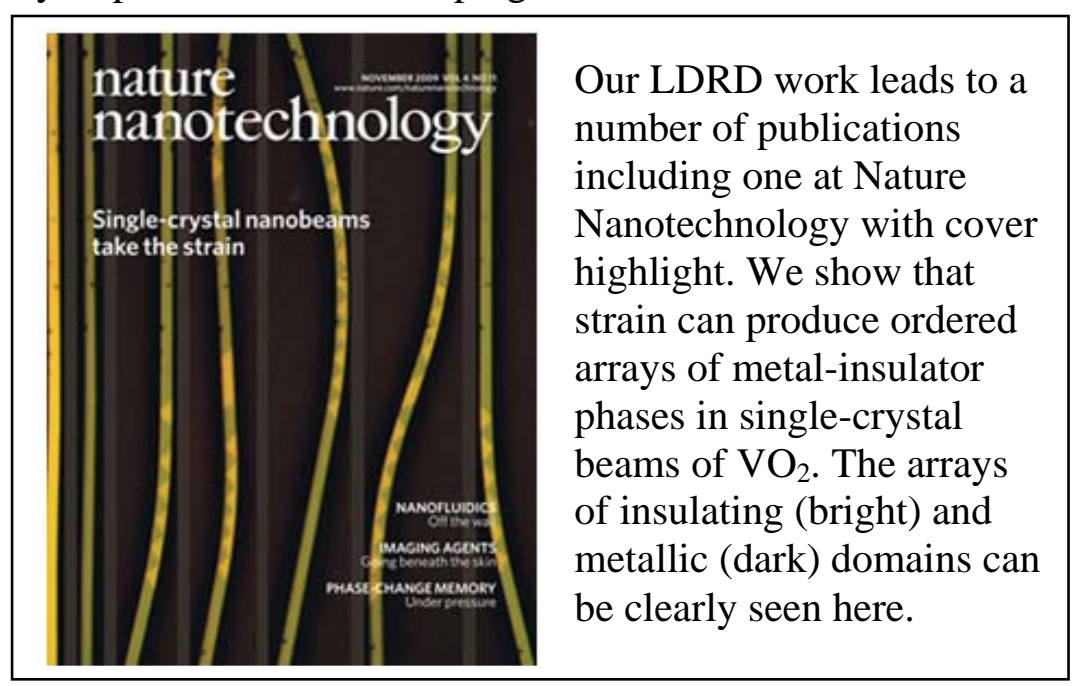


New Experimental Initiative to Deduce $(n, f)$ Cross Sections for Advanced Fuel Cycle Studies

Investigators: M. Shamsuzzoha Basunia, Richard B. Firestone and Rod M. Clark

\section{Project Description:}

This is the third and final year of a new research effort to determine basic nuclear physics data for Advanced Fuel Cycle (AFC) studies. Sustainable and safe nuclear energy has been identified as an important national need and the related basic research is part of the DOE mission. We are attempting to determine cross-sections for neutron-induced fission of actinides involved in fast reactor fuel cycles. Existing data often show significant discrepancies and do not extend to the higher neutron-energy range (up to $\sim 20 \mathrm{MeV}$ ) found in fast reactors. The goal is to develop novel surrogate reactions to deduce the relevant cross sections to unprecedented accuracy $(<10 \%)$ over a broad neutron energy range $(0.2$ to $20 \mathrm{MeV})$. The surrogate reaction method uses a light-ion reaction to determine the decay probabilities of a compound nucleus of interest. The formation probability can be modeled accurately. By combining the calculated formation probability with the measured fission probability the desired $(\mathrm{n}, \mathrm{f})$ cross-section can be determined. We use light-ion beams, accelerated by the 88-Inch Cyclotron, which are incident on actinide and other targets. Charged particles and fission fragments are detected in the STARS Si-detector array in order to determine the required fission probabilities.

Accomplishments:

Over the last year, the LBNL/LLNL collaboration has studied various aspects of surrogate reactions. Researchers from UC Berkeley and the University of Richmond are also participated in the collaboration. Experiments using deuteron beams are explored to populate and study the properties of continuum region of nuclides through $(\mathrm{d}, \mathrm{p})$ transfer reaction at the 88-Inch Cyclotron using the STARS + LiBerACE array. The ${ }^{92,93,94} \mathrm{Mo}(\mathrm{d}, \mathrm{p})$ reactions were performed to measure the lifetime of quasi-continuum levels. Analysis of data is in progress. The results along with future studies on properties of continuum region to feed the discrete levels at lower energy levels of nuclides would eventually help to improve the statistical Monte Carlo codes for $(\mathrm{n}, \mathrm{g})$ cross section calculations more effectively.

In addition, in this year, LBNL scientists have completed analysis of experimental data of the ${ }^{237} \mathrm{~Np}(\mathrm{n}, \mathrm{f})$ reaction. The cross sections were determination using the ${ }^{238} \mathrm{U}\left({ }^{3} \mathrm{He}, \mathrm{tf}\right)$ surrogate reaction, manuscript was prepared and published. Another manuscript to benchmarking the results of $\left(\mathrm{p}, \mathrm{p}^{\prime}\right)$ surrogate from the ${ }^{155,156} \mathrm{Gd}(\mathrm{n}, \mathrm{g})$ reaction studies is prepared for submission to the Physical Review C. Several more publications are expected soon. We also helped to relocate the STAR and LiBerACE facilities from Cave 4C to Cave 2, with upgrading features, at the 88-Inch Cyclotron.

Overall, this 3-year LDRD was very fruitful to study the surrogate reaction approach for nuclear cross section determination at the 88-Inch Cyclotron facility, LBNL, for future work. Recently a 5 -year stimulus funding to the nuclear data group, Nuclear Science Division, LBNL, is obtained from the DOE. 


\section{A $100 \mathrm{~km}^{3}$ neutrino detector for ultra high energy neutrinos Principle Investigator: Spencer Klein}

\section{Project Description}

The purpose of this project is to develop a prototype detector that could be used as an element in a large array which would comprise a $100 \mathrm{~km}^{3}$ detector for ultra-high energy (above about $10^{17} \mathrm{eV}$ ) neutrinos. GZK neutrinos are one source of these neutrinos; they are produced when protons with energies above about $4 * 10^{19} \mathrm{eV}$ interact with photons from the $3^{0} \mathrm{~K}$ cosmic microwave background radiation, forming a $\Delta$ resonance. The $\Delta$ decay leads to a lower energy proton and, often, a neutrino. Repeated GZK interactions limit the range of higher energy (above $4 * 10^{19} \mathrm{eV}$ ) cosmic rays, so cosmic-ray detectors only probe nearby (within $75 \mathrm{Mpc}$ ) galaxies. In contrast, GZK neutrinos can travel cosmological distances, and probe the high energy universe out to redshifts of $\sim 2-4$. However, the small flux requires a very large detector.

Existing optical Cherenkov techniques cannot scale to a $100 \mathrm{~km}^{3}$ detector. Radio and acoustic detection of neutrino induced showers have been proposed as alternatives. Acoustic detection suffers from short acoustic attenuation lengths, so we are focusing on radio detection. Radio detectors observe the coherent radio ( $50 \mathrm{MHz}$ to $1 \mathrm{GHz}$ ) pulse emitted by neutrinoinduced particle showers. The Ross Ice Shelf is an attractive place for a radio-neutrino detector. There, $650 \mathrm{~m}$ of ice sit atop the Artic Ocean. The water-ice interface acts as a high-quality radiowave reflector. Downward-going neutrinos that interact in the ice produce downward-going Cherenkov radiation, which is reflected to the surface. In contrast to radio-detection elsewhere (e.g. at the South Pole), it is not necessary to dig deep holes to bury the detectors. The ARIANNA group would like to build a 10,000 station, $100 \mathrm{~km}^{3}$ neutrino detector; we are focusing on prototyping efforts toward this detector.

\section{Accomplishments}

We are working in two main areas: studies of electromagnetic cross-sections and their affect on shower development, and development of prototype electronics hardware for the detector.

These cross-sections are an important input to ARIANNA simulations, since the radio frequency spectrum and angular distributions depend on the charge distribution in the shower. We have calculated improved cross-sections for bremsstrahlung and pair production in the regime where the Landau-Pomeranchuk-Migdal (LPM) effect is important. Our calculations include other phenomena, such as photonuclear interactions, and the suppression of bremsstrahlung due to pair production (and vice versa) which become important when the LPM effect reduces the cross-sections significantly.

We have developed a set of requirements for the electronics and are working on several different areas of prototype electronics development. We have designed and built a new lownoise amplifier chain which only consumes $250 \mathrm{~mW}$. We tested a commercial GPS receiver which provides the required 15-ns time resolution, while consuming only $155 \mathrm{~mW}$, and is rated to low temperatures. We have refurbished and debugged the original ARIANNA/ANITA prototype (ANITA electronics in an ARIANNA packaging), installing a processor and memory boards rated for low temperatures, and upgrading the FPGA code. Thorsten Stezelberger and Klein will deploy this hardware to the Ross Ice Shelf this Austral summer. 


\section{Physics Detector and Sensor Technologies Applied to Geological and Geophysical Applications at DUSEL \\ Principal Investigator: Kevin T. Lesko}

Project Description:

The purpose of this project was to develop solutions to R\&D issues enabling new opportunities provided by the Deep Underground Scientific and Engineering Laboratory (DUSEL). Partnerships between Earth Scientists, Engineers and Physicists were developed to investigate underground carbon remediation techniques, and develop novel detector components and materials for underground research. The first activity researches underground carbon sequestration, developing techniques for transporting, and monitoring $\mathrm{CO}_{2}$ and understanding long term sequestration by characterizing the site and developing monitoring techniques. The second topic investigated detector materials with a focus on ultra-low contamination of radioactive species and novel detector designs to mitigate the impact of contamination.

The primary objective of the $\mathrm{CO}_{2}$ study was to assess the feasibility of establishing a facility at the Homestake DUSEL laboratory to conduct large-scale flow experiments related to geological carbon sequestration (GCS). A challenge in implementing GCS is ensuring long-term entrapment of the injected $\mathrm{CO}_{2}$, minimizing environmental impacts, and quantifying the risk of $\mathrm{CO}_{2}$ leakage. The proposed DUSEL $\mathrm{CO}_{2}$ experimental facility, consisting of a series of vertical pressure vessels filled with sand, clay, and cement, could utilize the large vertical extent $(>400 \mathrm{~m}$ ) of existing borings at Homestake. An initial suite of experiments has been envisioned to study the behavior of vertically migrating $\mathrm{CO}_{2}$, its potential interaction with reservoir and cap rocks and well cement, and the effects of microbes on $\mathrm{CO}_{2}$. This work consisted of a review of existing geologic, mine, and environmental data from the Homestake mine, a visit to the mine to evaluate potential sites for installing long pressure vessels underground, and the deployment of environmental sensors within different facility levels.

We drew on physicists and engineers in the Germanium Detector Lab to develop assay techniques, ultra-clean materials, and novel detector designs appropriate for next generation neutrinoless double beta decay and dark matter experiments.

Accomplishments:

Carbon Sequestration: new experimental deployments in Homestake were developed making use of existing access to developed levels in the facility. These locations will enable the development of multiple experimental test-beds in a $\sim 2 \mathrm{~m}$ diameter shaft. The new shaft is proposed to extend $\sim 500 \mathrm{~m}$. We developed procedures and techniques to characterize the sequestration test bed and to monitor the evolution of injected $\mathrm{CO}_{2}$, including phase transition, migration and dispersion. We assessed the current national $\mathrm{CO}_{2}$ sequestration demonstration projects and sequestration repositories. From these projects we have identified on key research foci for the instrumentation including determining leakage, and migration within underground repositories. Assessing well-caps and borehole casing need detailed monitoring. The insertion of supercritical $\mathrm{CO}_{2}$ is pursued as the preferred form for introducing carbon in the underground. We developed plans to assemble large-scale research project in DUSEL to observe and document the complex interactions of supercritical $\mathrm{CO}_{2}$ with the repository media and containment infrastructure. Initial environmental monitoring was begun with temperature, pressure, and relative humidity measurements routinely acquired at the Homestake site. These plans resulted in the establishment of a collaborative effort with Princeton and submission of a proposal responding to the NSF solicitation.

New Materials and Sensors: Next generation detectors require special materials including ultra-low background materials, ultra compact and ultra-pure electronics for detectors, and remote sensor arrays. We developed novel, low-mass, front-end amplifiers using a thin-film resistive feed-back design. These designs achieved record low noise performance when coupled to prototype detectors. These front-end electronics are critical to achieve anticipated performance requirements for physics experiments requiring low capacitance, high bandwidth solid-state detector designs. We completed characterizing the novel low capacitance germanium detectors previously reported. We pursued detector segmentation designs to further increase the detector's ability to reject internal and external backgrounds. The front-end electronics has been accepted as the baseline design for Majorana Demonstrator project. 
Ultra-Sensitive Ge Detectors for Low-Background Physics Experiments

Principal Investigator(s): Kai Vetter

\section{Project Description}

The purpose of this project is to significantly increase the sensitivity in the detection of rare physics processes such as dark matter interactions, neutrino-less double-beta decay, or coherent neutrino scattering. The detection of such processes requires ultra-low background and ultra-low electronics noise. The recent development in fabricating and operating low-capacitance p-type point contact (PPC) Ge detectors provides the potential to realize unprecedented noise levels in large, kg-size Ge detectors. This project aims at the development of a electronics readout to realize ultra-low noise implemented with very low mass and low-radioactivity components required for the detection of rare processes. In addition, low-energy threshold and ultra-high resolution detectors of $1 \mathrm{~kg}$-scale can have a profound impact in atomic physics experiments as well as in nuclear forensics and safeguards applications. The purpose of this project is to explore the full potential of these detectors as well as the readout electronics in order to optimize detector performance for a wide range of applications.

\section{Accomplishments}

During this first year we were able to develop and demonstrate a very compact and low-mass detector readout with an electronic noise of less than $100 \mathrm{eV}$ in combination with a small PPC detector. The low-mass front end (LMFE) board is based on the resistivity feedback architecture, which is simpler than pulse reset designs, avoids interference from the reset signals, and requires less cables reducing the risk of contamination. A thin film of amorphous material to form the feedback resistor is being used. The amorphous films have high resistivity so that a high value resistor necessary to achieve low noise can be fabricated in a very small size, thus minimizing mass and with low radioactivity. Finally, a substrate material was chosen to fabricate the LMFE that satisfies several requirements: low dielectric loss for low noise, appropriate thermal insulation to allow the input FET to operate at the optimal temperature without additional heating, and potentially low radioactivity. We were able to refine the design and fabricate the readout board to optimize the operational temperature of the FET and the thin-film resistor as well as the operational conditions for the sputter process to create the thin film. We have identified, obtained, and implemented a low-noise and low-mass FET, which matches the small capacitance of the detector contact. The latest results indicate that with this LMFE configuration, an electronic noise of better than $60 \mathrm{eV}$ can be achieved. These noise values are unprecedented for charge-sensitive preamplifiers in a resistive feedback configuration.

In addition, we have evaluated a variety of cable designs and implementations through simulations and experiments. Cables serve as interface between the LMFE board and the preamplifier. Cables and associated connectors are important to maintain the signal fidelity and at the same time minimize the potential contamination due its radioactivity.

The goal for next year is to evaluate options to further reduce the electronics noise in combination with a PPC detector with the goal of $30 \mathrm{eV}$, which enables an energy threshold of about $100 \mathrm{eV}$ with a potentially profound impact in a physics program that could then be established. 
Theoretical Study of Nucleon Structure

Principal Investigators: Feng Yuan, Bowen Xiao

\section{Project Description}

The Understanding the structure of the Nucleon is a fundamental question in sub-atomic physics, and it has been under intensive investigation for the last several years. Modern research focuses in particular on the spin and the gluonic structure of the nucleon. They are key questions in current hadronic physics and also major driving forces for the RHIC and JLab programs. We propose an innovative research effort for theoretical study in this exciting area of strong interaction physics and focus on two topics: 1) the transverse spin physics and quark transversity distribution with the goal to extract the quark orbital angular momentum contribution to the proton spin; 2) the gluon structure at low Bjorken $\mathrm{x}$ and to study gluon saturation phenomena by carefully examining the gauge invariance and factorization properties in high energy processes.

Accomplishments

As an important step toward the comprehensive understanding of the novel single spin asymmetry phenomena in hadronic interaction process based on quantum chromodynamics (QCD) theory, we have finished a calculation of the next-to-leading order QCD corrections for a particular observable, the transverse momentum weighted single spin asymmetry in the DrellYan lepton pair production. This result has stimulated a number of further studies on the evolution equations for the associated correlation functions.

Applying the universality argument, we identified the twist-three function corresponding to the Collins mechanism in the fragmentation contribution. Calculating the large transverse momentum behavior of the Collins function from the twist-three fragmentation function, we find that it is universal. The same Collins function applies to the SIDIS process, the hadron distribution inside a jet in pp collisions and di-hadron correlation in e+e- annihilation, which is consistent with previous results.

We studied semi-inclusive hadron production in deep inelastic scattering at very small-x, where gluon saturation is relevant. Applying power counting, we found the transverse momentum dependent factorization to be consistent with the color-glass-condensate prediction for this process. We further calculated the transverse momentum dependent quark distribution at small-x from the saturated gluon source in the nucleon/nucleus and found its transverse momentum dependence to be very sensitive to the saturation scale. 


\section{Physical Biosciences Division}

The development of reusable software modules for the analyses of bioSAXS data Principal Investigators: Paul D. Adams, Peter H. Zwart, and Alex Hexemer.

Project Description

Important questions about certain biological processes often involve studying interactions and dynamics of protein-protein or protein DNA/RNA complexes. It is not uncommon that the structures of the individual components have been determined already but the structure of the complex is unknown or that the structure is significantly different in solution. In these cases, small angle X-ray scattering (SAXS) is a versatile tool that allows the determination of solution structures. It is also a powerful tool to study the dynamics of proteins: large conformational changes or domain movements are often associated with important biological processes.

Exactly because of these capabilities, the researchers in the field of structural biology are increasingly more interested in using SAXS as a standard technique to answer complex biological questions. This increase of interest in 'bioSAXS' applications places a demand on the availability of robust, user-friendly methods with a level of automation and self-guidance such that non-experts can produce meaningful conclusions without running the risk of greatly overinterpreting their data. Currently, the existing mainstream software for bioSAXS is not geared towards automation and transparency of the quality of the results. Furthermore, new developments in the analyses and prediction of protein structure and flexibility cannot be readily integrated with current SAXS software.

The goal of this proposal is to develop an expert software system for the analyses of bioSAXS data from this and other SAXS beamlines worldwide. New tools are vital because current software is difficult to use and prone to user misinterpretation of the data. We will address these problems and make bioSAXS a technique that can be accessed by a broad community of users. The new software will allow analyses to be carried out in a highly automated manner such that novice users can easily and reliably obtain results.

Accomplishments

In the first year of LDRD funding we have developed foundational algorithms for the data reduction and model-free interpretation of scattering curves and the subsequent refinement of models against this data. A data reduction program has been written that allows users to process their data in a highly automated fashion. This command line driven program, allows researchers to efficiently reduce their data without significant manual intervention. The algorithm produces radial integrated data for each specimen and, if possible, a background subtracted data set.

Summary statistics from reduced data provide valuable clues on the overall size and shape of the molecule under consideration. One of the most important summary statistics describing the data is the radius of gyration $\mathrm{R}_{\mathrm{g}}$. The radius of gyration is related to the mean inter atomic distance in the molecule under investigation, and thus provides vital information about the approximate size of the molecule in solution. A reliable determination of $\mathrm{R}_{\mathrm{g}}$ is made difficult due to the possible presence of aggregation, experimental noise and outliers. A robust, novel procedure has been developed that permits reliable estimates of the radius of gyration, under a wide range of data set qualities.

In order to reduce the discrepancy between a known model and SAXS data, an optimization algorithm is used to drive the molecule towards a confirmation that best agrees with the experimental data. This requires the rapid computation of scattering data from the current model. This is achieved efficiently using a spherical harmonics method that we have implemented.

We are in the process of developing other algorithms building on the foundation described above and will soon begin user testing of the software. 


\title{
Structural and Functional Characterization of DNA Translocation Across Membrane by SpoIIIE Using Advanced Single Molecules Microscopies
}

\author{
Principal Investigator: Carlos Bustamante
}

\begin{abstract}
Project Description
Within the cellular environment, DNA movement and organization are essential to the process of choromosomal replication, repair and segregation. The process of moving DNA requires mechanical work, which is generated by proteins that behave as molecular motors. Our long-term goal is to understand the dynamics of DNA translocases by studying the model system of sporulation in Bacillus subtilis and the molecular motor involved, SpoIIIE. First, we propose to characterize the mechanochemical cycle of the motor by using optical tweezers. This analysis will allow us to establish the coupling between SpoIIIE translocation and ATP reaction cycle, which consist of binding, hydrolysis and product release of ATP. Second, we also wish to understand how the molecular machine involved in this translocation reaches the right localization and topology at the molecular scale inside the cell and pumps the DNA in the correct direction. Toward these goals, we propose to use novel single-molecule microscopies, which should provide us unprecedented clues to understand the dynamic behavior of this family of DNA translocases and the design principles that optimize their operation.
\end{abstract}

\section{Accomplishment}

We designed and tested an optical tweezers experimental set-up that allowed us to investigate the effect of force on the translocation velocity of SpoIIIE motor along the DNA. Our results so far have shown that SpoIIIE is active under this new experimental set-up and is capable of translocating DNA against force up to $60-65 \mathrm{pN}$. The presence of numerous force spikes in these traces suggests that the protein, a ring ATPase, tends to slip backwards on its DNA substrate when it encounters a high opposing force. These results demonstrate that the protein is a very powerful and robust motor. Moreover, the velocity of translocation of SpoIIIE is force-dependent under saturating conditions of ATP. This suggests that the opposing force inhibits the force-generating step, such that it is the rate step of the reaction. Given the saturating conditions of ATP, the binding of ATP should not be the rate-limiting step of the mechanochemical cycle of the motor and therefore it suggests either the hydrolysis or product release of ATP is the force-generating step of this motor.

In order to investigate the SpoIIIE complex formation and the directionality of chromosome translocation during sporulation, we successfully constructed a photo-activated localization microscope (PALM), a recently developed high-resolution imaging technique for biological systems with a spatial resolution of $\sim 20 \mathrm{~nm}$. This instrument has enabled us to visualize the SpoIIIE complex in the sporulating $B$. subtilis in vivo. Preliminary PALM images of SpoIIIE fused to a photoswitchable fluorescent protein, tdEos, allowed us to observe various distributions of many SpoIIIE molecules along the division septum, in contrast to a single blurred spot seen in previous microcopy studies. These results indicate that PALM images of SpoIIIE-tdEos have the potential for unveiling high-resolution details of the organization of the SpoIIIE complex inside of the cell. 


\section{Biological Methods for the Synthesis of Iron-Based Nanomaterials Principal Investigator: Michelle Chang}

\section{Project Description:}

The purpose of this project is develop a deeper understanding of the chemistry that underlies the synthesis of iron-based nanocrystals by magnetotactic bacteria with the longterm goal of building biomimetic approaches to templating new spinel-type nanomaterials. Iron biomineralization is a complex yet insufficiently understood process that is key to understanding many important biological functions such as iron storage. Currently, there is very little molecular level information on biological magnetite synthesis. Furthermore, unlike more well-studied biomaterials based on calcium and silica, iron-based materials require redox chemistry during biogenesis. We thus seek to begin elucidating the mechanism of iron templating by magnetosome-associated proteins, as well as the chemical reaction mechanism of iron mineralization starting from monomeric iron. We are currently focused on studying the Mms family of proteins, thought to be directly involved with iron templating. These proteins contain two domains, consisting of an N-terminal silk-like motif thought to be involved in self-assembly of monomers as well as a C-terminal acidic domain that is proposed to bind and template metal ions. We will utilize a combination of biochemical and biophysical methods to study the mechanism of self-assembly and metal ion organization.

\section{Accomplishments:}

In the past year, we have developed a reproducible protocol for the preparation and purification of Mms6, which is thought to be one of the key players in the iron templating process. After working through several different approaches, we have found that we can obtain pure protein at high yield by production as a fusion protein in Escherichia coli, followed by proteolysis and isolation by two additional steps of chromatography. We have also been able to produce different mutants and derivatives by this approach that will be then characterized for their binding to iron as well as their ability to self-assemble.

At this time, we can scale up the preparation and are now ready to start biophysical experiments as well as experiments in characterizing materials templated by these heterologously-expressed proteins. We have been able to monitor concentration-dependent changes in protein structure and monomer assembly by both circular dichroism spectroscopy and dynamic light scattering. 


\section{LB08005}

Understanding the Nano thermodynamics of Molecular Machines Principle Investigators(s): Gavin E. Crooks

\section{Project Description}

The goal is to develop the necessary fundamental understanding of nanoscale, non-equilibrium thermodynamics so that we may understand the practical limits and operational principle of molecular machines, both biological and artificial. We are interested in discovering design principles that will allow us to engineer and customize our own efficient and effective molecular gadgetry. We wish to understand the thermodynamic efficiency of molecular machines, the tradeoff of efficiency with power output, how to determine the optimal thermodynamic processes, and how to engineer machines that can approach these optimal performances.

\section{Accomplishments}

We continue to develop the concept of thermodynamic length as a means of understanding the thermodynamics of molecular machines. Thermodynamic length is a path function that generalizes the notion of length to the surface of thermodynamic states. We have shown how to measure thermodynamic length in far-from-equilibrium experiments using the work fluctuation relations. For these microscopic systems, it proves necessary to define the thermodynamic length in terms of the Fisher information. Consequently, the thermodynamic length can be directly related to the magnitude of fluctuations about equilibrium. The work fluctuation relations link the work and the free-energy change during an external perturbation on a system. We use this result to determine equilibrium averages at intermediate points of the protocol in which the system is out of equilibrium. This allows us to extend Bennett's method to determine the potential of the mean force, as well as the thermodynamic length, in single-molecule experiments.

In collaboration with the Liphardt group, we have studied the self-organization of the escherichia Coli chemotaxis network. The assemble of transmembrane receptors into large clusters on the cell surface is a non-equilibrium process, which provides insight into self-assembly at the nano scale.

Most recently, we have developed a method for measuring the free energy of thermodynamic system away form equilibrium. Although we can measure the free energy of a system in thermodynamic equilibrium, typically all we can say about the free energy of a non-equilibrium ensemble is that it is larger than that of the same system at equilibrium. We have derived a formally exact expression for the probability distribution of a driven system. From this we find a simple near-equilibrium approximation for the free energy in

terms of an excess mean time-reversed work, which can be experimentally measured on real systems. 
Engineering environmental sensitivity in an artificial cell Principle Investigator: Daniel A Fletcher

\section{Project Description}

This project develops a system for studying in vitro expression of sensory proteins using a giant unilamellar vesicle platform. Biological systems are exquisitely sensitive to their local environments, using information such as osmolarity, light intensity, and $\mathrm{pH}$ to dynamically alter their behavior. In contrast, non-biological systems can have unique chemical, electrical, and physical properties, such as those found in nanostructured materials, but they are notoriously unresponsive in organization and behavior to their local environments. For applications ranging from development of adaptive materials to directed self-assembly of nanoparticles, a method for harnessing the environmental sensitivity of biological systems in a platform that is compatible with non-biological materials is needed. In this project, we propose to develop such a platform by reconstituting an environmentally sensitive two-component signaling system in synthetic vesicles using a newly developed encapsulation technology. Working with the E. coli osmolarity sensor EnvZ-OmpR as our test case, we will demonstrate the ability of a synthetic assembly to sense and respond to external conditions, setting the stage for the creation of responsive hybrid materials based on engineered gene expression.

\section{Accomplishments}

We accomplished two major goals during the first year of this project by (i) making a high-throughput version of our microfluidic encapsulation system for making unilamellar vesicles and (ii) demonstrating protein expression within the vesicles. We were able to increase the throughput of vesicle encapsulation by replacing the linear piezoelectric actuator in our original design with the cylindrical actuator of a 'droplet on demand' inkjet system. The use of the inkjet greatly increases throughput and size control compared to our initial device that demonstrated microfluidic encapsulation. We showed that multiple high-frequency pulses of the inkjet can be used to create microfluidic jets that deform a bilayer lipid membrane and form individual vesicles at high production rates, up to $200 \mathrm{~Hz}$. Variations in inkjet parameters including pulse number and voltage are used to control vesicle size. Further, by varying these parameters in combination with fluid viscosity, vesicle diameter can be varied over a wide range, $10-400 \mu \mathrm{m}$, including the size of many living cells.

For our first demonstration of protein expression inside of the unilamellar vesicles, we used green fluorescent protein (GFP). E. coli extract was loaded into the nozzle of the inkjet, which was surrounded by an iso-osmotic solution containing a plasmid with the DNA template for GFP. When the inkjet was fired to generate and load the vesicle, the plasmid containing GFP was entrained by the vortex ring created by the jet of extract so that both the plasmid and extract were encapsulated within the vesicle. Over time, robust GFP expression was observed, demonstrating that genes can be expressed in this "artificial cell" system. 


\section{Applications of Hybrid Live Cell - Synthetic Devices for Cancer Research Principal Investigator(s): Jay T. Groves}

\section{Project Description}

The purpose of this project is to utilize the hybrid live cell - supported membrane platform to reconstitute numerous functional aspects of cancer. In particular, the EphA2 receptor tyrosine kinase is overexpressed and functionally altered in $40 \%$ of human breast cancers, and recent evidence indicates that treatment with the ephrin-A1 ligand can reverse tumorigenesis. Since both Eph receptors and their ephrin ligands are natively membrane-bound, this signaling system is ideally suited to reconstitution into the hybrid live cell-supported membrane configuration.

We will first develop a display strategy to present laterally mobile ephrin-A1 on the surface of a supported membrane to EphA2-expressing breast cancer cells. Then we can study how the lateral organization, cluster size and mobility of ephrin-A1 ligands affect EphA2 signaling. These are all attributes of ligand presentation that exist in vivo, and the supported membrane platform allows us to access them in vitro. We can then examine correlations in EphA2 signaling across a panel of human breast cancer cell lines and relate changes in the molecular mechanisms of signaling to disease characteristics across the entire cell line library. In this way, controlled in vitro experiments on live cultured cells can be related to disease pathology in humans.

\section{Accomplishments}

The core preliminary observation that large-scale spatial reorganization of the EphA2 receptor occurs concomitantly with ephrin-A1 ligand triggering has been confirmed. More importantly, we have confirmed that mechanical restriction of EphA2 transport, using nanofabricated barriers to transport on the supported membrane surface, materially alters signaling outcome, in terms of metalloprotease recruitment and cytoskeleton morphology.

To probe the generality of this spatial reorganization phenotype, we measured EphA2 transport across a library of mammary epithelial cell lines. We found that there was a strong correlation between EphA2 translocation and the ability of a cell to invade foreign tissue. A system-wide correlation of EphA2 transport scores with all available protein and gene expression data across this library revealed several genes and proteins that are associated with this phenotype which could serve as therapeutic targets for receptor reorganization.

We have already obtained some follow-on funding to extend limited portions of this work, including probe of specific mechanical aspects of signaling in cancer, from the National Cancer Institute and the Department of Defense Breast Cancer Research. 


\title{
Light-boosted fermentation in the yeast Saccharomyces cerevisiae: Towards a science of cell architecture
}

\author{
PI: Jan Liphardt, Physical Biosciences Div. Co-PI: Manfred Auer, Life Sciences Div.
}

\section{Project Description.}

In the long term, transportation fuels may be synthesized on industrial scales with highly customized living cells. These cells will be constructed using synthetic biology approaches and will be fine-tuned to perform a small number of precisely defined reactions with great efficiency. Examples may include cells that convert $\mathrm{CO}_{2}$ and sunlight into biofuels.

To realize this prediction, it will be essential to discover and develop general principles that will ultimately give synthetic biologists control of cell shape and ultrastructure. Consider the photoreceptor cells in the human eye. There, the plasma membrane is folded back on itself numerous times. This "membrane shelving" increases the surface area of the cell membrane, and therefore, the capture cross-section for photons. Without this special shape of the cell, the human eye would be hundreds of times less sensitive to light, and we could not see at dawn or dusk. This is one of many examples where the shape or ultrastructure of a cell greatly enhances the function of an enzyme, such as the Rhodopsin protein in our photoreceptors.

\section{Accomplishments.}

In previous work (FY 2008), we created a strain of S. cerevisiae (Baker's yeast) that features extensive internal light-collecting structures. S. cerevisiae does not normally harvest light energy, but it is an efficient producer of ethanol and therefore a good model system to study conversion of glucose into biofuels or biofuel precursors. To our knowledge, this is the first time that a eukaryotic cell has been re-engineered to contain a multilayer membrane structure similar to the photoreceptors in our eyes or the chloroplasts in plants. As can be seen in the figure, proteorhodopsin (PR) constructs containing a nonmonomeric GFP tag form semicircular membrane aggregates which can be seen both in fluorescence and electron microscopy. By influencing membrane structure and creating membrane shelves, the proteorhodopsin-GFP construct transforms certain organelles of this heterologous organism, S. cerevisiae, in precisely the right way to theoretically maximize its proton-pumping efficiency.

Our major focus in FY 2009 was to dissect the ability of certain PR derivates to reprogram internal membrane architecture. We speculated that protein-dimerization (or oligomerization) was responsible for stacking. We tested this prediction in three ways. First, we replaced the nonmonomeric GFP with a strictly monomeric GFP. As hoped, the membrane stacking dissapeared. Second, we appended to the PR a small-molecule dimerizer domain. These domains dimerize in the presence of a small molecule, added to the growth medium. Strikingly, we discovered that upon addition of the small molecule, the membranes did not form stacks, but instead, formed a completely unexpected "bundle of grapes" architecture. Third, we added lysine zippers to the PR. These lysine zippers feature tunable self-affinity. The idea is to relate self-affinity to membrane morphology. These experients are currently underway.

Overall, we are learning that it is possible to control membrane architecure in ways that are central for bioenergy applications, but it is also clear that we are far from a fundamental mechanistic understanding of how certain proteins control membrane architecture. 


\title{
LB09033
}

\author{
The Control of Intraflagellar Transport in Chlamydomonas Cells \\ Principal Investigator(s): Ahmet Yildiz
}

Project Description

The purpose of this project is to observe how microtubule motors interact with each other and how cells control the activity of these motors to coordinate bidirectional transport. We use Chlamydomonas IFT as a model system and apply single molecule imaging techniques to address these questions. These studies will enable us to dissect how many motors carry a single cargo in IFT and whether opposite polarity motor proteins, kinesin and dynein, coordinate with each other or compete in a tug-of-war manner during the transport. The data will further enable to solve the mechanism by which cargoes travel long distances, reverse their direction, and the exchange of motors at the turnaround zones. Using genetically modified strains and superresolution imaging in live cells, we aim to discover the enzymes that play a role in regulation of motor protein activity. These assays will present new insight on molecular mechanism of intracellular cargo transport in general and provide fresh insight on several types of human diseases.

We develop novel experimental geometry that can test several predictions about the control and regulation of IFT. Paralyzed flagella $(p f)$ mutants of Chlamydomonas cells were rigidly immobilized onto a polylysine coated glass coverslip. Fluorescent beads coated with a monoclonal antibody against a flagellar membrane glycoprotein (FMG1) were then flowed into a glass chamber. The beads bind to the exterior region of FMG1 transmembrane protein and linked with motor proteins moving along the flagellum. Beads were moved either anterogradely or retrogradely at rates that are in agreement with the speed of the IFT particle, suggesting that beads are driven by IFT.

Accomplishments:

Our most significant accomplishment has been to track cargo movement along rigidly immobilized flagella by ultrahigh spatial $(\sim 1 \mathrm{~nm}$ error) and temporal $(600 \mu \mathrm{sec})$ resolution. We have observed $8 \mathrm{~nm}$ stepping towards both ends, in agreement with in vitro measurements of kinesin and dynein step sizes. Movement of the beads was unidirectional over distances longer than a micron. The bead usually reverses its direction at the tip of the flagellum. Remarkably, just before the reversal of the cargo, we observed high fluctuations in position for $\sim 100 \mathrm{msec}$. The cargo then abruptly starts moving in the opposite direction without showing any forward-backward stepping. These results indicate that IFT differs significantly from other bidirectional transport systems. The motors responsible of IFT can be switched on and off quickly at the turnaround zones so that they do not compete against each other to determine the direction of cargo transport.

We have also performed preliminary force measurements on single cargo transport. $1 \mu \mathrm{m}$ diameter latex beads were crosslinked to anti-FMG1 monoclonal antibodies at various concentrations and brought on top of a surface-immobilized flagellum by an optical trap. We have observed that the number of moving beads depends heavily on the amount of antibody on the bead surface. At low $(0.4 \mathrm{mg} / \mathrm{g}$ bead $)$ antibody concentration, beads are expected to attach to the flagellar membrane with a single antibody . Stall force measurements showed that most of the beads that are coated at high ( $>5 \mathrm{mg} / \mathrm{g}$ bead) antibody concentration escaped the trap, indicating that they generate more than $45 \mathrm{pN}$ of force. Beads with low $(0.4 \mathrm{mg} / \mathrm{g}$ bead $)$ antibody concentration showed much smaller forces of $10-30 \mathrm{pN}$. These results suggest that, single cargoes are driven by two or three motors on average along each direction.

To resolve the underlying mechanism of recycling kinesin and dynein motors in IFT, we are in the process of introducing CFP gene into dynein light intermediate chain. This strain will then be mated with GFP-tagged alleles of KAP subunit of kinesin. We will perform single molecule fluorescence tracking of motor proteins to observe whether they are attached to the same cargo along the bidirectional motion. 


\author{
Advanced Si Detectors for Future Short Pulse X-ray Sources
}

\author{
P.I.: Marco Battaglia and Peter Denes
}

\title{
Project Description
}

The development of future X-ray sources, high energy electron accelerators, as well as accelerator for hadrontherapy, requires detectors of new design to provide efficient beam monitoring and diagnostics. Requirements involve both operation in very high particle fluence for the primary beam monitoring and high precision and fast readout detection of soft particles. The short, intense pulses which they generate are difficult to image with conventional methods. We develop new pixellated Si sensors based on innovative semiconductor technologies and apply them in experiments under realistic conditions at the ALS and LOASIS.

\section{Accomplishments}

The two main accomplishments of the project during FY09 are i) the design, production and test of a Silicon-On-Insulator (SOI) pixel sensor able to operate at depletion voltages up to $50 \mathrm{~V}$ and ii) the deployment of a calibrated CCD system for X-ray spectroscopy on the LOASIS beam line.

SOI pixels sensors designed and produced as part of this LDRD program have been tested on the 1.5 $\mathrm{GeV}$ electron beam of the ALS-BTS beam line obtaining $\mathrm{S} / \mathrm{N}=20$ for a $50 \mathrm{MHz}$ readout. The latest chip, produced in OKI 0.2 micron SOI-FD technology have been operated in the lab with depletion voltages up to $50 \mathrm{~V}$. The evolution of the signal obtained from a highly collimated IR laser spot with the applied depletion voltage demonstrates that the sensor sensitive layer increases according to the expected $\ulcorner\mathrm{V}$. This result opens new perspectives in the application of SOI pixel sensors to the detection of soft X-rays and electrons, topical to this LDRD project. In parallel with the sensor development an R\&D program on thin SOI sensor backplane post-processing to create an ultra-thin entrance window has been started. Two processes are being explored. The first is a low-temperature phosphor implant performed by the group of Craig Tindall in the Engineering Division at LBNL and the second the delta-doping process developed by the Advanced Detector \& Nano Science Technologies group at JPL-NASA. Test SOI chips have been processed with the LBNL phosphor implant and have been tested to be fully functional after the backplane post-processing. In parallel SOI chip backthinning to 100 and 50 micron has been successfully carried out at JPL-NASA.

While the SOI pixel development aims at very fast sensors with on-chip data processing capabilities, beam diagnostics applications also require high energy resolution and the exposure to large fluxes with minimal pile-up. In order to evaluate the operating conditions on the test case application chosen by this LDRD project, we have carried out a first experiment using a thick, high-resistivity CCD, developed at LBNL for application in SNAP/JDEM, at LOASIS. The CCD sensor has been calibrated at the ALS on beam line 5.3.1 and subsequently deployed at LOASIS for data taking in August 2009. The experiment consisted in the study of the spectrum of betatron X-rays produced by electrons oscillating in the focusing field of the plasma. The CCD sensor is well suited since its high pixellisation allows us to isolate a pure sample of individual $\mathrm{X}$-rays despite the large electron and photon flux. The CCD high energy resolution ensures a precise spectrum reconstruction. In this experiment we have measured the spectrum of X-rays generated in the process of electron acceleration in the range of interest $2-20 \mathrm{keV}$. This is a first measurement of the energy of betatron X-ray emission over the full spectrum and the data demonstrate a correlation with the electron energy and the laser parameters, as expected. 


\section{Effective Theories for Collider Physics Principle Investigator: Christian Bauer}

\section{Project Description:}

The goal of the LDRD project was to increase our theoretical understanding of collider physics from the framework of effective field theories. It is well known that many aspects of the strong interaction of the standard model are too complicated to be calculated from first principles. Non-perturbative effects are important in precise predictions, and a clear separation of scales is important to separate the non-perturbative information from the perturbatively calculable physics. Effective field theories are the ideal tool to separate widely separating scales from one another, and are therefore ideally suited for this research goal. Several different applications of effective field theories to collider physics are investigated.

\section{Project Achievements:}

We have developed new factorization theorems for important observables such as event shape variables and generic jet observables. Members of my group are now using these techniques to study the sub-structure of jets, which will eventually allow distinguishing jets according to the parton it is created by.

A second aspect of my research has been to understand the origin of large logarithmic terms in the perturbative expressions of jets observables. Using soft-collinear effective theory, we showed that the leading logarithms arising in the production of $\mathrm{W}$ bosons in association with jets can be resummed to all orders by a suitable choice of the factorization scale, increasing the accuracy of previous calculations. Recently, we started to investigate the origin of threshold logarithms. While such logarithms have been resummed for some simple processes in the past, a formal understanding of the approximations made in the calculations has been lacking in the literature. We have shown how to perform an expansion in the steepness of the parton luminosities. A second part of this project is to extend the existing formalism to allow the resummation of threshold logarithms for events containing tagged jets.

Finally, a large effort of the project was to increase our understanding of the in merging of partonic calculations with parton shower algorithms. While simple techniques have been developed to combine leading order calculations with parton showers, only limited knowledge exists how to extend these results to higher orders in perturbation theory. Using the event generator GenEvA, developed as part of the LDRD project, we have recently developed the necessary framework for the extension to next-to-leading order calculations. We are currently finishing the implementation of this framework into GenEvA, and expect results soon. 


\section{Development of Nanowire Carpet Hybrid Pixel Detectors Principal Investigator(s): Maurice Garcia-Sciveres}

\section{Project Description:}

The purpose of this project is to produce and test demonstration prototypes of a new fabrication process for silicon pixel radiation detectors. So called hybrid pixel detectors are currently fabricated by lithographically processing silicon sensor wafers, which is done by a variety of commercial providers. To produce a working detector, the lithographically produced sensors must be electrically connected to custom integrated circuits, which is currently done using fine pitch flip-chip bump bonding. By way of this new process, lithographically produced sensors are replaced with a silicon wafer that has been provided with a carpet of nano-wires covering the interface surface, these nano-wires making electrical contact with the readout chip. This work involves treating and then etching the surface of a special silicon wafer to produce electrical characteristics appropriate for radiation detection, followed by the investigation of methods to attach this new type of sensor to the readout chip. If successful, this process would enable lower cost hybrid pixel detectors by eliminating both of the presently cost-driving steps of sensor lithographic processing and fine pitch bump bonding.

This work was supported by LDRD funding and benefited from access to Molecular Foundry (MF) equipment and advice through a MF instrument proposal. The nano-fabrication techniques used are not new and have been learned from MF staff and from published literature. The novelty of this work lies in the application of nano-fabrication techniques to the production of working instruments that can currently only be produced by less efficient and therefore more costly methods.

\section{FY09 Accomplishments:}

FY09 was the final year of this LDRD. Using a fabrication recipe developed in FY08, a standard sample format was defined and of order 10 samples were fabricated to enable a test and measurement program to continue into FY10 (beyond the end of LDRD support). These samples include the new features of back-side metallization and a front-side guard ring structure to control edge leakage currents during operation as a radiation sensor. In addition to the nanowire samples, existing readout integrated circuits were prepared with indium metal pads to contact the nanowire carpet. These samples and readout ICs were used for a measurement program carried out with a SULI undergraduate student during Summer FY09 ( E. Sprague) and continuing with a UCB URAP undergraduate student during FY10 (D. Nguyen). Current vs. voltage and temperature behavior characteristic of a radiation detector has been demonstrated in FY09. Testing with radioactive sources is in progress in FY10.

A provisional patent application was filed in October 2008, allowing up to 1 year for a full patent to be filed, if deemed appropriate. After an evaluation of the market potential by the LBNL patent office, the decision was made not to file. The potential market for this kind of radiation detector was considered too small to justify the expense of an international patent, which would needed because all manufacturers of traditional pixel radiation detectors are overseas. The decision not to file lifted any restriction on public presentation of technical details. A journal paper or conference submission is planned for FY10. 
Development of Multi-Modular Assemblies with Reduced Material and Services for Tracking at Future Colliders

Principal Investigator(s): M. Gilchriese and C. Haber

\section{Project Description}

Future silicon tracking detectors for the Super Large Hadron Collider (SLHC) require the development of multi-modular mechanical support structures with integrated cooling and electronics on an unprecedented scale. The purpose of this project is to study a concept which can meet the requirements of such tracking systems. The concept is based upon thin, low mass, stiff composite laminated structures which embed both high performance cooling and electrical distribution of high bandwidth signals and efficient power distribution methods.

The project consists of two principal aspects. One of these is the design of integrated mechanical/cooling structures that could be applicable to all elements and regions of a future silicon tracking system for the SLHC. These require both design studies (analytical calculations and finite-element calculations) and the fabrication of a number of prototypes to explore and test the design concepts. The second aspect is to assemble and test the electrical performance of prototype integrated structures using silicon strip modules.

Accomplishments

We completed conceptual designs for mechanical/cooling structures for all regions of a future pixel silicon tracker based on a common design principle - the use of low-density, thermal conducting carbon foam (a new material) coupled with readily available carbon fiber materials and metallic cooling tubes. A program of construction of prototypes was initiated and partially completed. A preliminary verification of the thermal performance of the concept was completed. This included stress testing (thermal cycling to $-35 \mathrm{C}^{\circ}$ or below, including using liquid nitrogen), testing with water (in turbulent flow) and evaporative $\mathrm{CO}_{2}$ cooling and mechanical distortion measurements. The use of a new type of carbon, thermally conducting foam continues to show great promise. Preliminary tests indicate that design requirements can be met.

In the prior year work period we reported on the fabrication and test of a multi-modular electrical stave structure and test have continued over the last year. We demonstrated a large scale application of serial powering, with good electrical performance. In the serial powering test we showed that at least 30 electrical drops can be operated with stability. In order to embed the multi-module system in a practical configuration various aspects of data transmission, interface, and multiplexing must be demonstrated. In collaboration with SLAC and UK colleagues an interface chip was developed. We developed a test system and packaging arrangements to demonstrate the performance of this device and test large numbers. We also initiated the design of an interface circuit to connect to the external DAQ, control, and powering. A number of new fine pitch bus cable structures were designed and fabricated. A key issue is to be able to glue these structures down in a uniform way. This was studied and improvements made. Multi-drop data transmission was demonstrated on bus cables as well. A large test structure with 24 drops was assembled with particular variations imposed on the clock receiver circuits to facilitate multi-drop AC coupled operation. The development of high bandwidth data acquisition and control was also an area of significant activity during this period. In collaboration with SLAC and UK groups a new FPGA based platform was developed. We wrote firmware and software to operate this system and demonstrated it on hybrids from the earlier stave development. 


\section{LB08046}

Calibrating Baryon Oscillations for Future Experiments PI(s): Nikhil Padmanabhan, Martin White, David Schlegel

Project Description: The baryon oscillation method is now an essential element of all programs to measure the expansion rate of the Universe and constrain the properties of "dark energy". The next generation of surveys will constrain the expansion to $1 \%$ at multiple redshifts and the Joint Dark Energy Mission (JDEM), a DOE-NASA mission that will achieve $\sim 0.1 \%$ constraints, is already being planned. This project provides essential the theoretical support for these experiments, by quantifying possible systematic errors and developing methods for optimally extracting the baryon oscillation signal from these data sets. We aim to describe the effects of the (a) nonlinear clustering of the dark matter, (b) halos and galaxies, and (c) redshift space distortions on the acoustic feature. We do this with large numbers of N-body simulations, varying simulation resolution and cosmology to ensure the robustness of our results. Combining these simulations with perturbative techniques, we aim to develop an analytical framework that captures the above effects at the fidelity required by the next generation of surveys. At the same time, we plan on developing new analysis techniques that could reduce these effects and quantify how such methods might drive the design of the next generation of surveys.

Accomplishments: We have made significant progress on understanding how the nonlinear clustering and halo bias affect the baryon acoustic feature. We have run $\sim 1,000 \mathrm{Gpc}^{3}$ of $\mathrm{N}$ - body simulations (the next generation of experiments will survey $\sim 10 \mathrm{Gpc}^{3}$ of the Universe), across four very different cosmologies and for a variety of simulation parameters. We demonstrated that our current simulation techniques are accurate enough to correctly model the baryon oscillations and used these simulations to demonstrate that the effect of nonlinear clustering on the baryon oscillations is accurately described by second-order (1-loop) perturbation theory for both biased and unbiased tracers. This allowed us to construct a analytic model for baryon oscillations in the nonlinear regime that is accurate to the $\sim 0.1 \%$ level necessary for future surveys. We additionally quantified the requirements of "reconstruction" methods (designed to undo some of the effects of nonlinear clustering) on the survey parameters, which has important implications when optimizing the design for the next generation of surveys. Preliminary investigations of acoustic oscillations in the intergalactic medium were reported, a potentially game changing technique. In addition, we have improved our analytic understanding of how these methods work and what their regimes of validity are. These efforts have suggested improvements that can be made to such methods, an area for future research (with NASA funding now in hand). They have also allowed us to focus our simulation resources on the most challenging areas while using the analytic methods where they are applicable. This makes us more efficient and has had spin-offs beyond just this project, since simulations are now integral to most dark energy experiments/methods. 


\section{R\&D for Fast, Low-Noise CCD Readout and Single Photon Detection Capability}

Natalie Roe, Physics Division

Peter Denes and Steve Holland, Engineering Division

Project Description: We proposed an R\&D project aimed at designing advanced CCDs, based on the current state-of-the art fully-depleted p-channel CCD developed at the LBNL Microsystems Laboratory (MSL). In FY09 we proposed to design CCDs capable of singlephoton detection, lower energy detection, faster readout, and better noise performance and drive capability. Applications of these technologies include the next generation of ground and space based astronomical surveys to study dark energy, x-ray detection at the ALS, LCLS and other photon sources, and night vision and radiological surveillance for homeland security applications.

Accomplishments: Twelve new CCDs have been designed on a six-inch wafer layout that includes a total of $28 \mathrm{CCDs}$ (some are controls based on previous designs and others are duplicates). The designs include the following devices:

1) Two-stage output amplifier for fast readout and higher drive capability

2) Small (1 um) contacts to channel stops to improve persistent image mitigation

3) Buried contact amplifiers to reduce capacitance and improve read noise

4) Same as 3) but with smaller buried contacts

5) Floating gate amplifiers to enable non-destructive charge sensing

6) Segmented large format CCD for next generation dark energy survey

7) Same as 5) but with variation in the readout architecture

8) Charge multiplying CCD for single photon detection

9) Same as 8) but with new architecture for multiplication cell

10) Fast CCD in large format with multiple readouts and frame-store capability for ALS

11) Same as 10) but in smaller format, no frame-store

12) Same as 11 but with missing pixels to provide for beam passage through the middle

The CCD wafer design was completed in July 2009. After a month of design and layout checking the design was completed in August 2009. The next and final year of the project will cover the fabrication and testing costs of the new devices. 


\title{
Baryon Oscillations and Dark Energy: Prototyping Instruments
}

\author{
Principal Investigator: David Schlegel
}

\section{Project Description}

Future baryon acoustic oscillation (BAO) experiments for measuring dark energy witll require technologies to control more than 1000 fibers on the focal plane of a telescope. A simple, cost-effective approach is to individually actuate each fiber in the focal plane. This removes the complication and expense of drilling plug plates (like for the Sloan Digital Sky Survey) or building robots to move fibers meter distances with the required $30 \mu \mathrm{m}$ tolerance.

\section{Accomplishments}

This final year of LDRD was promised to "complete this prototype to the level required for large instrument proposals." That work was completed to the point required for the white papers of the ambitious BigBOSS project.

The initial prototype of the LBNL fiber actuator was completed last year. That actuator was scaled to a spacing of $19.2 \mathrm{~mm}$ center-to-center. In this final year of LDRD, the actuator design was modified in scale for the BigBOSS proposal. The spacing in the new design is 11 $\mathrm{mm}$ center-to-center, making use of MicroMo $6 \mathrm{~mm}$ motors.

BigBOSS is a project developed in 2008-2009, proposing to build a ground-based instrument to accomplish the Stage IV BAO dark energy experiment. A white paper describing this proposal was developed with the JDEM scientists and technical staff at LBNL. The project relies upon the fiber positioning technology developed in this LDRD. The BigBOSS team submitted a white paper to the National Academy Decadal Survey in April 2009, and a white paper to the DOE Particle Astrophysics Scientific Assessment Group (PASAG). We are building an international collaboration to realize this proposal. 


\section{Decoding Dark Energy with Weak Gravitational Lensing \\ Principal Investigators: George Smoot, Alexie Leauthaud}

Project Description

Understanding the nature of dark matter and dark energy represents two outstanding challenges for the new century. Whereas compelling evidence suggests that dark matter is non-relativistic and collisionless, thus guiding our quest for the appropriate particle, the general properties of dark energy remain far more elusive. We propose a comprehensive study of weak lensing as one of the most powerful means to study dark energy. We will carry out this study in two steps: first by analyzing the ability of weak lensing to directly detect massive clusters and second, by comparing these results to various other weak lensing statistics that are also sensitive to the dark energy equation of state.

Accomplishments

Firstly, in 2009 we have completed a study of the weak lensing signal around groups and clusters in the COSMOS field (see Figure 1). Groups have been stacked according to their X-ray Luminosity $\left(L_{X}\right)$ which allows for a better understanding of the relationship between the hot intra-cluster gas and dark matter halos. This study is an important step forward towards better measurements of the abundance of galaxy clusters as a function of halo mass. These results have been accepted to the Astrophysical Journal. Building on this carefully calibrated scaling relation, we have started a study of the abundance of clusters and groups within the COSMOS field to check if our results are in agreement with the current cosmological model.

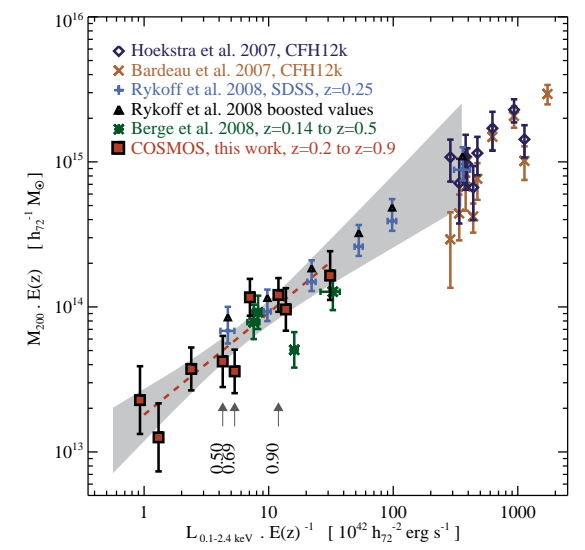

Figure 1: Figure: the relationship between X-ray Luminosity and Halo mass as measured by weak gravitational lensing in the COSMOS survey (Leauthaud et al. 2009 , in press)).

Secondly, in 2009, we have also constructed a novel method by which to correct imaging data for the undesirable effects of Charge Transfer Efficiency which can affect the measurement of galaxy shapes. This is an important step forward towards designing future space-based weak lensing mission (Rhodes et al. 2009 submitted, Massey et al. 2009 in press). 


\section{Cross-Divisional}

\section{Integrated Tools in Multiscale Imaging}

PI: Manfred Auer (Life Sciences Division) co-PI: Jan Liphardt (Physical Biosciences Division) Phil Hugenholtz (Joint Genome Institute),Terumi Kohwi-Shigematsu (Life Sciences Division) Project description.

The overall goal of this project is to invent and apply a new generation of imaging technologies to biological systems central to DOE's mission. This includes characterizing living biological systems in physiological conditions, ideally, inside living tissues and biofilms and other microbial communities. To that end, we are pursuing an integrated three-part research and development strategy focusing on hardware, algorithms, and chemistry. Our biological test systems are (1) lignocellulose degradation and (2) low-dose biology.

The termite hindgut microbial community is one of nature's most efficient lignocellulose biodegrading "factories". Metagenomic analysis has revealed that the hindgut microbial community is composed of about two hundred microbial species. We seek a fundamental understanding of how community spatial organization and composition affects lignocellulose degradation using 2D wide-field transmission electron microscopy (TEM) as well as 3D focused ion beam scanning electron microscopy (FIB/SEM) imaging. Structural analysis is complemented by $16 \mathrm{~S}$ rRNA fluorescence in-situ hybridization (FISH) labeling and correlative light and electron microscopy.

The SatB1 protein is a chromatin organizer that regulates genes by looping chromatin. SatB1 is a central participant in the cellular response to low-dose ionizing radiation and, like many other factors involved in DNA repair, also has been implicated in human diseases such as breast cancer. SatB1 is currently the only protein known to organize the genome by tethering its target genes onto its cage structure. Once genes are recruited to the SatB1 cage, it serves as a platform for assembly of chromatin remodeling/modifying factors, which in turn establishes the specific epigenetic status of these loci.

\section{Accomplishments:}

For termite hindgut studies, we have found that plant biomass in the hindgut is colonized predominantly by two morphological types of bacteria that utilize different strategies of cell wall adhesion and degradation: (1) intimate cell-substrate contact or (2) by secretion of (presumably enzyme-laden) vesicles. We have also succeeded in developing correlative FISH/TEM imaging of resin-embedded sections. This allows us, for the first time, to identify selected community members by FISH and to simultaneously visualize their roles in biomass degradation. To our surprise we have found that one type of microbe may be responsible for much of the lignocellulotic activity of the hindgut. It appears to function by enzymatically drilling narrow channels into the plant biomass cell wall. Our next major milestone will be to identify this unknown "super-degrader" microbe and its macromolecular inventory. Enzymes secreted from such microbes may hold the key for effective commercial lignocellulosic biofuel production.

For the SatB1 protein studies, by simultaneously refining imaging hardware (reducing thermal drift), algorithms (to generate 3D nanometer precision maps), and chemistry (sample fixation protocols), we have succeeded in generating the first-ever nanometer precision maps of how SatB1 is organized in the mammalian nucleus, a critical requirement if we are to understand its ability to function as a 'master regulator' of gene expression. In parallel work, we have also developed a strategy for reliable and specific delivery of quantum dots to the mammalian nucleus, which allows us to follow proteins as they move inside the nucleus and bind DNA. Together, the ability to create nanometer precision maps of DNA binding proteins, as well as watch complexes assemble and move inside the nucleus, will greatly facilitate DOE's overall goal of a mechanistic understanding of DNA repair and low-dose radiation biology. 
Fabrication of photovoltaic devices using genetically engineered biomaterials Principal Investigator(s): Seung-Wuk Lee

\section{Project Description}

The purpose of this project is to fabricate virus-based photovoltaic devices to harness novel and clean solar energy. The monodisperse photovoltaic semiconductor nanowires will be synthesized through a mineralization process on genetically engineered viruses. Photovoltaic devices will be fabricated using semiconductor covered-virus nanowires through a conventional organic-inorganic hetero junction photovoltaic solar cell fabrication process. The approach will exploit combinatorial phage display to identify specific binding peptide information against photovoltaic semiconductor materials $\left(\mathrm{CdTe}, \mathrm{ZnO}, \mathrm{TiO}_{2}\right.$, etc), which will direct mineralization of such materials in mild aqueous conditions. Using this tactic, we will engineer monodisperse viral particles to template photovoltaic semiconducting materials. These viruses will then be vertically self-assembled on transparent electrodes to fabricate solar cell devices.

We will develop virus-based organic-inorganic hetero junction photovoltaic devices, by exploiting viruses engineered to have specific affinity between a coat protein and photovoltaic semiconducting nanomaterials. The biological approach to build the proposed photovoltaic solar cell materials and devices has many promising aspects: 1) we can isolate previously unexplored novel peptide information against photovoltaic semiconducting material. Using the identified peptide information, we can design novel organisms, microbes, and biomaterials, with which we can open new fields of synthetic biology and couple many conventional micro-fabrication approaches (such as, soft lithography, DPN lithography, microprinting, and microfluidics) to build novel devices through bottom up processes.

2) Using recombinant DNA techniques, we modify the virus and subsequent nanowire scales, scales, which is suitable for the optimized device fabrication. In addition, cloning nature of the biological materials can simplify preparation of the sample through a self-replicating amplification processes. 3) Due to the diamagnetic property of the virus, we can align them between the electrodes using external magnetic filed in the hetero junction devices, which is expected to enhance the efficiency of the photovoltaic devices by improving electron mobility. 4) Obtaining knowledge between biomolecules and electronic materials is critical for the design of novel nanoscale bio-electronic devices. 5) This multifaceted research project will provide graduate and undergraduate students with many opportunities to explore, integrate and educate the next generation of scientists in a variety of diverse research disciplines.

\section{Accomplishments}

We successfully constructed long range ordered liquid crystalline films to fabricate the novel photovoltaic energy producing materials and studied characterization of the photovoltaic efficiency of the proposed materials. In addition, due to the inherent biological structure of the coat protein, the virus was expected to possesses piezoelectric properties. We engineered the coat protein of the virus and fabricated a multi-virus structure that could convert electric energy to mechanical energy and mechanical energy to electric energy. The piezoelectric coefficient of the multi-virus structure was measured. 


\title{
Coupled Process Models, Separations, Monitoring for Advanced Nuclear Fuel Cycles
}

\author{
Principal Investigator(s): Andrew Canning, Phil Collela, Per Peterson, Lee Schroeder, \\ Jim Siegrist, David Shuh, Niels Jensen (UC Davis)
}

\section{Project Description:}

Advanced nuclear fuel cycles require improved modeling and performance monitoring tools to optimize the fuel cycle, reactor design and licensing, and waste storage and disposal. This project will develop next-generation simulation models for reactor elements and licensing, model fast fuel cycle actinide behavior and develop instrumentation for the non-invasive study of waste forms and related radioactive materials critical to the nation's nonproliferation and safeguards activities.

\section{Accomplishments:}

Work, extending the Chombo Adaptive Mesh Refinement framework, on heat transfer between solid materials with irregular interfaces concluded with a successful test of the code on a nineteen-pin reactor fuel bundle. For a realistic sodium fast reactor we were able to efficiently solve the heat equation for reactor pins containing a heat source and a stationary moderator. Generation of the full pin geometry, including wire wrappings on each pin, was shown to occupy only a small portion of the simulation time. We have successfully implemented, and are currently testing, a solver for buoyant, incompressible flow in the Boussinesq approximation.

Completed an adaptable framework for the use of advanced simulation results in the USNRC General Design Certification process. The framework outlines general simulation requirements and was used to evaluate the use of granular flow simulation models for pebble-fueled reactor cores. Also implemented a granular flow code to study fuel zoning and mixing that allows easy implementation of different core geometries. Initial simulation results are in good qualitative agreement with scaled experiments.

Several new 2,6-Bis(2-benzimidazyl) pyridine (BBP) ligand complexes with different charges have been synthesized, crystal structures determined, and electronic structures characterized by soft x-ray near-edge x-ray absorption fine structure (NEXAFS) at the light element K-edges at the Advanced Light Source (ALS). These complexes serve as analogs for actinides for comparing bonding characteristics and represent the first direct probe of N-bonding for a series of isostructural (lanthanide) complexes. Complementing the experimental effort, theoretical work focused on understanding the actinide f-electron bonding in the new $\mathrm{U}$ and proposed $\mathrm{Pu}$ complexes with new ligands. First principle studies were performed for the BBP-type ligands containing $\mathrm{U}$. These calculations were aimed at determining the suitability of different ligands in the trivalent separation of actinides and lanthanides for advanced fuel cycles.

Nuclear resonance fluorescence techniques were employed to study their potential for nondestructive and quantitative isotope identification of spent nuclear fuel. A modeling and simulation study was carried out to evaluate this technique for its use in safeguards applications. Initial findings were encouraging and resulted in several proposals being funded by the Department of Energy. 


\section{Publications List}

AFRD-Sannibale et al LB09007 Experimental Accelerator R\&D Toward a Future Light Source

Refereed Publications

K. Baptiste, J. Corlett, S. Kwiatkowski, S. Lidia, J. Qiang, F. Sannibale, K. Sonnad, J. Staples, S. Virostek, R. Wells; "A CW normal-conductive RF gun for free electron laser and energy recovery linac applications," Nuclear Instruments \& Methods in Physics Research A 599, 9 (2009).

\section{Conference Proceedings \& Presentations}

K. Baptiste, J. Corlett, T. M. Huang, S. Kwiatkowski, D. Li, J. Qiang, F. Sannibale, J. Staples, R. Wells, L. Yang, A. Zholents; "Status of the LBNL Normal-Conducting CW VHF Photo-Injector," Proceedings of the 2009 Particle Accelerator Conference, Vancouver, Canada, May 2009.

K. Baptiste, J. Corlett, R. Kraft, S. Kwiatkowski, J. Qiang, F. Sannibale\#, J. Staples, R. Wells, L. Yang, A. Zholents ; "Status and plans for the LBNL normal-conducting CW VHF photo-injector," Proceedings of the 2009 Free Electron Laser Conference, Liverpool, U.K., August 2009.

F. Sannibale, Contributed talk, "Status and Plans for the LBNL Normal-Conducting CW VHF Photo-Injector," at the 2009 FEL conference, Liverpool, U.K., August 2009.

F. Sannibale. Seminar, "The LBNL CW normal-conducting RF photo-injector," Brookhaven national Laboratory, Upton, NY, USA.

AFRD-Schenkel LB08017 Quantum Information Science with Integrated Color Centers in Diamond

C. D. Weis, A. Schuh, A. Batra, A. Persaud, I. W. Rangelow, J. Bokor, C. C. Lo, S. Cabrini, E. Sideras-Haddad, G. D. Fuchs, R. Hanson, D. D. Awschalom, and T. Schenkel; "Single-atom doping for quantum device development in diamond and silicon", J. Vac. Sci. Techn. B 26, 2596 (2008).

AFRD-Toth LB08022 Experimental Demonstration of a Laser-Plasma-Accelerator Driven Free-Electron Laser

\section{Refereed Publications}

F. Gruener, C. B. Schroeder, A. R. Maier, S. Becker, J. M. Mikhailova; "Space-charge effects in ultra-high current electron bunches generated by laser-plasma accelerators," Phys. Rev. ST Accel. Beams 12, 020701 (2009).

\section{Conference Proceedings}

C. B. Schroeder, W. M. Fawley, F. Gruner, M. Bakeman, K. Nakamura, K. E. Robinson, Cs. Toth, E. Esarey, and W. P. Leemans; "Free-Electron Laser Driven by the LBNL LaserPlasma Accelerator," in Advanced Accelerator Concepts 2008, AIP Proceedings 1086, 637-642 (2009).

M. S. Bakeman, C. B. Schroeder, K. E. Robinson, Cs. Toth, K. Nakamura, W. M. Fawley, and W. P. Leemans; "Magnetic Characterization and Design of an Undulator-Based Electron Beam Diagnostic," in Advanced Accelerator Concepts 2008, AIP Proceedings 1086, 643-648 (2009). 
D. Panasenko, A. J. Shu, C. B. Schroeder, A. J. Gonsalves, K. Nakamura, N.H. Matlis, E. Cormier-Michel, G. Plateau, C. Lin, C. Toth, C. G. R. Geddes, E. Esarey, and W. P. Leemans, "Staging Laser Plasma Accelerators for Increased Beam Energy," in Advanced Accelerator Concepts 2008, AIP Proceedings 1086, 215-220 (2009).

Cs. Toth, O. Albert, L. Canova, A. J. Gonsalves, D. Panasenko, N. H. Matlis, G. Plateau, and W. P. Leemans; "Contrast Enhancement of the LOASIS CPA Laser and Effects on Electron Beam Performance of LWFA," in Advanced Accelerator Concepts 2008, AIP Proceedings 1086, 270-275 (2009).

M.S. Bakeman, W.M. Fawley, W. Leemans, K. Nakamura, K.E. Robinson, C.B. Schroeder, C. Toth; "Undulator-Based Laser Wakefield Accelerator Electron Beam Diagnostic," $P A C 2009$ (in press); url: www.jacow.org.

D. Panasenko, A. J. Gonsalves, W. Leemans, K. Nakamura, C. B. Schroeder, A. Shu, Cs. Toth; "Development of Water Jet Plasma Mirror for Staging of Laser Plasma Accelerators," PAC 2009 (in press); url: www.jacow.org.

\section{Invited Talks}

W.P. Leemans, Invited speaker, ICUIL - 2008, Tongli, China, October 28, 2008.

E. Esarey, Invited Seminar, Shanghai Synchrotron Radiation Facility, Shanghai, China, October 31, 2008.

W.P. Leemans, Invited Seminar, Shanghai Synchrotron Radiation Facility, Shanghai, China, October 31, 2008.

W.P. Leemans, Invited seminar, IHEP, Beijing, China, November 4, 2008.

W.P. Leemans, Invited seminar, Tsinghua University, China, November 5, 2008.

W.P. Leemans, Colloquium, Sacramento State Physics Department, November 13, 2008.

W.P. Leemans, Colloquium, MIT Physics Department, December 10, 2008.

W.P. Leemans, Colloquium, University of Hamburg, February 10, 2009.

W.P. Leemans, Colloquium, Stony Brook University, March 24, 2009.

W.P. Leemans, Colloquium, Caltech, April 2, 2009.

W.P. Leemans, Invited talk, Extreme Light Infrastructure conference, Paris, France, April 27-28, 2009.

W.P. Leemans, Invited talk, HEPAP meeting, Washington DC, May 21-22, 2009

W.P. Leemans, Invited talk, 39th Anomalous Absorption Conference, Bodega Bay, June 1419, 2009.

AFRD-Vay LB08023 Lorentz Compaction of Scales for Ultra-efficient Simulation of Advance Accelerators (and other systems)

J. -L. Vay, W. M. Fawley, C. G. Geddes, E. Cormier-Michel, D. P. Grote; "Speeding Up Simulations of Relativistic Systems using an Optimal Boosted Frame," Proc. DPF-2009, Detroit, MI (2009) url: arXiv:0909.5603 (Sept. 2009).

J.-L. Vay, W. M. Fawley, C. G. Geddes, E. Cormier-Michel, D. P. Grote; "Novel Methods for Simulating Relativistic Systems using an Optimal Boosted Frame," Proc. Int. Comput. Accel. Phys. Conf. Icap'09, San Francisco, CA (2009).

J.-L. Vay, W. M. Fawley, C. G. Geddes, E. Cormier-Michel, D. P. Grote; ”Application of the Reduction of Scale Range in a Lorentz Boosted Frame to the Numerical Simulation of Particle Acceleration Devices," Proc. Particle Accelerator Conference, Vancouver, Canada (2009) TU1PBI04. 
W. M. Fawley, J.-L. Vay; "Fully Electromagnetic Simulation of Free-Electron Laser Amplifier Physics via the Lorentz-Boosted Frame Approach," Proc. Particle Accelerator Conference, Vancouver, Canada (2009) WE5RFP029.

J.-L. Vay, D. L. Bruhwiler, C. G. R. Geddes, W. M. Fawley, S. F. Martins, J. R. Cary, E. Cormier-Michel, B. Cowan, R. A. Fonseca, M. A. Furman, W. Lu, W. B. Mori, L. O. Silva; "Simulating relativistic beam and plasma systems using an optimal boosted frame," Proc. SciDAC meeting, San Diego, CA (2009).

C. Huang et al, "Recent Results and Future Challenges for Large Scale Particle-In-Cell Simulations of Plasma-Based accelerator Concepts," Proc. SciDAC meeting, San Diego, CA (2009).

W. M. Fawley, J.-L. Vay; "Full 3D Electromagnetic Simulation of Coherent Synchrotron Radiation via the Lorentz-Boosted Frame Approach," APS'10, Portland, Oregon (2010, submitted abstract).

W. M. Fawley, J.-L. Vay; "title paper on FEL for PRSTAB," draft for submission to Phys.Rev.ST Accel. Beams.

J.-L. Vay, C. G. Geddes, E. Cormier-Michel, D. P. Grote; "Boosted Frame Modeling of 10 Gev Class Laser Wakefield Accelerator Stages," draft for submission to Phys.Rev.ST Accel. Beams.

J.-L. Vay, C. G. Geddes, E. Cormier-Michel, D. P. Grote; "Novel Numerical Techniques for Boosted Frame Modeling of Laser Wakefield Accelerators," draft for submission to $J$. Comput. Phys.

AFRD-Venturini, Zholents LB07034 Emittance Manipulation and Beam Conditioning for $\underline{\text { FEL'S }}$

E. Kur, G. Penn, J. Qiang, M. Venturini, R. Wells, and A. Zholents; “Accelerator Design Study for a Soft X-Ray Free Electron Laser at the Lawrence Berkeley National Laboratory," LBNL Report LBNL 2670-E, Berkeley (2009).

J. Corlett, K. Baptiste, J. Byrd, P. Denes, R. Falcone, J. Kirz, W. McCurdy, H. Padmore, G. Penn, J. Qiang, D. Robin, F. Sannibale, R. Schoenlein, J. Staples, C. Steier, M. Venturini, W. Wan, W. Wells, R. Wilcox, and A. Zholents; "Design Studies for a VUV Soft X-ray Free-Electron Laser Array," Synchrotron Radiation News 22, No.5 (2009).

A. Zholents, E. Kur, Ji Qiang, M. Venturini, and R. P. Wells; "Design of the electron beam switch yard for an array of Free Electron Lasers," CBP Tech Note 401 (March, 2009).

M. Venturini, M. Migliorati, C. Ronsivalle, and C. Vaccarezza; "Linear Gain for the Microbunching Instability in an RF Compressor," CBP Technical Note CB-TN-404 (2009).

C. Ronsivalle, M. Ferrario, C. Vaccarezza, M. Migliorati, and M. Venturini; "Microbunchig Studies for SPARX Photoinjector," PAC09 Proceedings (2009).

R. Bartolini, C. Christou, J.H. Han, I.P.S. Martin, J.H. Rowland, M.Venturini, D. AngalKalinin, F. Jackson, B. Muratori, and P. Williams; "Optimization of a singe-pass superconducting linac as a FEL driver for the NLS project," FEL 2009 Conference, Proceedings (2009).

S. Di Mitri, E. Allaria, L. Badano, et al; "Design and simulation challenges for FERMI@elettra," Nucl. Phys. and Instruments A 608, 19-27 (2009).

E. Kur and A. Zholents, "Investigation of Beam Instability under the Effects of Long-range Transverse Wake Fields in the Berkeley Future Light Source," LBNL Report LBNL10061E (2009). 
AFRD-Zholents, Fawley LB07015 FEL Concepts for Multiple Independent X-ray Beamlines

A. Zholents and G. Penn, "Obtaining two attosecond pulses for x-ray stimulated Raman spectroscopy," LBNL Report LBNL -2534E (2009); to be published in Nucl. Instr. and Meth. A.

ALS-Guo LB07016 Building In-situ Electronic Structure Study Capability with Photonin/Photon-out Soft X-ray Spectroscopy

\section{Refereed Publications}

T. Herranz, X. Deng, A. Cabot, J. Guo, and M. Salmeron; "Influence of the Cobalt Particle Size in the CO Hydrogenation Reaction Studied by In Situ X-Ray Absorption Spectroscopy," J. Phys. Chem. B 113, 10721-10727 (2009).

J. Guo, "Interface Science in Nanoparticles: An Electronic Structure View of Photonin/Photon-out Soft-X-Ray Spectroscopy," Int. J. Quant. Chem. 109, 2714-2721 (2009).

\section{Presentations}

J. Guo, "Photon-in/Photon-out Soft-X-Ray Spectroscopy in Characterization of Solar Nanomaterials," invited talk at 42th Western Regional Meeting 2008, American Chemical Society, Las Vegas, Nevada (September 23-27, 2008).

J. Guo, "Photon-in/Photon-out Soft-X-Ray Spectroscopy in Characterization of Solar Nanomaterials," invited talk at Institute of Physics, Academia Sinica, Taipei, Taiwan (October 3, 2008).

J. Guo, "Photon-in/Photon-out Soft-X-Ray Spectroscopy: An Emerging Probe for Renewable Energy Science," invited talk at The $14^{\text {th }}$ User's Meeting and Workshop, NSRRC, Hsingchu, Taiwan (October 8-9, 2008).

J. Guo, "Electronic Structure Study of Nanostructured Materials from in-situ Photonin/Photon-out Soft-X-Ray Spectroscopy," invited talk at 11-th International Conference on Electronic Spectroscopy and Structure (ICESS-11), Nara, Japan (June 6-11, 2009).

J. Guo, "Photon-in/Photon-out Soft-X-Ray Spectroscopy: An Emerging Probe for Renewable Energy Science," Workshop on Soft X-ray Scattering, National Synchrotron Radiation Research Center, HsinChu, Taiwan (June 12-13, 2009).

J. Guo, "Photon-in/Photon-out Soft-X-Ray Spectroscopy: An Emerging Probe for Renewable Energy Science," invited talk at AVS 56th International Symposium \& Exhibition, San Jose Convention Center, San Jose, California (November 9-13, 2009).

ALS-Hexemer LB08009 Soft X-Ray Scattering as a New Probe of Polymer Systems

Ade et al, "Characterization of Multicomponent Polymer Trilayers with Resonant Soft X-Ray Reflectivity," J. Poly.m Sci. Pol. Phys. 47 (13), 1291-1299 (2009).

Wang et al, "Interfacial Widths of Conjugated Polymer Bilayers," J. Amer. Chem. Soc. 131 (35), 12538+ (2009).

Swaraj et al, "The utility of resonant soft x-ray scattering and reflectivity for the nanoscale characterization of polymers," Eur. Phys. J. Spec. Top. 167, 121-126 (2009). 
ALS-Shapiro et al LB08019 Development of a Laser Goniometer for X-ray and Electron Diffraction Microscopy

Y. Zhao, D. Shapiro, D. McGloin, D. T. Chiu, and S. Marchesini; "Direct observation of the transfer of orbital angular momentum to metal particles from a focused circularly polarized Gaussian beam," Optics Express (in press).

ALS-Yashchuk LB07033 Ultra-high Resolution Optics for Soft X-ray Inelastic Scattering

D. L. Voronov, E. H. Anderson, R. Cambie, J. Meyer-Ilse, E. M. Gullikson, V. V. Yashchuk, H. A. Padmore, M.Ahn, C.-H.Chang, R. K. Heilmann, M. L. Schattenburg; "Ultra high density multilayer sliced grating for EUV and Soft X-rays: Recent Developments," draft for submission to Opt. Eng.

D. L. Voronov, E. H. Anderson, R. Cambie, J. Meyer-Ilse, E. M. Gullikson, V. V. Yashchuk, H. A. Padmore, M. Ahn, C.-H.Chang, R. K. Heilmann, M. L. Schattenburg; "Ultra high density multilayer sliced grating for EUV and Soft X-rays: Recent Developments," Proceedings of SPIE 7448,74480J (2009).

D. L. Voronov, R. Cambie, M. Ahn, E. Anderson, Ch.-H. Chang, E. Gullikson, R. Heilmann, F. Salmassi, M. Schattenburg, V. V. Yashchuk, H. A. Padmore; "Ultra-high resolution optics for EUV and soft x-ray inelastic scattering," Extended Abstract in the Proceedings of The Tenth International Conference on Synchrotron Radiation Instrumentation (Melbourne, Victoria, Australia, September 27 - October 2, 2009).

D. Voronov, E. Anderson, R. Cambie, E. Gullikson, F. Salmassi, T. Warwick, V. V. Yashchuk, H. A. Padmore; "High density dispersive multilayer gratings for soft Xray spectrometers," Extended Abstract in the Proceedings of The Tenth International Conference on Synchrotron Radiation Instrumentation (Melbourne, Victoria, Australia. September 27 - October 2, 2009).

D. L. Voronov, E. H. Anderson, R. Cambie, J. Meyer-Ilse, E. M. Gullikson, V. V. Yashchuk, H. A. Padmore, M.Ahn, C.-H.Chang, R. K. Heilmann, M. L. Schattenburg; "Ultra high density multilayer sliced grating for EUV and Soft X-rays: Recent Developments," Abstract to Optics and Photonics 2009: Optical Engineering and Applications Symposium (San Diego, August $02-06,2009$ ).

Howard A. Padmore, Dmytro L. Voronov, Rossana Cambie, Valeriy V. Yashchuk, Eric M. Gullikson; "Ultra-high density diffraction grating," patent application IB2523P/LBNL043P (October, 2009).

ALS-Yashcuk, Goldberg LB09030 X-Ray Optical Metrology for Coherence-Preserving Adaptive Optics

S. Yuan, M. Church, R. Celestre, G. Y. Morrison, W. R. McKinney, T. Warwick, K. A. Goldberg, V. V. Yashchuk; "Surface slope metrology and interferometric wave front measurements on deformable soft x-ray mirrors performed in the laboratory and in-situ at-wavelength," Extended abstract in the Proceedings of The Tenth International Conference on Synchrotron Radiation Instrumentation (Melbourne, Victoria, Australia. September 27 - October 2, 2009).

S. Yuan, K. Goldberg, V. V. Yashchuk, R. Celestre, T. Warwick, W. R. McKinney, G. Morrison, S. B. Rekawa, I. Mochi, H. A. Padmore; "At-wavelength and optical metrology of bendable x-ray optics for nanofocusing at the ALS," Extended Abstract in the Digest of the OSA Optics and Photonics Congress: Frontiers in Optics 2009, Laser 
Science XXV, Special Symposium on Optics for Imaging at the Nanoscale and Beyond (San Jose, California, USA, October 11-15, 2009).

S. Yuan, V. V. Yashchuk, K. A. Goldberg, R. Celestre, G. Morrison ${ }^{1}$, M. Church, and T. Warwick; "Thermal Effects on Deformable X-ray Mirrors," draft for submission to J. of $X$-ray Optics and Inst.

CH-Bluhm, Wilson LB07009 Chemical Reactions at Liquid/Vapor Interfaces Probed by Photoemission Spectroscopy

E.R. Mysak, D.E. Starr, K.R. Wilson, H. Bluhm; "A combined aerodynamic lens/ambient pressure photoemission spectrometer for the on-stream analysis of aerosol surfaces," accepted for publication in Rev. Sci. Instrum.

CH-Gessner LB08008 Probing Transient Molecular Entanglement Using Femtosecond High Resolution Delayed-Field Coincidence Imaging

O. Kornilov, R. Wilcox, and O. Gessner; "An ultracompact EUV spectrometer and beam profiler in in-situ beam characterization," draft for submission to Rev. Sci. Instrum.

CH-Harris LB09011 Dynamics of Homogeneous Catalysis Reactions Investigated with Transient Two-Dimensional Infrared Spectroscopy on the Pico- to Microsecond Timescale

K. Sawyer, J. Cahoon, J. Shanoski, E. Glascoe, M. Kling, J. Schlegel, M. Zoerb, M. Hapke, J. Hartwig, C. Webster, C. Harris; "Time-resolved IR studies on the mechanism for the functionalization of primary $\mathrm{C}-\mathrm{H}$ bonds by $\mathrm{Cp} * \mathrm{~W}(\mathrm{CO}) 3(\mathrm{Bpin})$, , J. Am. Chem. Soc. (accepted for publication).

CH-Toste LB08021 Metal Nitrosyl Complexes and Catalytic C-H Bond Functionalization

J.M. Schomaker, F.D. Toste, R.G. Bergman; "Cobalt-Mediated [3 + 2]-Annulation Reaction of Alkenes with a,b-Unsaturated Ketones and Imines," Org. Lett. 11, 3698 (2009).

CS-Bell LB07005 Structured, Adaptive Mesh Refinement Method for Multiphase Reactive Transport in Groundwater

G.S.H. Pau, J.B. Bell, K. Pruess, A.S. Almgren, M.J. Lijewski, K. Zhang; "Numerical Studies of Density-driven Flow in $\mathrm{CO}_{2}$ Storage in Saline Aquifers," Proceedings of TOUGH Symposium, September 14-16 2009, Berkeley, California.

G.S.H. Pau, A.S. Almgren, J.B. Bell, M.J. Lijewski, E. Sonnenthal, N. Spycher, T. Xu, G. Zhang; "A Parallel Second-Order Adaptive Mesh Algorithm for Reactive Flow in Geochemical Systems," Proceedings of TOUGH Symposium, September 14-16 2009, Berkeley, California.

G.S.H. Pau, A.S. Almgren, J.B. Bell, and M.J. Lijewski; “Adaptive Mesh Refinement for Incompressible Flow in Porous Media," Phil. Trans. Royal Soc. A 367(1907), 4633-4654 (November, 2009).

D.E.A. van Odyck, J.B. Bell, F. Monmont, N. Nikiforakis; "The Mathematical Structure of Multiphase Thermal Models of Flow in Porous Media," accepted for publication in Proc. Royal Soc. A 465(2102), 523-549 (February, 2009). 
CS-Carter LB08035 Enhancing the Effectiveness of Manycore Chip Technologies for HighEnd Computing

K. Datta, S. Williams, V. Volkov, J. Carter, L. Oliker, J. Shalf, and K. Yelick; "Auto-tuning Stencil Computations on Diverse Multicore Architectures," Scientific Computing with Multicore and Accelerators, Editors J. Dongarra, D. A. Bader, J. Kurzak. (in press).

S. Williams, J. Carter, L. Oliker, J. Shalf, K. Yelick; "Multi-level Auto-tuning of LBMHD on the XT4," Proceedings of the Cray User Group, Atlanta GA, May 2009.

K. Datta, S. Williams, V. Volkov, J. Carter, L. Oliker, J. Shalf, and K. Yelick; "Auto-tuning the 27-pont Stencil Computations for multicore," Proceedings of the $9^{\text {th }}$ International Workshop on Automatic Performance Tuning, Tokyo, Japan, September 2009.

S. Williams, K. Datta, L. Oliker, J. Shalf; "Auto-tuning Memory-Intensive Kernels for Multicore in Performance Tuning of Scientific Applications," Editors D.H. Bailey and R.F. Lucas (in press).

K. Madduri, S. Williams, S. Ethier, L. Oliker, J. Shalf, E. Strohmaier, K. Yelick; "MemoryEfficient Optimization of Gyrokinetic Particle-to-Grid Interpolation for Multicore Processors," Proc. SC2009: High performance computing, networking, and storage conference, Portland, OR, 2009.

CS-Guok, Agarwal LB07007 On-Demand Overlays for Scientific Applications

L. Ramakrishnan, C. Guok, K. Jackson, E. Kissel, D.M. Swany, D. Agarwal; “On-demand Overlay Networks for Large Scientific Data Transfers," accepted for CCGrid 2010.

CS-Rotem, Otoo LB08015 Energy Smart Disk-Based Mass Storage System

E. Otoo, D. Rotem and S.-C. Tsao; "Workload-Adaptive Management of Energy-Smart Disk Storage systems," IASDS09: Workshop on Interfaces and Architectures for Scientific Data Storage, Sept. 2009, New Orleans, Louisiana.

E. Otoo, D. Rotem and S.-C. Tsao; "A new energy saving configuration for RAID Systems," draft for submission to Scientific and Statistical Database Management (SSDBM'10), Heidelberg, Germany.

CS-Shalf LB08002 Holistic Approach to Energy Efficient Computing Architecture

M. Wehner, L. Oliker. J. Shalf; "Green Flash: Designing an energy efficient climate supercomputer," IEEE Spectrum (2009).

G. Hendry, S. Kamil, A. Biberman, J. Chan, B. Lee, M. Mohiyuddin, A. Jain, K. Bergman, L. Carloni, J. Kubiatowicz, L. Oliker, J. Shalf; "Analysis of Photonic Networks for a Chip Multiprocessor Using Scientific Applications," International Symposium on Networks-on-Chip (NOCS) (2009).

M. Mohiyuddin, M. Murphy, S. Williams, L. Oliker, J. Shalf, J. Wawrzynek; "A Case for Hardware/Software Co-Tuning for Power Efficient Scientific Computing," SC09: International Conference for High-Performance Computing, Networking, Storage, and Analysis, Nov. 2009.

S. Kamil, C. Chan, L. Oliker, J. Shalf, S. Williams; "An Auto-Tuning Framework for Parallel Multicore Stencil Computations," accepted for International Parallel \& Distributed Processing Symposium (IPDPS) 2010.

D. Donofrio, L. Oliker. J. Shalf, M. Wehner, C. Rowen, J. Krueger, S. Kamil, M. Mohiyuddin; "Energy-Efficient Computing for Extreme-Scale Science," IEEE Computer (Fall, 2009). 
J. Shalf, M. Wehner, L. Oliker; "The Challenge of Energy-Efficient HPC," SCIDAC Review (Fall, 2009).

S. Kamil, C. Chan, S. Williams, L. Oliker, J. Shalf, M. Howison, E.W. Bethel, Prabhat; "A Generalized Framework for Auto-tuning Stencil Computations," Proceedings of CUG: Cray User Group Conference, Atlanta, GA (2009).

CS-Strohmaier LB08036 Reference Benchmarks for the Dwarfs (Algorithms)

E. Strohmaier; "Generalized Utility Metrics for Supercomputers," International Supercomputing Conference 2009 (ISC'09) and Computer Science - Research and Development, Springer, 23 (3), 185-193 (June, 2009).

K. Madduri, S. Williams, S. Ethier, L. Oliker, J. Shalf, E. Strohmaier, K. Yelick; "MemoryEfficient Optimization of Gyrokinetic Particle-to-Grid Interpolation for Multicore Processors," SC09 International Conference for High-Performance Computing, Networking, Storage, and Analysis (November, 2009).

CS-Wu LB09028 Managing Petascale Data with Emerging Computer Architectures

K. Madduri and K. Wu, "Efficient joins with compressed bitmap indices," Proc. 18th ACM Conf. on Information and Knowledge Management (CIKM 2009) (November, 2009); url: http://dx.doi.org/10.1145/1645953.1646083.

X. Gu, K. Madduri, K. Subramani, H-J. Lai; "Improved Algorithms for detecting negative cost cycles in undirected graphs," Proc. 3rd Int'l. Frontiers of Algorithmics Workshop (FAW 2009), also LNCS 5598, 40-50 (June, 2009); url: http://dx.doi.org/10.1007/978-3642-02270-8 7.

K. Subramani and K. Madduri, "Two-level Heaps: A new priority queue structure with applications to the single source shortest paths problem," Proc. 3rd Int'l. Conf. on Combinatorial Optimization and Applications (COCOA 2009), also LNCS 5573, 186-196 (June, 2009); url: http://dx.doi.org/10.1007/978-3-642-02026-1_17.

L. Gosink, K. Wu, W. Bethel, J.D. Owens, K. Joy; "Data Parallel Bin-Based Indexing for Answering Queries on Multi-Core Architectures," Proc. 21st Int'l. Conf. on Scientific and Statistical Database Management (SSDBM 2009), also LNCS 5566, 110-129. (June, 2009); url: http://dx.doi.org/10.1007/978-3-642-02279-1_9.

K. Madduri, D. Ediger, K. Jiang, D.A. Bader, D. Chavarria-Miranda; “A Faster Parallel Algorithm and Efficient Multithreaded Implementations for Evaluating Betweenness Centrality on Massive Datasets," Proc. 3rd Workshop on Multithreaded Architectures and Applications (MTAAP 2009) (May, 2009); url: http://dx.doi.org/10.1109/IPDPS.2009.5161100.

K. Madduri and D.A. Bader; "Compact Graph Representations and Parallel Connectivity Algorithms for Massive Dynamic Network Analysis," Proc. 23rd IEEE Int'l. Parallel and Distributed Processing Symposium (IPDPS 2009), (May, 2009); url: http://dx.doi.org/10.1109/IPDPS.2009.5161060.

J. Orlin, K. Madduri, K. Subramani, M. Williamson; "A Faster Algorithms for the Single Source Shortest Path Problem with Few Distinct Positive Lengths," Journal of Discrete Algorithms (in press); url: http://dx.doi.org/10.1016/j.jda.2009.03.001. 
ESD-Berryman LB07008 Application of Adjoint Field Methods and Time-Reversal Data Processing to Inverse Problems in Electromagnetics, Seismics, and Ultrasonics

J.G. Berryman and R. Raghavan, "Device, methods, and control for sonic guidance of molecules and other material utilizing time-reversal acoustics," invention, Attorney Docket JIB-2553P, Lawrence Berkeley National Laboratory.

ESD-Collins LB08004 Integrated Earth Systems Climate Modeling and Analysis

W.D. Collins, V. Ramaswamy, A. Conley, M. Iacono; "The significance of shortwave methane forcing for climate change," Eos Trans. AGU 89 (53), Fall Meet. Suppl., Abstract GC51B-01 (2008).

ESD-Jansson, C. LB08033 Maximizing Photosynthetic Yield by Increasing Sink Strength

C. Jansson and T. Northen, "Calcifying cyanobacteria and carbon sequestration," draft invited review for Current Opin. Biotechnol.

C. Jansson, S. Wullschleger, U. Kalluri, G.T. Tuskan; "How can transgenic plants enhance terrestrial carbon sequestration?" draft invited review submitted to Progress in Botany.

E. Mangelsen, D. Wanke, J. Kilian, E. Sundberg, K. Harter, C. Jansson; "Diurnal fluctuations of transcript levels in developing barley caryopses - a microarray-based analysis," submitted to Plant Physiol.

C. Jansson, S. Wullschleger, U. Kalluri, G.T. Tuskan; "Phytosequestration: Carbon biosequestration by plants and the prospects for genetic engineering," invited review accepted for publication in BioScience.

J. Mutisya, C. Sun, C. Jansson; "Circadian oscillation of starch branching enzyme gene expression on the sorghum endosperm," Plant Sign. Behavior. 4, 871-872 (2009).

C. Jansson, A. westerbergh, J. Zhang, A. Hu, C. Sun; "Cassava, a potential biofuel crop in China,". Applied Energy 86, S95-S99 (2009).

ESD-Jansson, J. LB08034 Microbiomics of Complex Microbial Communities in Environmental $\underline{\text { Samples }}$

A. Khoruts, J. Dicksved, J.K. Jansson, M. J. Sadowsky; "Changes in the Composition of the Human Fecal Microbiome Following Bacteriotherapy For Recurrent Clostridium Difficile-Associated Diarrhea," J. of Clinical Gastroenterology (in press).

K. Hjort, M. Bergström, M. Adesina, J.K. Jansson, K. Smalla, S. Sjöling; "Chitinase Genes Revealed and Compared in Bacterial Isolates, DNA Extracts and a Metagenomic Library From a Phytopathogen Suppressive Soil," FEMS Microbiology Ecology (in press), LBNL-2732E.

T.M. Vogel, P.R. Hirsch, P. Siumonet, J.K. Jansson, J.M. Tiedje, J.D. van Elsas, R. Nalin, L. Philippot, M.J. Bailey; "Advantages of the Metagenomic Approach For Soil Exploration: Reply From Vogel et al," Nature Reviews Microbiology (published on line).

J.K. Jansson, B. Willing, M. Lucio, A. Fekete, J. Dicksved, J. Halfvarson, C. Tysk, P. Schmitt-Kopplin; "Metabolomics Reveals Metabolic Biomarkers of Crohn's Disease," PLoS One 4 (7):1-10 (e6386), LBNL-2153.

W. Zhigang, B. Willing, J. Bjerketorp, J.K. Jansson, K. Hjort; "Soft Inertial Microfluidics For High Throughput Separation of Bacteria From Human Blood Cells," Lab on a Chip. 9,1193-1199, LBNL-1539E. 
T.M. Vogel, P. Simonet, J.K. Jansson, P.R. Hirsch, J.M. Tiedje, J.D. van Elsas, M. J. Bailey, R. Nalin L. Philippot; "TerraGenome: A Consortium For The Sequencing of a Soil Metagenome," Nature Reviews Microbiology 7, 1.

M. Unell, P. E. Abraham, M. Shah, B. Zhang, C. Ruckert, N.C. VerBerkmoes, J. Jansson; "Impact of Phenolic Substrate and Growth Temperature on the Arthrobacter Proteome," J. of Proteome Research 8, 1953-1964, LBNL-1565E.

B. Willing, J. Halfvarson, J. Dicksved, M. Rosenquist, G. Jarnerot, L. Engstrand, C. Tysk , J.K. Jansson; "Twin Studies Reveal Specific Imbalances in the Mucosa-Associated Microbiota of Patients With Ileal Crohn's Disease," Inflammatory Bowel Diseases 15, 653-660, LBNL-1222E.

J. Dicksved, M. Lindberg, M. Rosenquist, H. Enroth, J.K. Jansson, L. Engstrand; "Molecular Characterization of the Stomach Microbiota in Patients With Gastric Cancer and Controls," J. of Medical Microbiology 58, 509-516, LBNL-1632E.

N.C. VerBerkmoes, A.L. Russell, M. Shah, A. Godzik, M. Rosenquist, J. Halfvarson, M.G. Lefsrud, J. Apajalahti, C. Tysk, R.L.Hettich, J.K. Jansson; "Shotgun Metaproteomics of the Human Distal Gut Microbiota," The ISME Journal 3, 179-189, LBNL-1134E.

J. Dicksved, L. Zhao, J.K. Jansson; "Human Environment," in Liu, W-T. and J.K. Jansson,. Environmental Molecular Microbiology, 167-190, Caister Academic Press (in press).

B. Willing and J.K. Jansson, The Gut Microbiome - Ecology and function, ASM Press (in press).

ESD-Liu LB09013 Impact of Climate Change on Soil Water Dynamics in Arid Areas

H.H. Liu and K. Zhang, "Impact of Climate Change on Groundwater Recharge in Arid Areas," 2009 AGU Fall Meeting, November 14-18, San Francisco, California.

ESD-Salve LB09023 Identifying and Predicting Climate Change Impacts on the Land-Based Components of the Water Cycle yes 35

W. Dietrich; T. Dawson; R. Salve; K. Simonin; J. Oshun; D. Rempe, I. Fung; "Might rock moisture in shallow fractured bedrock underlying hillslopes provide vegetation resilience to future droughts?" Eos, 90 (52), 29 (December, 2009), Fall Meet. Suppl., Abstract EP52B-08.

R. Salve et al, "Ascendancy of Shallow Bedrock Flow through a Hillslope in Northern California," draft for submission to Water Resources Research.

EETD-Bazjanac LB09003 Embedded Engineering, Construction Materials, and HVAC Components in Modular Energy Systems Simulation

V. Bazjanac, T. Maile, C.M. Rose; "Space boundaries: using original building geometry in building energy performance simulation," draft to be published as an LBNL Report.

V. Bazjanac, "Building energy performance simulation - what is it telling us?" draft for submission to Science.

V. Bazjanac, "Implementation of semi-automated energy performance simulation: building geometry," in Dikbas, A., E. Ergen and H.Giritli (eds.), CIB W78, Proc. 26th conf., Managing IT in Construction. Istanbul, TK 595-602, CRC Press.

Open Geospatial Consortium. 2009. AECOO-1 testbed demonstration, url: http://www.opengeospatial.org/pub/www/aecoo-1/index.html 
EETD-Destaillats LB07014 Understanding the Chemistry of Innovative Air Cleaning Technologies

D. Kibanova, J. Cervini-Silva, H. Destaillats; "Efficiency of clay- $\mathrm{TiO}_{2}$ nanocomposites on the photocatalytic elimination of a model hydrophobic air pollutant," Environmental Science \& Technology 43, 1500-1506 (2009).

N. Quici, M.L. Vera, H. Choi, G. Li Puma, D.D. Dionysiou, M.I. Litter, H. Destaillats; "Effect of key parameters on the photocatalytic oxidation of toluene at low concentrations in air under $254+185 \mathrm{~nm}$ UV irradiation," submitted to Applied Catalysis $B$-Environmental.

D. Kibanova, M.-C. Leroy, M. Sleiman, A. Fernandez-Martinez, L. Charlet, J. Cervini-Silva, $\mathrm{H}$. Destaillats; "Clay- $\mathrm{TiO}_{2}$ nanocomposites for the photocatalytic elimination of indoor pollutants," Proceedings of the $14^{\text {th }}$ international conference on $\mathrm{TiO}_{2}$ photocatalysis ( $\left.\mathrm{TiO}_{2}-14\right)$, Niagara Falls, NY, October 2009.

EETD-Haves LB08037 Development of a Building Operating Platform

F. Balarin, M. D'Angelo, A. Davare, D. Densmore, T. Meyerowitz, R. Passerone, A. Pinto, A. Sangiovanni-Vincentelli, A. Simalatsar, Y. Watanabe, G. Yang, Q. Zhu; "Platformbased design and frameworks: Metropolis and Metro II," in Model-based design of heterogeneous embedded systems; G. Nicolescu, P. Mosterman (Eds.), CRC Press (2009).

A. Pinto, A., M. D'Angelo, C. Fischione, E. Scholte, A. Sangiovanni-Vincentelli; "Synthesis of Embedded Networks for Building Automation and Control," in Proc. of American Control Conference (ACC 08), Seattle, Washington, June 2008.

Y. Yang, A. Pinto, A. Sangiovanni-Vincentelli, P. Haves, M. Wetter; "A Design Flow for Building Automation and Control," draft for submission to IEEE Real-Time Systems Symposium 2010.

P. Nuzzo, A. Pinto, A. Sangiovanni-Vincentelli; "W-BOOM: A Framework for Automatic Management of Wireless Sensor Networks in Building Automation and Control," EECS Department, University of California, Berkeley, Tech. Rep. UCB/EECS-2009-166, Dec. 2009.

EETD-Levine LB07036 China's Energy Future: Changes in Energy Intensity

M. Levine, L. Price, N. Zhou; "The Greening of the Middle Kingdom: The Story of Energy Efficiency in China," The Bridge (National Academy of Engineering) 39 (2) (2009).

EETD-Rubinstein LB08039 Using Internet Protocols (IP) Telephony and Wireless Technologies to Extend the Reach of Conventional Building Automation Systems

J Granderson, F Rubinstein, D DiBartolomeo, G Ghatikar; "The Performance of an Integrated Dimmable Lighting and Motorized Venetian Blind System," draft be submitted to Leukos.

J Granderson, F Rubinstein, D DiBartolomeo, G Ghatikar; "Enhanced Personal Controls for Networked Efficient Lighting Systems," draft for submission to Leukos.

G Ghatikar, D DiBartolomeo, J Granderson, F Rubinstein; "Technology and Communications Convergence for Economics and Scalability of Demand Response," draft for submission to archival journal TBD. 
EETD-Sohn LB08040 Software for Integrated Analysis of Sensor Data for Advanced Energy Controls

M. Najafi, D. Auslander, P. Barlett, P. Haves, M. Sohn; "Modeling and Measurement Constraints in Fault Diagnostics for HVAC systems," submitted to ASME Journal on Controls and Dynamic Systems.

Draft journal manuscript on an approach to proactive fault diagnosis.

EETD-Wetter LB08041 Building Informatics Environment Enabling Rapid Prototyping and Model Extraction for Building Automation Systems

M. Wetter, "Modelica-based Modeling and Simulation to Support Research and Development in Building Energy and Control Systems," J. of Building Performance Simulation 2 (2), 143-161 (2009).

M. Wetter, "The Future of Building Modeling and Simulation," draft to appear in Jan L.M. Hensen and Roberto Lamberts (eds), Building Performance Simulation for Design and Operation, Taylor and Francis Group.

M. Wetter, "Modelica Library for Building Heating, Ventilation and Air-Conditioning Systems," Proc. of the 7th International Modelica Conference, Como, Italy, September 2009.

R. Franke, F. Casella, M. Otter, K. Proelss, M. Sielemann, M. Wetter; "Standardization of thermo-fluid modeling in Modelica.Fluid 1.0," Proc. of the 7th International Modelica Conference, Como, Italy, September 2009.

M. Wetter, "A Modelica-based model library for building energy and control systems," Proc. of the 11th IBPSA Conference, 652-659, Glasgow, Scotland, July 2009.

GN-Pennacchio LB09019 Assessing Epigenomic Approaches for Gene Enhancer Discovery

A. Visel, M.J. Blow, Z. Li, T. Zhang, J.A. Akiyama, A. Holt, I. Plajzer-Frick, M. Shoukry, C. Wright, F. Chen, V. Afzal, B. Ren, E.M. Rubin, L.A. Pennacchio; "ChIP-seq Accurately Predicts Tissue-Specific Activity of Enhancers," Nature 457, 854-8 (12 Feb 2009).

A. Visel, E.M. Rubin, L.A. Pennacchio; "Genomic views of distant-acting enhancers," Nature 461, 199-205 (10 Sep 2009).

M.J. Blow, Z. Li, D.J. McCulley, T. Zhang, J.A. Akiyama, A. Holt, I. Plajzer-Frick, M. Shoukry, C. Wright, F. Chen, V. Afzal, B. Ren, B.L. Black, E.M. Rubin, A. Visel, L.A. Pennacchio; "Genome-Wide Discovery of Evolutionarily Hidden Heart Enhancers," submitted to Nature Genetics.

LSD-Butland LB07010 Functional Interactomics: Integrating Physical and Functional Interaction Networks

M. Babu, G. Musso, J.J. Díaz-Mejía, G. Butland, J.F. Greenblatt, A. Emili; "Systems-level approaches for identifying and analyzing genetic interaction networks in Escherichia coli and extensions to other prokaryotes," Molecular Biosystems 5, 1439-1455 (July 31 2009); url: http://www.rsc.org/publishing/journals/MB/article.asp?doi=b907407d.

J.I. Handford, B. Ize, G. Buchanan, G.P. Butland, J. Greenblatt, A. Emili, T. Palmer; "Conserved Network of Proteins Essential for Bacterial Viability," J. of Bacteriology 191 (15), 4732-4749 (August 2009); url: http://jb.asm.org/cgi/content/full/191/15/4732?view=long\&pmid=19376873 
N. Yeung, B. Gold, G. Butland; "Protein complex formation and FeS cluster cofactor binding properties of E. coli monothiol glutaredoxin GrxD: Scaffold or Sensor?" draft for submission to J. of Biological Chemistry.

M. Babu, N. Yeung, B. Gold, J. Greenblatt, A. Emili, G. Butland; "Genetic and Physical interactions of E. coli monothiol glutaredoxin GrxD reveals potential multiple roles in FeS cluster biosynthesis," draft for submission to Molecular Microbiology or J. of Bacteriology.

LSD-Comolli LB09006 Linking Genomics, Proteomics and Ultrastructural Characterization of Microbial Communities and Their Viruses

L.R. Comolli, B.J. Baker, K.H. Downing, C.E. Siegerist, J.F. Banfield; "Three-dimensional analysis of the structure and ecology of a novel, ultra-small archaeon," ISME J 3(2), 159167 (2009).

B.J. Baker, L.R. Comolli, G.J. Dick, H. Loren, M. Land, N.C. VerBerkmoes, R.L. Hettich, J. F. Banfield; "Enigmatic, ultra-small uncultivated Archaea," submitted to PLOS Biol.

B. Knierim, P. Wilmes, R.I. Webb, K. McDonald, L.R. Comolli, B. Luef, K.H. Downing, J.F. Banfield, P. Hugenholtz, J. Liphardt, M. Auer; "Correlative TEM/FISH Imaging of Microbial Communities," draft for submission to Nature Methods.

LSD-Hang LB08027 Interaction of Fragile X Mental Retardation Protein with Thymine-DNA Glycosylase: Implication in the Molecular Mechanism of Fragile X Syndrome

H. Budworth, P. Wang, A.H. Sarker, D.G. Ciobanu, P.K. Cooper, B. Hang; "Human TDG interactions with FMRP and XPG: a model for their functions in active DNA demethylation," draft for submission to J. of Biological Chemistry.

LSD-Tainer LB09025 Bio Energy Technologies and Science Integrated Efficiently (BETSIE)

K. Hitomi, L. DiTacchio, A.S. Arvai, J. Yamamoto, S.T. Kim, T. Todo, J.A. Tainer, S. Iwai, S. Panda, E.D. Getzoff; "Functional motifs in the (6-4) photolyase crystal structure make a comparative framework for DNA repair photolyases and clock cryptochromes," Proc Natl Acad Sci USA 106 (17), 6962-7 (Apr 28 2009).

G.L. Hura, A.L. Menon, M. Hammel, R.P. Rambo, F.L. Poole 2nd, S.E. Tsutakawa, F.E. Jenney Jr, S. Classen, K.A. Frankel, R.C. Hopkins, S.J. Yang, J.W. Scott, B.D. Dillard, M.W. Adams, J.A. Tainer; "Robust, high-throughput solution structural analyses by small angle X-ray scattering (SAXS)," Nat. Methods 6 (8), 606-12. (2009 Aug., Epub 2009 Jul 20).

LSD-Wyrobek LB06035 Expression Profiling of Radiation and Cancer Susceptibility Genes

T. Straume, S.A. Amundson, W.F. Blakely, F.J. Burns, A. Chen, N. Dainiak, S. Franklin, J.A. Leary, D.J. Loftus, W.F. Morgan, T.C. Pellmar, V. Stolc, K.W. Turteltaub, A.T. Vaughan, S. Vijayakumar, A.J. Wyrobek; "Meeting Report ---NASA Radiation Biomarker Workshop," Radiation Research 170 (3), 393-405 (Sept. 2008).

X.R. Lowe, S. Bhattacharya, F. Marchetti, A.J. Wyrobek; "Early brain response to low-dose radiation exposure involves molecular networks and pathways associated with cognitive functions, advanced aging, and Alzheimer's disease," Radiation Research 171 (1), 53-65 (Jan. 2009). 
X.R. Lowe, F. Marchetti, X. Lu, A.J. Wyrobek; "Molecular stress response in the CNS of mice after systemic exposure to interferon-alpha, ionizing radiation and ketamine," Neurotoxicology 30 (2), 261-8 (Mar. 2009).

J.C. Garbe, S. Bhattacharya, B. Merchant, E. Bassett, K. Swisshelm, H.S. Feiler, A.J. Wyrobek, M.R. Stampfer; "Molecular distinctions between the stasis and telomere attrition senescence barriers demonstrated by long-term culture of normal human mammary epithelial cells," Cancer Research 69 (19), 7557-68 (Oct. 12009 ).

MSD-Jérôme LB08029 Transport in Thin Polymer Films

M. Mezger, B. Jérôme, J.B. Kortright, M. Valvidares, E.M. Gullikson, S. Nannarone, A. Giglia, N. Mahne: "Orientation of side chain liquid crystalline polymer thin films studied by resonant soft x-ray reflectivity," ACS Sping Meeting 2010, March 2010, to be presented.

M. Mezger, B. Jérôme, J.B. Kortright, M. Valvidares, E.M. Gullikson, S. Nannarone, A. Giglia, N. Mahne: "Orientation of side chain liquid crystalline polymer thin films studied by resonant soft x-ray reflectivity," draft paper.

MSD-Long LB08030 Conducting Metal-Organic Frameworks

J.N. Behera, D.M. D'Alessandro, N. Soheilnia, J.R. Long; "Synthesis and Characterization of Ruthenium and Iron-Ruthenium Prussian Blue Analogues," Chem. Mater. 21, 1922-1926 (2009).

MSD-Milliron LB09017 Solution-Processed Inorganic Composites with Mixed Transport Characteristics

R. Tangirala, J.L. Baker, A.P. Alivisatos, D.J. Milliron; "Modular inorganic nanocomposites by conversion of nanocrystal superlattices," submitted to Angewandte Chemie.

MSD-Siddiqi LB07040 Quantifying the Quantum Backaction of a Non-Linear Dispersive Measurement

D. Slichter, O. Naaman, I. Siddiqi; "Millikelvin thermal and electrical performance of lossy transmission line filters," Applied Physics Letters 94, 192508 (15 May 2009); url: http://scitation.aip.org/getabs/servlet/GetabsServlet?prog=normal\&id=APPLAB0000940 $00019192508000001 \&$ idtype $=$ cvips\& gifs $=$ yes\&ref $=$ no.

\section{MSD-Smit, Xu LB09029 Self-assembly of Membrane Proteins}

J.M. Rodgers, M. Webb, B. Smit; "Alcohol solubility in a lipid bilayer -- Efficient grandcanonical simulation of an interfacially-active molecule," submitted to J. of Chemical Physics.

F. de Meyer, A. Benjamini, J.M. Rodgers, Y. Misteli, B. Smit; "Molecular simulation of the DMPC-cholesterol temperature-composition structure diagram," draft for submission to Biophysical Journal. 
MSD-Urban LB08044 Understanding How Nanoscale Interfaces Modify Predicted Optical, Vibrational, and Electronic Properties

A.M. Schwartzberg, S. Aloni, T. Kuykendall, P.J. Schuck, J.J. Urban; "Directional Nanoscale White-Light Generation by Efficient Multiphoton Excitation in Gallium Nitride Nanowires," submitted to Nature Nanotechnology.

M. Schmidt, A.M. Schwartzberg, P.N. Perera, A. Weber-Bargioni, A. Carroll, P. Sarkar, E. Bosneaga, J.J. Urban, J. Song, M.Y. Balakshin, E.A. Capanema, M. Auer, P.D. Adams, V.L. Chiang, P.J. Schuck; "Label-free in situ imaging of lignification in the cell wall of low lignin transgenic," Populus trichocarpa," Planta. (in press).

MSD-Wu LB09027 Microscopic Mechanism of Resistance Switching Memory Effect

J. Cao, E. Ertekin, V. Srinivasan, W. Fan, S. Huang, H. Zheng, J.W.L. Yim, D.R. Khanal, D.F. Ogletree, J.C. Grossman, J. Wu; "Strain Engineering and One-Dimensional Organization of Metal-Insulator Domains in Single-Crystal VO2 Beams," Nature Nanotech. 4, 732 (2009, cover highlight); url: http://www.mse.berkeley.edu/ jwu/publications/Cao-NatureNano-09.pdf

J. Cao, W. Fan, H. Zheng, J. Wu; "Thermoelectric Effect across the Metal-Insulator Domain Walls in VO2 Microbeams," Nano Lett. (in press); url: http://www.mse.berkeley.edu/ jwu/publications/Cao-NanoLett-09.pdf

W. Fan, S. Huang, J. Cao, E. Ertekin, C. Barrett, D.R. Khanal, J.C. Grossman, J. Wu; "Superelastic Metal-Insulator Phase Transition in Single-Crystal Vanadium Dioxide Nanobeams," Phys. Rev. B, Rapid Commun. (in press).

J. Cao, W. Fan, K. Chen, N. Tamura, M. Kunz, J. Wu; "Constant threshold resistivity in the metal-insulator transition of VO2: evidence of Mott transition," submitted to Phys. Rev. Lett.

J. Cao, W. Fan, D.F. Ogletree, K. Chen, N. Tamura, M. Kunz, C. Barrett, J. Seidel, E. Saiz, J. $\mathrm{Wu}$; "Mapping and Exploration of Extensive Stress-Temperature Phase Diagram of Vanadium Dioxide," draft for submission to Nature Materials.

NSD-Basunia, Clark LB07012 New Experimental Initiative to Deduce (n,f) Crossection for Advance Fuel Cycle Studies

M.S. Basunia, R.M. Clark, B.L. Goldblum, L.A. Bernstein, L. Phair, J.T. Burke, C.W. Beausang, D.L. Bleuel, B. Darakchieva, F.S. Dietrich, M. Evtimova, P. Fallon, J. Gibelin, R. Hatarik, C.C. Jewett, S.R. Lesher, M.A. McMahan, E. Rodriguez-Vieitez, M. Wiedeking; "The $\left({ }^{3} \mathrm{He}, \mathrm{t} f\right)$ as a surrogate reaction to determine $(n, f)$ cross sections in the 10 to $20 \mathrm{MeV}$ energy range," Nucl. Instr. and Meth. in Phys. Res. B 267, 1899-1903 (2009).

S.R. Lesher, J.T. Burke, L.A. Bernstein, H. Ai, C.W. Beausang, D.L. Bleuel, R.M. Clark, F.S. Dietrich, J.E. Escher, P. Fallon, J. Gibelin, B.F. Goldblum, I.Y. Lee, A.O. Macchiavelli, M.A. McMahan, K.J. Moody, E.B. Norman, L. Phair, E. RodriguezVieitez, N.D. Scielzo, M. Wiedeking; "Surrogate ratio method in the actinide region using the ( $\alpha$, a'f) Reaction," Phys. Rev. C 79, 044609 (2009).

N.D. Scielzo, J.E. Escher, J.M. Allmond, M.S. Basunia, C.W. Beausang, L.A. Bernstein, D.L. Bleuel, J.T. Burke, R.M. Clark, F.S. Dietrich, P. Fallon, J. Gibelin, B.L. Goldblum, S.R. Lesher, A.O. Macchiavelli, M.A. McMahan, E.B. Norman, L. Phair, E. RodriquezVieitez, S.A. Sheets, I.J. Thompson, M. Wiedeking; "Measurement of gamma-emission 
branching ratios for ${ }^{154,156,158} \mathrm{Gd}$ compound nuclei: tests of surrogate nuclear reaction approximations for (n,gamma) cross sections," draft for submission to Phys. Rev. C.

NSD-Klein LB08011 Development of a $100 \mathrm{~km} 3$ Neutrino Detector for Ultra High Energy Neutrinos

E. Couderc and S. Klein, "Coherent rho^ 0 photoproduction in bulk matter at high energies," Phys. Rev. Lett. 103, 062504 (Aug. 6, 2009).

A popular writeup of the above article appeared in Physical Review Focus:

http://focus.aps.org/story/v24/st6

NSD-Lesko LB07020 Physics Detector and Sensor Technologies Applied to Geological and Geophysical Applications at DUSEL

Conference Proceedings

M. Amman, P. Luke, Y. Chan, K. Lesko; "Segmented P-Type Point Contact Germanium Detector," IEEE Conference, Orlando, Florida, Oct 20-30, 2009.

P. Luke, C. Tindall, K. Vetter, Y. Chan, K. Lesko; "Low-Noise Low-Mass Front-End Electronics for Low-Background Experiments Using Germanium Detectors," IEEE

Conference, Orlando, Florida, Oct 20-30, 2009.

\section{Internal Reports}

Y. Chan et al, "P-Type Point Contact Detector Development," [M-TECHDOCDET-2009055] unpublished.

Y. Chan et al, "Low-Mass Front-End (LMFE) Development," [M-TECHDOCDET-2009057] unpublished.

Y. Chan et al, "PPC Single Interaction Site Analysis - the Amman Method," [MTECHDOCDET-2009-063] unpublished.

M. Amman et al, "Segmented P-type Point Contact Germanium Detector," [MTECHDOCDET-2009-056] unpublished.

Y. Chan et al, "Waveform Library for the LBNL PPC1 Detector," [M-TECHDOCDET2009-062] unpublished.

Y. Chan et al, "Segment-Signal and Single Site Event Study for a Segmented PPC Detector," [M-TECHDOCDET-2009-061] unpublished.

P. Dobson and R. Salve, "Preliminary Investigation into Conducting Geological $\mathrm{CO}_{2}$ Sequestration Studies at the DUSEL Facility, Homestake Mine, South Dakota," LBNL report (Nov. 2009).

NSD-Yuan LB09031 Theoretical Study of Nucleon Structure

F. Yuan, J. Zhou; "Collins Function and the Single Transverse Spin Asymmetry," Phys. Rev. Lett. 103, 052001 (2009).

W. Vogelsang, F. Yuan; "Next-to-leading Order Calculation of the Single Transverse Spin Asymmetry in the Drell-Yan Process," Phys. Rev. D 79, 094010 (2009).

C. Marquet, B. Xiao, F. Yuan; "Semi-inclusive Deep Inelastic Scattering at Small-x," Phys. Lett. B 682, 207 (2009).

J. Zhou, F. Yuan, Z. Liang: "Drell-Yan Lepton Pair Azimuthal Asymmetry in Hadronic Processes,” Phys. Lett. B 678, 264 (2009). 
J. Zhou, F. Yuan, Z. Liang; "QCD Evolution of the Transverse Momentum Dependent Correlations," Phys. Rev. D 79, 114022 (2009).

J. Zhou, F. Yuan, Z. Liang; "Transverse momentum dependent quark distributions and polarized Drell-Yan processes," ], to appear in Phys. Rev. D, LBNL-2612E, e-Print: arXiv:0909.2238 [hep-ph.

PBD-Crooks LB08005 Understanding the Nanothermodynamics of Molecular Machines

E. H. Feng and G.E. Crooks, "Far-From-Equilibrium Measurements Of Thermodynamic Length," Phys. Rev. E 79, 012104 (2009); 10.1103/PhysRevE.79.012104.

G.E. Crooks, "Comment Regarding 'On The Crooks Fluctuation Theorem And The Jarzynski Equality,' [J. Chem. Phys. 129, 091101 (2008)] and 'Nonequilibrium FluctuationDissipation Theorem Of Brownian Dynamics' [J. Chem. Phys. 129, 144113 (2008)], ' J. Chem. Phys. 130, 107101 (2009); doi:10.1063/1.3080751.

D. Greenfield, A.L. McEvoy, H. Shroff, G.E. Crooks, N.S. Wingreen, E. Betzig, J. Liphardt; "Self-Organization Of The Escherichia Coli Chemotaxis Network Imaged With SuperResolution Light Microscopy," Plos Biol. 7:e1000137 (2009); doi:10.1371/journal.pbio.1000137.

D.A. Sivak and G.E. Crooks, "Near-equilibrium Measurements of Non-Equilibrium Free Energy," submitted.

PBD-Fletcher LB09008 Engineering Environmental Sensitivity in an Artificial Cell

J. Stachowiak, D. Richmond, T. Li, F. Brochard-Wyart, and D.A. Fletcher; "Inkjet printing of unilamellar lipid vesicles for cell-like encapsulation," Lab on a Chip 9 (14), 2003-9 (2009, cover image).

PBD-Groves LB09010 Applications of Hybrid Live Cell - Synthetic Devices for Cancer Research

K. Salaita, P.M. Nair, R.S. Petit, R.M. Neve, D. Das, J.W. Gray, J.T. Groves; "Physical Manipulation of EphA2 Spatial Organization Alters Cellular Response to Ephrin-A1," submitted to Science.

PBD-Liphardt LB08013 Light-boosted Fermentation in the Yeast Saccharomyces cerevisiae

J. Walter, J. Triffo, M. Auer, J. Liphardt; "Design and Synthesis of Artificial Chloroplasts in S. Cerevisiae," draft.

J. Walter, D. Greenfield, J. Liphardt; "Potential of light-harvesting proton pumps for bioenergy," Current Opinion in Biotechnology, "Energy Biotech" special focus edition (in press).

PD-Battaglia LB08028 Advanced Silicon Detectors for Future Short Pulse X-ray Sources

M. Battaglia, D. Bisello, D. Contarato, P. Denes, P. Giubilato, L. Glesener, S. Mattiazzo, C.Q. Vu; " Monolithic Pixel Sensors in Deep-Submicron SOI Technology," in JINST 4, P04007 (Mar. 2009) and e-Print: arXiv:0903.3205.

M. Battaglia, D. Bisello, D. Contarato, P. Denes, P. Giubilato, L. Glesener, S. Mattiazzo, C. $\mathrm{Vu}$; "Monolithic Pixel Sensors in Deep-Submicron SOI Technology with Analog and Digital Pixels," Nucl. Instrum. Meth. A 604, 380-384 (June 2009) and e-Print: arXiv:0811.4540 [physics.ins-det]. 
S. Mattiazzo, M. Battaglia, D. Bisello, D. Contarato, P. Denes, P. Giubilato, D. Pantano, N. Pozzobon, M. Tessaro, J. Wyss; "Total dose effect on a FD-SOI technology for monolithic pixel sensors," to appear in the Proceedings of the RADECS 2009 Conference.

PD-Bauer LB07004 Soft Collider Effective Theories Applied to Collider Physics

A. Hornig, C. Lee, G. Ovanesyan; "Infrared Safety in Factorized Hard Scattering CrossSections," Phys. Lett. B 677, 272-277 (2009).

C.W. Bauer, B.O. Lange; "Scale setting and resummation of logarithms in pp ---> V + jets," e-Print: arXiv:0905.4739 [hep-ph].

A. Hornig, C. Lee, G. Ovanesyan; "Effective Predictions of Event Shapes: Factorized, Resummed, and Gapped Angularity Distributions," JHEP 0905, 122 (2009).

S.D. Ellis, A. Hornig, C. Lee, C.K. Vermilion, J.R. Walsh; "Consistent Factorization of Jet Observables in Exclusive Multijet Cross-Sections," e-Print: arXiv:0912.0262 [hep-ph].

C.W. Bauer, F.J. Tackmann, J. Thaler; "The NLO Cascade: Improving arbitrary NLO calculations with leading-logarithmic resummation," draft.

PD-Gilchriese, Haber LB08048 Development of Multi-Modular Assemblies with Reduced Material and Services for Tracking at Future Colliders

M. Cepeda, S. Dardin, M. Gilchriese, W.K. Miller, W.O. Miller" R. Post; "Pixel Detector Local Mechanical Supports with Integrated Cooling Based on Thermally Conducting Carbon Foam," ATLAS Internal Note.

PD-Padmanabhan, White LB08046 Calibrating Baryon Acoustic Oscillations for Future Dark Energy Experiments

X. Xu, M. White, N. Padmanabhan, D.J. Eisenstein, J. Eckel, K. Mehta, M. Metchnik, P. Pinto, H.-J. Seo; "A new statistic for analyzing baryon acoustic oscillations," draft.

A. Slosar, S. Ho, M. White, T. Louis; "The acoustic peak in the Lyman alpha forest," JCAP 10, 19 (Oct. 2009).

H.-J. Seo, J. Eckel, D.J. Eisenstein, K. Mehta, M. Metchnik, N. Padmanabhan, P. Pinto, R. Takahashi, M. White, X. Xu; "High-precision predictions for the acoustic scale in the non-linear regime," arXiv:0910.5005 (Oct. 2009).

N. Padmanabhan and M. White, "Calibrating the baryon oscillation ruler for matter and halos," Phys. Rev. D 80, 063508 (Sept. 2009).

Y. Noh, M. White, N. Padmanabhan; "Reconstructing baryon oscillations," arXiv:0909.1802 (Sept. 2009).

J. Carlson, M. White, N. Padmanabhan; "Critical look at cosmological perturbation theory techniques," Phys. Rev. D 80, 043531 (Aug. 2009).

M. White and N. Padmanabhan, "Breaking halo occupation degeneracies with marked statistics," MNRAS 395, 2381 (June 2009).

N. Padmanabhan, M. White, J.D. Cohn; "Reconstructing baryon oscillations: A Lagrangian theory perspective," Phys. Rev. D 79, 063523 (Mar. 2009).

H.-J. Seo, E.R. Siegel, D.J. Eisenstein, M. White; "Nonlinear Structure Formation and the Acoustic Scale," ApJ 686, 13 (Oct. 2008).

N. Padmanabhan and M. White, "Constraining anisotropic baryon oscillations," Phys. Rev. D 77, 123540 (June 2008). 
PD-Schlegel LB07025 Baryon Oscillations and Dark Energy: Protyping Instruments

D.J. Schlegel et al, "BigBOSS BigBOSS: The Ground-Based Stage IV BAO Experiment," submitted to Astro2010, the National Research Council's Astronomy and AstrophysicsDecadal Survey, April 1, 2009; url: http://xxx.lanl.gov/abs/0904.0468

\section{PD-Smoot, Leauthaud LB08020 Decoding Dark Energy with Weak Gravitational Lensing}

\section{Refereed Publications}

Leauthaud et al, "A Weak Lensing Study of X-ray Groups in the COSMOS survey: Form and Evolution of the Mass-Luminosity Relation," ApJ (in press), arXiv:0910.5219.

Rhodes et al, "The effects of Charge Transfer Inefficiency (CTI) on galaxy shape measurements," submitted to ApJ.

Massey et al, "Pixel-based correction for Charge Transfer Inefficiency in the Hubble Space Telescope Advanced Camera for Surveys," MNRAS (in press), arXiv:0909.0507.

Drory et al, "The Bimodal Galaxy Stellar Mass Function in the COSMOS Survey to z 1: A Steep Faint End and a New Galaxy Dichotomy," ApJ (in press), arXiv:0910.5720.

Faure et al, "On the Contribution of Large-Scale Structure to Strong Gravitational Lensing," ApJ 695 (2), 1233-1243 (2009).

\section{Talks and Seminars}

S. Perlmutter, D. Schlegel, A. Leauthaud; “The Hidden side of our Universe," Public Lecture, Science at the Theater series, October 2009.

A. Leauthaud, "Weak Gravitational Lensing with COSMOS: Form and Evolution of the Mass-Luminosity, Relation," Colloquium at UC Davis, Oct 2009.

A. Leauthaud, "Weak Gravitational Lensing with COSMOS: Form and Evolution of the Mass-Luminosity Relation,” Santa Fe Cosmology Workshop - Santa Fe - July 2009.

A. Leauthaud and R. Nakajima, "What is Gravitational Lensing?" LBNL Summer Lecture Series- July 2009.

Cross-Div.-Auer et al LB08047 Integrated Tools in Multiscale Imaging

B. Knierim, L.R. Comolli, B. Luef, R.I. Webb, M. Auer, J.F. Banfield, P. Wilmes; "Concomitant high-resolution phylogenetic identification and ultrastructural characterization of mixed microbial consortia," draft for submission to Nature Methods.

B. Knierim, R. Webb, B. van Leer, B. Lich, T. Goddard, K. McDonald, M. Auer; "Highresolution 3D community organization of the lignocellulose-degrading termite hindgut as revealed by FIB/SEM," draft for submission to J. of Structural Biology.

D. Greenfield, A.L. McEvoy, H. Shroff, G.E. Crooks, N.S. Wingreen, E. Betzig, J. Liphardt; "Self-Organization of the Escherichia coli Chemotaxis Network Imaged with SuperResolution Light Microscopy," PLoS Biology 7 (6), e1000137 (2009, Science Editors' Choice commentary and Nature Chemical Biology Research Highlight).

A.R. Lowe, J.J. Siegel, P. Kalab, M. Siu, K. Weis, J. Liphardt; "Selectivity Mechanism of the Nuclear Pore Complex Characterized by Single Cargo Tracking," submitted to Nature. 
Cross-Div.-Lee, S.-W. LB06031 Fabrication of Photovoltaic Devices Using Nano-structured Biomaterials

A. Merzlyak and S.-W. Lee, "Construction Rules of Major Coat Engineered M13 Phage," Bioconjugate Chemistry (in press).

A. Merzlyak, S. Indrakanti, S.-W. Lee; "Genetically Engineered Nanofiber-Like Viruses For Tissue Regenerating Materials," Nano Lett. 9, 856-852 (2009).

W.J. Chung, M. Sena, S.-W. Lee; "Hierarchical organization of the phage for photonic band gap materials," draft for submission to Nature Materials.

W.J. Chung, E.-S. Park, C. Zueger, M. Sena, S.-W. Lee; "Piezoelectric energy conversions of the hierarchically ordered phage films," draft for submission to Nature Materials.

Cross-Div.-Schroeder et al LB08018 Coupled Process Models, Separations, and Monitoring for Advanced Nuclear Fuel Cycles

R. K. Crockett, P. Colella, D. T. Graves; “A Cartesian Grid Embedded Boundary Method for Solving the Poisson and Heat Equations with Discontinuous Coefficients in Three Dimensions," submitted to J. of Computational Physics.

J. Bickel, T. Cisneros, M. Laufer, L. Li, P.F. Peterson; "Conceptual Design, Experiments, and Analysis for the Core of an FHR Test Reactor," submitted to the International Congress on Advances in Nuclear Power Plants (ICAPP), San Diego, CA 2010.

S.G. Minasian, J.L. Krinsky, J.D. Rinehart, V.A. Williams, R. Copping, T. Tyliszczak, M. Janousch, D.K. Shuh, J. Arnold; “A Comparison of 4f vs. 5f Metal-Metal Bonds in $\left(\mathrm{CpSiMe}_{3}\right)_{3} \mathrm{M}-\mathrm{ECp} *\left(\mathrm{M}=\mathrm{Nd}, \mathrm{U} ; \mathrm{E}=\mathrm{Al}, \mathrm{Ga} ; \mathrm{Cp}^{*}=\mathrm{C}_{5} \mathrm{Me}_{5}\right)$ : Synthesis, Thermodynamics, Magnetism, and Electronic Structure,” J. Amer. Chem. Soc. 38, 13767-13783 (2009).

D.E. Bugaris, J.A. Ibers, R. Copping, T. Tyliszczak, D.K. Shuh; " $\mathrm{La}_{2} \mathrm{U}_{2} \mathrm{Se}_{9}$ : An Ordered Lanthanide/Actinide Chalcogenide with a Novel Structure Type," for submission to Inorganic Chemistry.

B. Jeon, N. Gronbech-Jensen, R. Copping, A. Canning, D.K. Shuh; "Coordination Characteristics of Uranyl BBP Complexes: Electronic Structure Analysis," draft.

B. Jeon, N. Gronbech-Jensen, R. Copping, A. Canning, D.K. Shuh; "Electronic Structure Calculations of Ce/Pu-maltol Ligand Complexes," draft.

R. Copping, B. Jeon, S. J. Teat, M. Janousch, T. Tyliszczak, A. Canning, D.K. Shuh; "Structure and Bonding in Uranyl 2,6-Bis(2-benzimidazyl)pyridine Complexes," draft for submission to Inorganic Chemistry.

R. Copping, S.J. Teat, D.K. Shuh; "A Mixed Valence Uranium Trimer," draft for submission to Chem. Commun.

R. Copping, S.J. Teat, M. Janousch, T. Tyliszczak, D.K. Shuh; "Scanning Transmission Xray Microscopy of Homoleptic Trivalent f-element N-donor Complexes," draft for submission to Inorganic Chemistry.

B. Ludewigt, R. Crabbs, B. Quiter; "Study of Nuclear Resonance Fluorescence for Fuel Cycle Material Assay," final report for the AFCI Safeguards Campaign, WP number: LB0915040322 (Oct. 2009).

B. Ludewigt, D.H. Chivers, B. Goldblum, K. Alfonso, R. Crabbs, J. Lorenzo, Y. Wu; "Simulations in Support of Experimental Design," final report for AFCI Safeguards Campaign, WP number: LB0915040323 (Sept. 2009). 